\title{
CRT LIGHT-HEAD \\ FOR AN \\ AUTOMATIC DRAFTING SYSTEM
}

A Thesis

Submitted to the Faculty of Graduate Studies and Research

in Partial Fulfilment of the Requirements

For the Degree of

Master of Science

in the

Department of Electrical Engineering

University of Saskatchewan

by

Kee Shiu Lee

Saskatoon, Saskatchewan

(c) $1976, \mathrm{~K} . \mathrm{S} . \mathrm{LEE}$ 


\section{COPYRIGHT}

The author has agreed that the Library, University of Saskatchewan, may make this thesis freely available for inspection. Moreover, the author has agreed that permission for extensive copying of this thesis for scholarly purposes may be granted by the professor or professors who supervised the thesis work recorded herein or, in their absence, by the Head of the Department or the Dean of the College in which the thesis work was done. It is understood that due recognition will be given to the author of this thesis and to the University of Saskatchewan in any use of the material in this thesis. Copying or publication or any other use of the thesis for financial gain without approval by the University of Saskatchewan and the author's written permission is prohibited.

Requests for permission to copy or to make any other use of material in this thesis in whole or in part should be addressed to:

Head of the Department of Electrical Engineering University of Saskatchewan

Saskatoon, Saskatchewan Canada 


\section{ACKNOWLEDGEMENTS}

The author wishes to express his sincere thanks to Dr. A.R. Boyle for his advice and encouragement during both the course of this project and the preparation of this thesis.

Appreciation is also extended to all people in the Graphic Systems Design and Application Group, Department of Electrical Engineering, University of Saskatchewan. In particular, the author would like to thank Mr. W.T. Wilkie whose comments and suggestions clarified several points.

The financial support provided by NRC grant No. A3622 is gratefully acknowledged. 
UNIVERSITY OF SASKATCHEWAN

ELECTRICAL ENGINEERING ABSTRACT 76 A176

- "CRT LIGHT-HEAD FOR AN AUTOMATIC DRAFTING SYSTEM"

Student: Kee-Shiu Lee

Supervisor: A.R. Boyle

M.Sc. Thesis presented to the College of Graduate Studies

\section{$\underline{\text { ABSTRACT }}$}

This thesis describes the method of implementation of a cathode-ray tube (CRT) for use with a high quality precision automatic drafting system intended for the preparation of maps on a flatbed drafting table. A CRT light-head, mounted in place of the normal optical-mechanical light-head on a drafting table, enables the production of a wide range of symbolized line features as well as symbols and names. The image on the CRT, which is generated according to the information received from a library file in the computer, is projected via an optical system onto a photo-sensitive material. This system also permits the application of 'area-fill' by means of the CRT light-head to save the use of 'peel-coat.'

The system utilizes a PDP8/e mini-computer with $8 \mathrm{~K}$ words of core memory, an $1.6 \mathrm{~N}$ word disc unit, a 9-track magnetic tape unit, a CRT light-head, and a precision flatbed drafting table. 
COPYRIGHT

ACKNOWLEDGENENTS

ABSTRACT

TABLE OF CONTENTS

LIST OF FIGURES

vii

LIST OF FLOWCHARTS

1. INTRODUCTION 1

1.1 General 1

1.2 Disadvantage of an opto-mechanical 3

1.3 Previous work on a CRT light-head 4

1.4 Objectives of the present work ?

2. SYSTEM REQUIREMENTS AND COMPONENTS SELECTION 9

2.1 General

2.2. Requirements of the system 10

2.3 Selection of system components 15

2.3.1 Optical lens 15

2.3.2 Photographic film 17

2.3.3 Drafting table 20

2.3.4 CRT unit and its assoeiated 22 electronics

3. LINE DRAFTING 25

3.1 General 25

3.2 System block diagram 26

3.3 Line generator and its control 29 
3.3.1 Rotation control

3.3.2 Width control

3.3.3 Intensity control 42

3.4 The input/output transfer instructions 47

3.5 Sequence of operation 47

3.6 Samples of line drafting $\quad 50$

3.6.1 General 50

3.6.2 Drafting on film 52

3.6.2.1 Exposure test 52

3.6.2.2 'Mathematically based' $\quad 55$

3.6.2.3 Digitized cartographic 58

3.6.3 Causes of irregularities $\quad 60$

4. AREA FILI 61

4.1 General 61

4.2 Programming 62

4.2.1 Input parameters 62

4.2.1.1 Window 62

4.2.1.2 Fill-in pattern code 62

4.2.1.3 Data format 64

4.2.2 Processing a filling-line 64

4.2.2.1 Intersection point 65

4.2.2.2 Point-in detection $\quad 66$

4.2.3 Example of area fill 67

4.2.4 Application 69

$\begin{array}{ll}4.3 \text { Samples of work } & 71\end{array}$

5. CHARACTER PRINTING 80

5.1 General $\quad 80$

5.2 Programming $\quad 80$

5.3 Advances on previous work 82

5.4 Samples of present work 85 
Page

6. CONCLUSIONS AND RECOMMENDATIONS 91

6.1 Conclusions 91

6.2 Recommendations 93

REFERENCES 96

$\begin{array}{ll}\text { APPENDICES } & 98\end{array}$

Appendix A Automatic drafting $\quad 98$

Appendix B Forward-look linear interpolation 108

Appendix C Characteristics of a rectangular 118 light spot

Appendix D Schematic diagrams of the 125 previous character generation unit

Appendix $E$ Schematic diagrams of the complete system 


\section{LIST OF FIGURES}

Figure

Page

1.1 Block diagram of an automatic drafting system

1.2 Block diagram of CRT photo-typesetter

2.1 Cartographic line

2.2 The $\mathrm{D} \log \mathrm{E}$ curve

2.3 H and D curves for type 10 Kalvar

3.1 Block diagram of interfaces and control for CRT light-head

3.2 CRT light-head

3.3a CRT light-head mounted on Gerber 22 table

3.3c Interface unit of Gerber 22 table

3.3d Interface unit of the CRT light-head

3.4 Block diagram of the line generation unit

3.5 Integrator

3.6a Top view of the drafting table

3.6b Front view of the CRT screen

3.7 Block diagram of the width control unit

3.8 Waveform of the signals from the width control unit

40

3.9 Block diagram of the voltage divider

3.10 Block diagram of intensity control

3.11 I.0.T. for the system

48

3F.1 Sample of exposure test 
Figure

Page

3F.2 Sine-wave $($ width $=0.3 \mathrm{~mm}) \quad 56$

$3 \mathrm{~F} .3 \quad$ Sine-wave $($ width $=0.5 \mathrm{~mm}) \quad 57$

3F.4 Sample of shoreline 59

4.1a Fill-in parameters 63

4.1b Mid-point approaching 63

4.2 Example of area-fill 68

4.3 Boundary of area-fill (611 display) 73

4.4 Sample of block-fill 74

4.5 Sample of area-fill by varying the 75

4.6 Six fill-in pattern codes 76

4.7 Sample of area separation 77

4F.1a Boundary of area 78

4F.1b Sample of area-fill 78

5.1 Sample of character printing (611 display) 86

$5.2 \quad$ Sample of scaling $\quad 87$

5.3 Sample of rotation (area-fill technique) 88

5.4 Sample of rotation (scan-line format) 89

5F.1 Sample of character printing 90

A1 Relative exposure across a straight line 102

A2 Relative exposure across a curved Iine 103

B1 Quantised coastline on $0.1 \mathrm{~mm}$ matrix 111

B2 Iine regeneration by point-to-point 111

B3 4-point and 8-point look-ahead line 112 regeneration. 
Figure

Page

BF.1 Iine regeneration by point linear

interpolation

BF.2 Line regeneration by 4-point lookahead linear interpolation

BF.3 Line regeneration by 8-point look-

C1 Rectangular light spot

C2 Relative exposure varies with the width

121 of the rectangular light spot

C3 Relative exposure varies with the radius of curvature

C4 Relative exposure varies with the length of the rectangular light spot

D1 Device selector

D2 Control logic

D3 $X$ data buffer

D4 Y data buffer

D5 Digital to analog converters

E1

Unit $A$

E2

Unit $B$

E3 Device selector and width buffer

Digital to analog converters ( $\triangle X$ and $X$ )

E7 Digital to analog converters ( $\triangle Y$ and $Y$ ) 
Figure

E10 Integration and summing amplifiers and manual-adjust clock

E11 Programmable voltage divider 143

E12

$Z$ pulse amplifier and selectors

144

E13

Block diagram of a Tektronix 502 oscilloscope

145 
IIST OF FLOWCHARTS

Flowchart

Page

$3.1 \quad$ Line-drafting

49

4.1 Area-fill subroutine

70

4.2 Area-fill program

72

5.1 Character printing program 


\section{INTRODUCTION}

\subsection{General}

A cathode-ray tube (CRT) light-head is a lightbeam-drafting instrument using a CRT as a light source to produce drawings of precision quality on films. This instrument, controlled by a mini-computer, reduces the labour of drafting because it offers complete reliability and flexibility in symbol generation and permits full possibility for automation.

Tracing an engineering drawing or any complex graphic information requires one of man's most highly developed skills. In the past this drafting effort has depended largely upon the manual skill of experienced draftsmen.

In cartographic drafting, one of the major problems is that of the efficient lettering of maps, i.e. the addition of names, explanations and numbers with proper style, position and readability. The problem is important, because maps can contain many thousands of letters, and the work involved has had a strong influence on the editing and drawing time of the maps.

The advent of sophisticated computers has provided the necessary technology for significant advances in computer-aided design and computer-controlled graphic hardware. The result of this technological advancement 
has been to relieve engineers, architects, and cartographers from tedious, repetitive tasks through automatic drafting systems $(1)(2)(3)$. These systems produce drawings in minutes instead of the hours required for manual drafting. Furthermore, the resulting drawings can be highly accurate and of very high quality. Automatic drafting systems also allow cartographic data from a variety of sources to be combined after scaling and manipulation by a digital computer.

The main elements of a typical automatic drafting system are shown in Fig. 1.1. These consist of an input device, a controller, and a drafting table (including a drafting instrument-'pen'). Data which is mathematically based or digitized from maps can be the input. The controller reads data from the input device, interprets it and controls the movement of a drafting table, as well as the behaviour of the 'pen' on the table, in order to produce the desired drawing. The results from the drafting depend much upon the nature of the 'pen' used relative to the requirements of the drawing.

At present, there are three basic techniques of drafting that could be considered for use in automatic drafting systems ${ }^{(4)}$. The first technique uses an ink pen to draw lines directly onto paper. The second uses a scriber to remove a thin plastic emulsion coating from a transparent mylar base. The third uses a light-head, the 
focussed image of a light beam, to produce lines directly onto photographic film.

Among the above techniques, the light beam method of drafting is the most versatile and the only one to offer full possibility for automation. (See Appendix (A)). According to the method of producing a light beam, lightheads are classified into two types, namely an optoelectronic light-head and an opto-mechanical light-head. In an opto-electronic light-head, the symbolic image is produced electronically and is projected onto a film so that its quality is highly dependent upon the resolution of the light-head. On the other hand, an opto-mechanical light-head basically consists of a light source, an optical imaging system of lens, a set of mechanical masks and a light shutter. The symbolic image is produced by shaping the beam from the light source into the desired configuration through the mechanical masks and flashing it onto a film so that a high quality symbol can be produced.

1.2 Disadvantages of an opto-mechanical light-head

However, the main limitation of an opto-mechanical light-head is its lack of flexibility. Symbol masks typically contain from 6 to 96 symbols; when alphanumerics are taken into account, even 96 is often too small a number for any one cartographic overlay sheet. Thus only a limited 
number of characters or symbols can be accessed automatically and it is not easy to interchange masks in the dark with the sensitized film exposed on the table.

It will be noticed that symbol rotation is necessary if these flashed-down symbols are to be angled, and also that automatic dynamic image rotation must be fitted to maintain constant line width in all directions if a rectangular light spot is used for line drafting (see Appendix (A)). This rotation, which must be very rapid in response, requires mechanical action and this often causes eccentricity in the rotation center. For these reasons, further investigation regarding an opto-electronic light-head was most desirable. This is the main concern of this thesis.

1.3 Previous work on a CRT Iight-head for character printing In order to overcome the disadvantages of optomechanical light-heads for an automatic drafting system, a precision CRT device or opto-electronic light-head was selected as against a matrix of light-emitting diodes, because of its speed, versatility and relatively uniform illumination. (See Appendix (A)).

A precision CRT device is currently being developed at the University of Saskatchewan by the Graphic System Design and Application Group headed by Dr. A.R. Boyle. W.T. Wilkie ${ }^{(5)}$ presented his investigation of name placement 
using a CRT as a photo-typesetter. This system, provided a flexible technique which could efficiently generate characters under software control. The block diagram of this system (see Fig. 1.2) shows:

a) the precision CRT and associated electronics

b) the optical system of lens to project the image of the CRT onto the drafting film

c) the X-Y mechanism used to move the CRT unit over the film

d) the interfacing electronics used to connect the CRT unit and $\mathrm{X}-\mathrm{Y}$ mechanism to the computer

e) the computer and its control programs.

Using a 128 by 128 equivalent element matrix, symbol construction data is held in 'scan line form,' where absolute start and end coordinates of a vertical scan line are stored in the library file of the computer. The size of some entries in the library of construction data can be significantly reduced by the application of symmetry, since many characters and symbols have symmetry about one or both axes. Therefore, an almost unlimited number of symbols can be available as they are stored in digital form in the library file. Rotation of the symbol is calculated by the computer. There is no eccentricity introduced in the rotation center as often occurs with an opto-mechanical light-head. For actual character printing, the control program positions the 


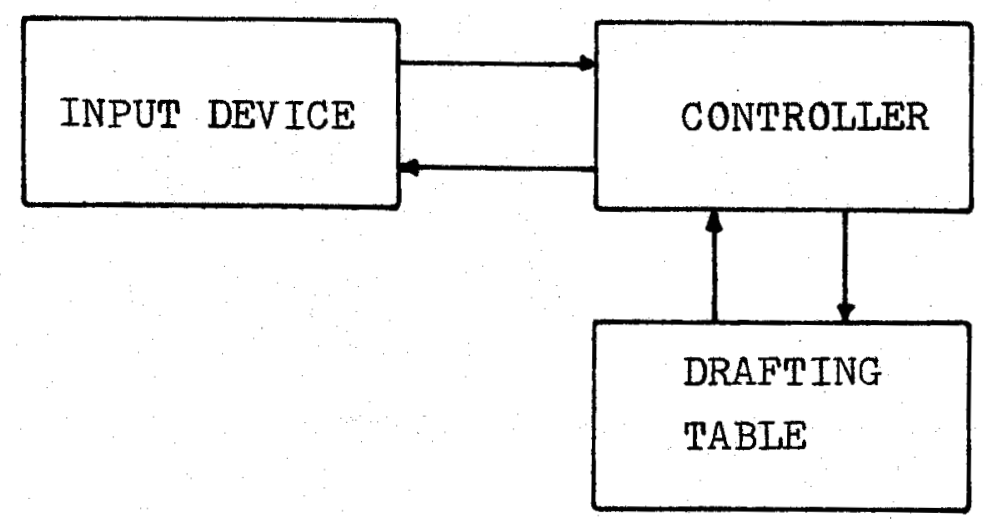

FIGURE 1.1 BLOCK DIAGRAM OF AN AUTOMATIC DRAFTING SYSTEM

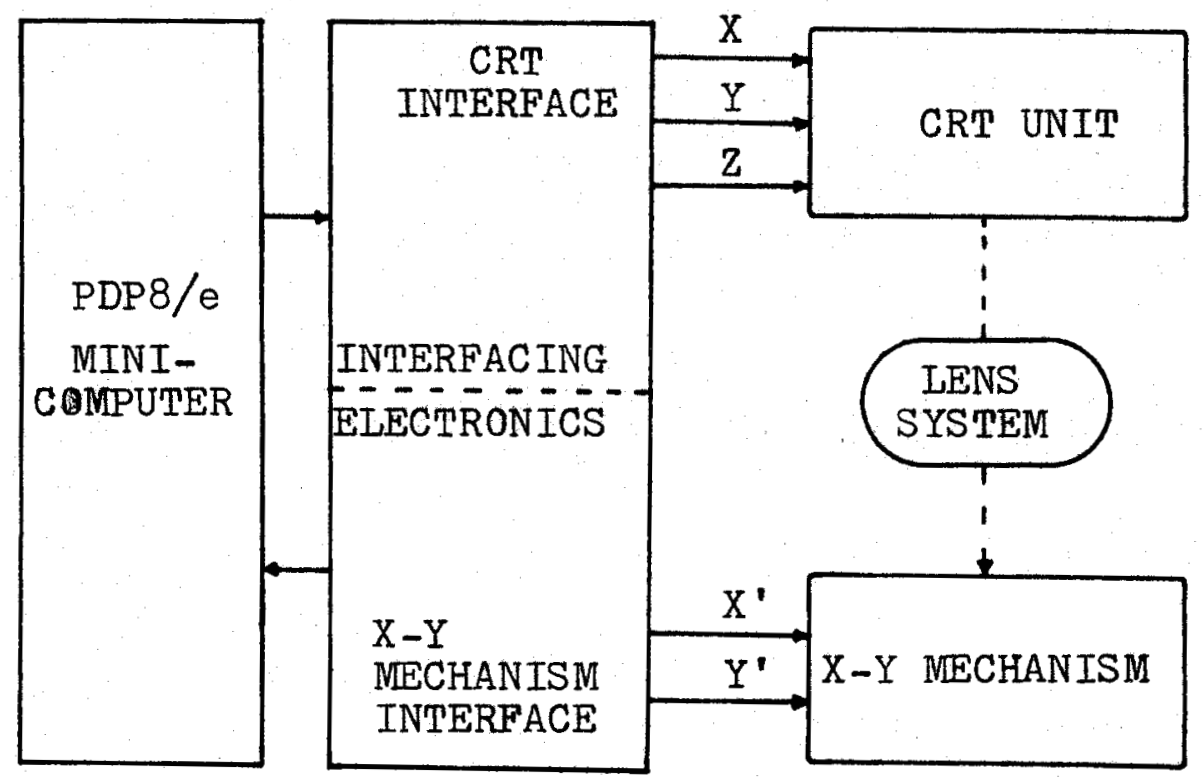

FIGURE 1.2 BLOCK DIAGRAM OF CRT PHOTO-TYPESETTER 
$X-Y$ mechanism, retrieves the character construction data from library files, decodes the construction and symmetry data and then deals with the rotation, the scaling and the printing of the character or symbol onto the film sheet. The basic concept appeared to be sound and the symbol could be generated digitally at uniform illumination without any compensation for the image intensity for different sizes, because all the points within the image were generated digitally with constant intensity. However, to modify the size, it was necessary to increase or decrease the number of unit elements within the construction data matrix. Enlargement and reduction were accomplished by interpolation to generate column and row unit elements between those specified in the construction data matrix. In addition, certain criteria had to be determined and applied in the situations of discontinuous curvature such as internal corners. It was noted that the quality of symbol generated in this way varied with the angle of rotation, because every scanning stroke of a symbol was no longer a vertical straight line as at zero degrees, but instead a series of dot elements appearing at the slope of the required rotational angle.

1.4 Objectives of the present work

The previous work on a CRT name placement system 
showed that it could be implemented to generate characters and symbols of an acceptable quality for cartography. An extension of this idea is the development of the CRT device as an opto-electronic light-head for other automatic drafting applications. The purpose of this thesis is to study the feasibility of CRT light-heads for comprehensive use in automatic drafting systems and to indicate the results from an experimental unit.

The second chapter discusses the requirements of the system and the selection of system components using a CRT as a light-head for an automatic cartographic drafting system. The third chapter introduces the line drafting techniques of the system. The interface and control required to connect this opto-electronic light-head to the PDP8/e mini-computer and Gerber 22 drafting table are then discussed.

In automatic cartography, 'area-fill' is especially applicable to the generation of colour overlays presently made by removing areas on 'peel-coat,' a difficult operation. Therefore, the fourth chapter deals with a particular aspect of using a CRT light-head for this application to save the use of 'peel-coat.' For the remaining portion of this text, character printing using the technique of 'area-fill' is evaluated and compared with Wilkie's work ${ }^{(5)}$. 


\section{SYSTEM REQUIREMENTS AND COMPONENTS SELECTION}

\subsection{General}

The general cartographic requirements for an automatic drafting system are as follows:

a) 'Well formed cartographic quality lines' must be produced It must be possible to draw squareended lines; this is particularly important when lines are relatively wide or are used to draw square features such as buildings.

b) Great flexibility of symbolization A large number of symbols including size and angle variations are to be available.

c) Precision and accuracy It must have a sufficiently good drafting resolution ( of the order of $0.005 \mathrm{~mm}$ ) to generate high quality lines and characters. Since most cartographic drawings are printed in colour using conventional lithographic techniques, preparation of a chart for printing involves making a separate negative for each colour required. A printing plate is then constructed for each colour. For proper colour registration, the relative accuracy of each colour overlay must be maintained. Using a light-head as a 'pen' for a drafting 
system, cartographic information can be drawn either by each line individually or by an image flashing technique. In the CRT Iight-head system, the former technique is applied for 'line drafting' while the latter technique is used for 'area-fill' and 'character printing.' However, when first considering the requirements and the selection of components of the system, the line drafting technique is emphasized because it includes the major requirements and is the dynamic state of the image flashing technique.

2.2. Requirements of the system

The overall line drafting requirements are complex and some are yet to be exactly determined. For the initial stage of the development and thus for the purpose of this thesis, it is proposed to have a rectangular light image, generated on the screen of a CRT, as a scan-line for cartographic line generation. (Fig. 2.1). A rectangular light spot is selected from other configurations because of its advantages of simplicity, compatibility in producing square ends, and relatively uniform exposure. In addition, it is easily generated on a faceplate of a CRT. This image (scanline image) is then focussed onto a lithographic photosensitive sheet through an optical lens. The CRT which is mounted on the drafting table would move continuously as for 'pen type' drafting. 


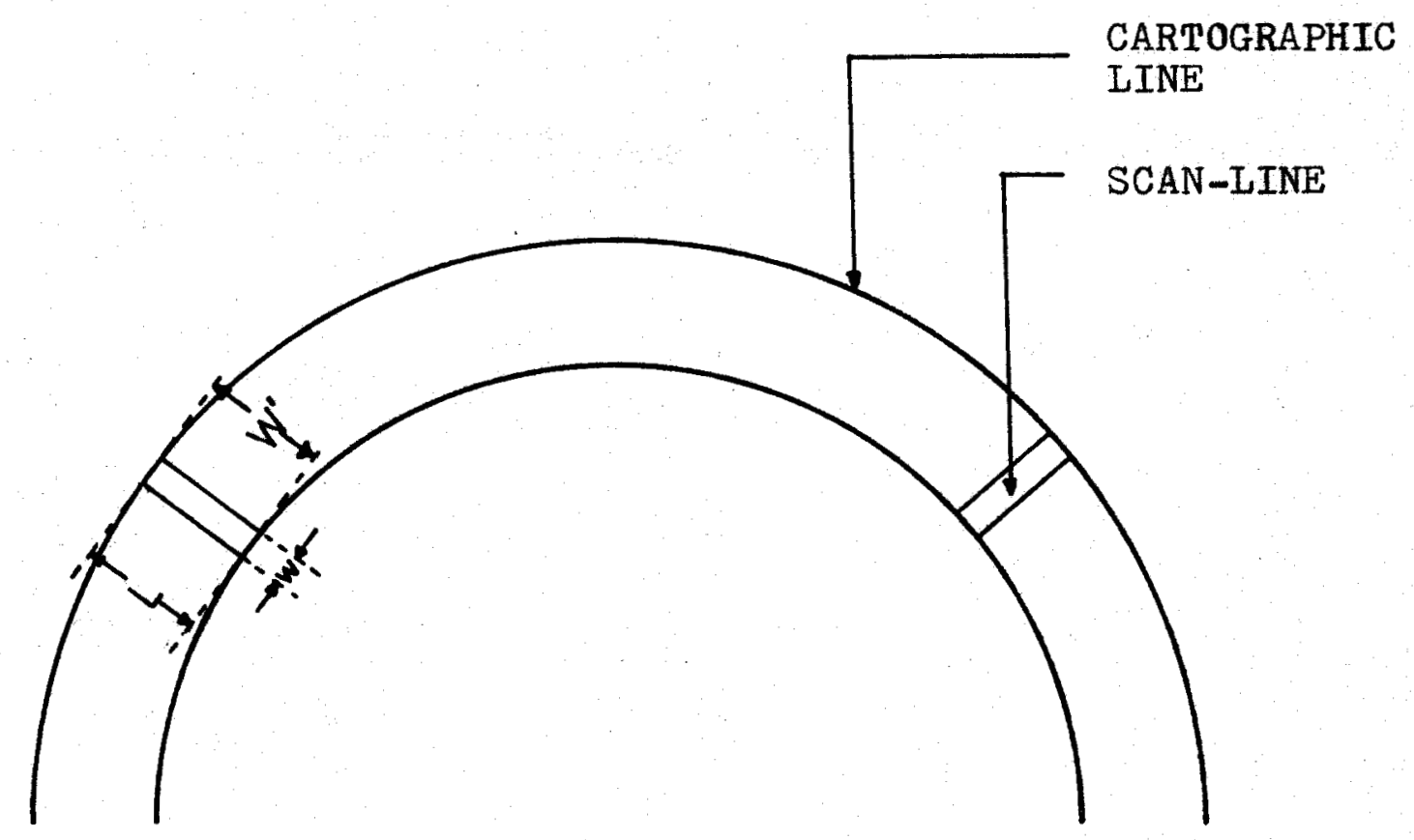

$L=$ THE LENGTH OF A SCAN-IINE

$W^{\prime}=$ THE CARTOGRAPHTC LINE WIDTH $=$ L

$w=$ THE WIDTH OF A SCAN-IINE

FIGURE 2.1 CARTOGRAPHIC LINE 
The width of the scan-line ' $w$ ' could be varied by software. This is done by generating the scan-line point by point while the CRT light-head remains stationary at a position above the cartographic line position for the whole period of a scan-line generation. The drafting speed of the system would then be extremely low. On the other hand, if a single scan-Iine were repeatedly generated by hardware, an approximation to a rectangular light spot with a constant width that of the CRT beam width, would be obtained and the speed of the system would be increased. Ideally the width ' $w$ ' of the rectangular light spot should be kept small so that the effect of multi-exposures at the intersection point does not need to be considered when lines meet. As this dimension is decreased, however, the intensity of the projected image must be increased to maintain the drafting speed. Further details about this effect can be found in Appendix (C). In other words, only a high intensity CRT can be applied for use as a light-head in this manner.

For the purpose of maintaining constant cartographic line width when moving in any direction, automatic dynamic image rotation is another important requirement for the system when using a rectangular light spot for line tracing. This rotation must be very rapid in response, in order not to affect the speed of line drafting, (i.e. the $X-Y$ motion should not have to await the angular one). 
Moreover, the center of the rectangle must remain stationary as it is being rotated, and its length must be kept constant at all angles of rotation. The net result of any errors in rotation is that junctions of lines are not correct. Data which is mathematically based or digitized from maps can be used as an input to the system. In cartography, a number of line widths are required, ranging from $0.1 \mathrm{~mm}$ to $0.5 \mathrm{~mm}$. The width of a drafted line must be selectable and accurate to $0.005 \mathrm{~mm}$. Furthermore, since cartographers normally seem to be able to accept lines which are slightly inaccurate as long as they are smooth, smoothing routines should be available that give well shaped cartographic lines. As an approximation, the minimum curvatures along a topographic contour and a coastline might be $0.5 \mathrm{~mm}$ and $0.1 \mathrm{~mm}$ respectively ${ }^{(6)}$. Hence, for line regeneration, an unexpectedly small radii of curvature or unwanted data in the form of spikes to the side or along a line due to the errors of digitizing operation have to be removed. This may be done by simply linear interpolation or by calculated circular or cubic spline-fit processes. Each method has its own advantages and disadvantages in different applications.

The requirement of intensity control is necessary when high quality lines are desired. Since the speed of the drafting table varies considerably from its initial 
starting speed to its maximum velocity along a line, the exposure time for line drafting also varies from point to point. Therefore, some method of control is needed to vary the intensity of the projected image so as to have a uniform exposure for a whole line. Fortunately, such a control need not be precise because of the large latitude of $\mathrm{film}^{(4)}$. However, it must have a large operating range and have a high enough speed of response to follow the drafting unit as its speed varies.

High speed of line drafting is also another requirement of the system. It is dependent upon the intensity of a CRT, the aperture of the optical lens, the sensitivity of a film and the velocity capability of the drafting table. To optimize these system parameters separately for drafting speed would adversely affect the quality, so care must be taken to reach a good compromise. Each of these components will be discussed in the next section of this chapter. As mentioned in Appendix (A), the drafting table of the system must be located in a controlled atmosphere darkroom, of suitable dimensions, to maintain film and drafting table at constant linear dimensions.

In short, the automatic controls that are required for the system are summarized below:
a) Rotation of a scan-line image Control the rotation of the scan-line image to follow the 
direction in which the drafting table will proceed.

b) Cartographic line width Control the length dimension of the scan-line image. The change in width of a cartographic line should not affect the drafting speed.

c) Iine smoothing routine Regenerate a new smoother digital line from the line in the digital cartographic data-base.

d) Intensity of projected image Vary the intensity of the projected image according to the speed of the drafting table.

2.3 Selection of system components

In this section, the major characteristics of the system components are explained and specified. The components were chosen according to system requirements, cost and availability.

2.3.1 Optical lens

An optical lens is used to reduce the image from the screen of the CRT light-head onto the photo-sensitive material. For a given exposure of film, the maximum drafting speed is proportional to the aperture of the optical lens being used. Lenses for photographic work are usually 
marked with the $\mathrm{F} /$ number, which is the relative aperture of the lens. The relative aperture indicates the amount of light energy that a lens can transmit; this is, two lenses with the same $F /$ number will transmit the same amount of light and therefore give equivalent exposures regardless of focal length ${ }^{(7)}$. If two lenses have a different $F /$ number, they will give different exposures. For example, the aperture area of an F/1.4 lens is four times that of an F/2.8 lens of the same magnification, and therefore gathers four times more light. The formula for the relative aperture is

$$
\mathrm{F} / \text { number }=\frac{\text { focal length }}{\text { diameter }}
$$

Generally, increasing the relative aperture of lens also increases lens aberration.

Another important parameter is the depth of focus of a lens. It is the distance through which the focal plane can be moved without a detectable change in image quality. The larger the lens aperture (lower $\mathrm{F} /$ number), the shorter is the depth of focus.

The depth of field of a lens is defined as the region within which all object points will image sharply. It is dependent only on the diameter of the aperture and not its focal length.

The lens magnification is a number which signifies 
the image-to-object ratio of a lens system. The smaller this magnification is, the greater the intensity of the image on the film. Therefore, higher writing speeds can be achieved. Since there are significant variations in the flatness of the large active drafting area relative to the carriage assembly, an F/2.5 lens with a focal length of $6.5 \mathrm{~mm}$ was selected for the system in order to obtain good depth of focus. (If depth of focus could be sacrificed, an $F / 1.4$ lens with shorter focal length would be preferable for higher drafting speeds.) Another reason for using a higher $\mathrm{F} /$ number lens is that it can provide higher projection density. This property will be discussed in the following section. It should also be noted that the lens system should be mounted in such a way that the position of the lens plane is always parallel to the drafting material.

\subsubsection{Photographic film}

Photographic films vary in size, speed, contrast, spectral response, resolution, and cost. Therefore, some of these properties should be clarified.

The D- $\log E$ curve is frequently used to describe the characteristics of a photographic film. The relation of density to exposure is presented in the curve in which the optical density is plotted against the logarithm of the exposure. The density (D) is a measure of the transmissive 
or reflective properties of the exposed and developed image. It is defined as the negative of the base 10 logarithm of the transmission (or reflection) ratio. For example, a perfectly reflecting or transmitting surface has a density of 0.0. A 0.1 percent reflecting or transmitting surface has a density of 3.0 units. With a sufficiently extended range of exposure, the $D-\log E$ curve consists of four parts in which the slope, or gradient, first increases, then becomes constant, then decreases, and finally becomes negative and heads downward ${ }^{(8)}$. (Fig. 2.2). The slope of the straight-line portion of the curve is often referred to as latitude. Usually, high contrast film having a very steep slope or wide latitude is best for line drafting (4). Using this kind of film the requirement for the intensity control of the system would be easily achieved, as the film is exposed well into the third region of its D-log E curve, so that a change in exposure will not produce significant changes in film density.

$\mathrm{H}$ and $\mathrm{D}$ (Hurter and Driffield) curves are similar to the $D-\log E$ curves and determine the maximum and minumum density values for any given material. Figure 2.3, adapted from Reference (9), presents a set of $\mathrm{H}$ and $\mathrm{D}$ curves for Kalvar type 10 material, exposed and processed under typical conditions. These curves represent the density at various projection system apertures. It will be noticed that the 


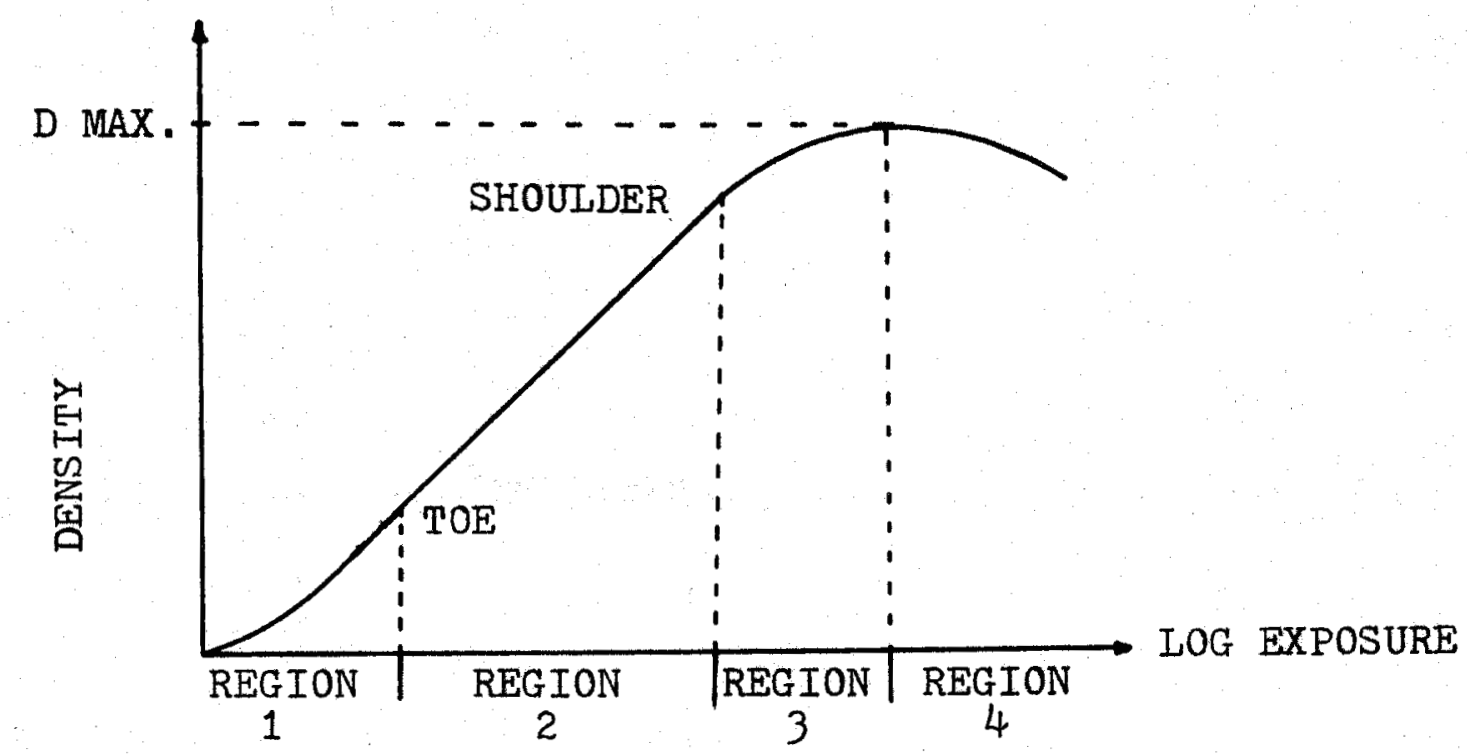

REGION 1: REGION OF INCREASING GRADIENT

REGION 2: REGION OF CONSTANT GRADIENT

REGION 3: REGION OF DECREASING GRADIENT

REGION 4: REGION OF REVERSAL

FIGURE 2.2 THE D LOG E CURVE

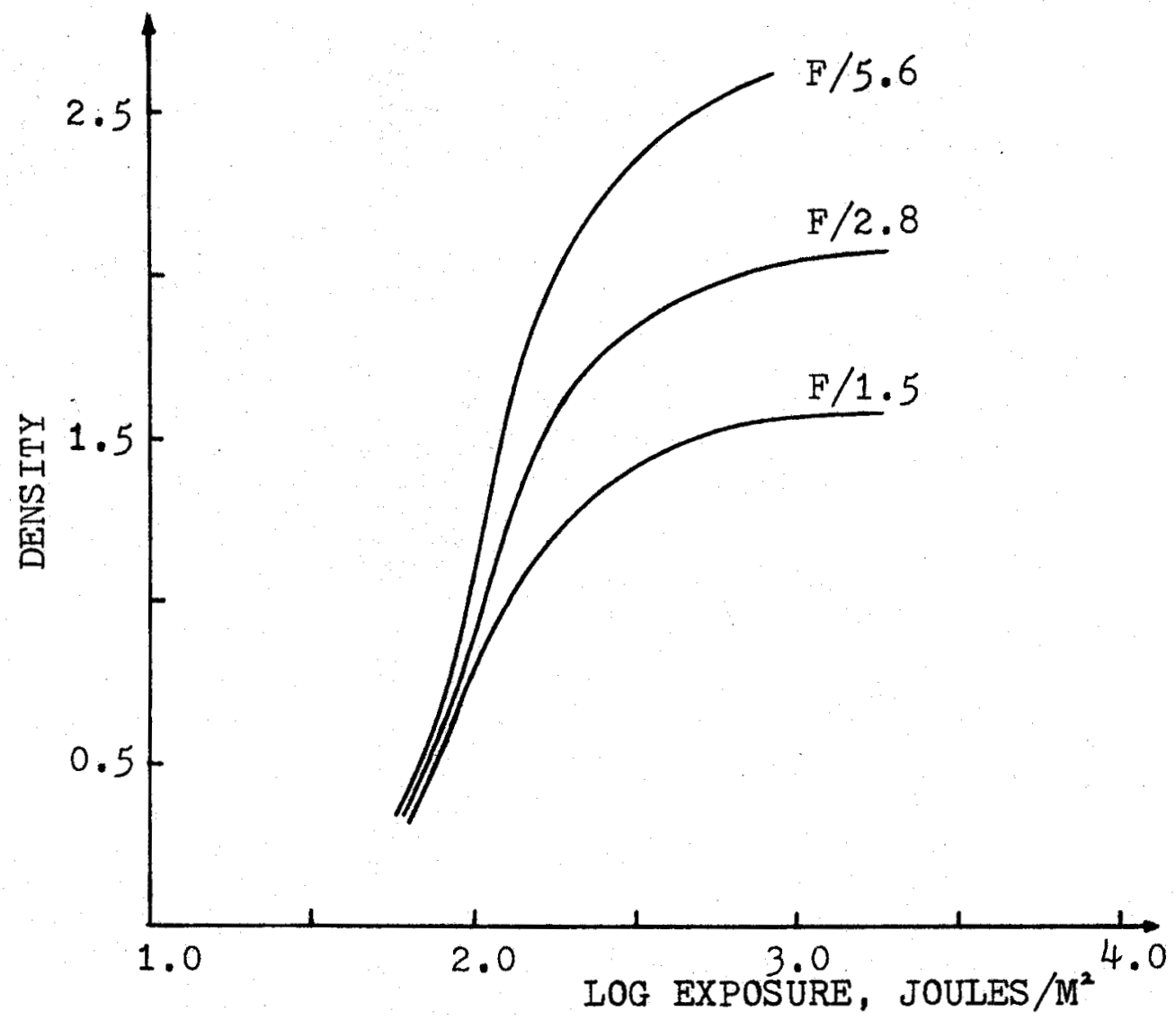

FIGURE 2.3 H \& D CURVES FOR TYPE 10 KALVAR 
projected density increases with the $F /$ number. The reason for this phenomenon is due to the failure of the reciprocity law ${ }^{(8)}$. The projected density depends upon the actual values of intensity and time and not on the product of the two. Reciprocity failure varies with the characteristic of the emulsion of a photographic film. As mentioned before, this variation of the density with the $F /$ number is considered in the choice of an optical lens for the system.

The ASA film-speed rating signifies the exposure requirements for general pictorial photography where the light has a very wide colour spectrum. Since CRT phosphors emit light over a narrow spectrum, the ASA speed cannot be used to accurately predict the exposure requirements for a CRT light-head system. Slower speed film is desirable because it has smaller grain size and therefore better resolution. The GAF ECONOLINE P4 film was chosen for the prototype system because of its characteristics of high contrast and appropriate spectral response. of course, other parameters such as cost, speed, resolution, dimensional stability, ease of handling and processing, and the degree of intermittency of the film were also taken into account. The degree of intermittency will be discussed in Section 3.6.2.1.

\subsubsection{Drafting table}

The quality of map which can be obtained depends appreciably on the quality of the mechanism of the drafting 
table. There is always much argument in cartographic circles about the relative advantages of the incremental method and the analog feedback method of controlling table position. Analog servo-mechanisms can usually be run at higher speeds than incremental ones and will maintain smoothness although small oscillations may be apparent at certain angles of lines. However, the analog mechanisms were not chosen because they need more frequent adjustment as they are not 'definite' in control and also because they are not as accurate as the best incremental mechanisms, (they are subject to drift, and positioning accuracy depends on stability of control of voltage sources).

Since only a few vector directions are available, curved lines generated by an incremental plotter tend to have a stepped appearance. The jaggedness can be reduced to negligible proportions by using a mechanism with very short step lengths, but this tends to slow down operation because the plotting head must generally come to rest at the end of each step. This problem can be reduced by having a velocity-control feature so that the drafting head, instead of coming to rest after each step, is simply accelerated and decelerated sufficiently to negotiate changes in direction in the line being plotted.

Another important feature of the drafting table on which a CRT light-head is to be mounted is its weightcarrying capability. In other words, the large mass of the 
CRT must be mountable on the table without causing any deterioration in performance.

After reviewing the requirements as mentioned above, the Gerber 22 incremental drafting table with a step size of $0.0127 \mathrm{~mm}(0.0005$ inch $)$, and speeds of up to $15 \mathrm{~cm}$ per second, was selected. Furthermore, this table had a precision flatness over the plotting area of $1 \mathrm{~mm}$, and a vacuum hold-down system to ensure the flatness of a drafting material. The Gerber 32 would have been even better but it is more costly.

2.3.4 CRT unit and its associated electronics

In the application of line drafting with a CRT light-head, the cartographic requirements restrict the selection of an appropriate CRT unit and its associated electronics. Basically there are two types of CRT units: 1) electrostatic, and 2) electromagnetic, determined by the deflection method employed. One of the main disadvantages of all electromagnetic deflection systems is their slow scanning speeds as compared to the electrostatic tubes, due to the recovery time limitation of the yoke. However, they produce a smaller spot size, they have higher deflection sensitivity and they can produce a high intensity beam with a relatively low voltage supply. They are therefore more appropriate for the system.

The beam spot size, and the type of phosphor 
employed in the CRT, are of major importance in the lighthead unit. The beam spot size determines the resolution of the tube. In order to obtain the necessary symbol and line quality, a resolution of at least 40 lines per $\mathrm{mm}$ is required to be obtained on the drafting material. The brightness of a CRT is a function of the energy in the beam and the efficiency of the phosphor. In other words, important factors in selecting a phosphor for a CRT unit are screen efficiency, persistence and spectral energy distribution. The screen efficiency is determined by how much of the beam energy is converted into light energy. The brightness is proportional to the beam energy up to the point where saturation occurs. Saturation may be caused by voltage, current or heat ${ }^{(10)}$. The persistence is defined as the time required for the intensity on the screen to drop to $10 \%$ of its initial value after the removal of the beam of electrons. Long persistence can be used to increase the exposure time when a lower scanning rate is employed. However, the disadvantages of the long persistence screens are that they suffer from low light output and have low resistance to burn. The P1 phosphor, which matches the emulsion sensitivity characteristic of the selected film, was chosen due to its relative high resistance to burn, high efficiency and high apparent resolution.

The physical weight and the screen size of a CRT 
unit are also to be considered in selecting a light-head. In addition, the associated electronics should be selected to provide acceptable linearity, repeatability and stability $(5)$. There are some CRT subsystems such as the CELCO DS5-50-0 ${ }^{(11)}$ and the BETA PD900 (12) that meet the above requirements. They are available but at a cost of around $\$ 15,000$. The TEKTRONIX model 502 electrostatic deflection oscilloscope with the type T5021P1 tube was actually used as a test device for the experimental proto-type system. Although insufficent in quality and light output for a final unit, the TEKTRONIX 502 (P1 phosphor and approximate $0.6 \mathrm{~mm}$ spot size) (13) was practical for the basic prototype test and software development. 


\section{IINE DRAFTING}

\subsection{General}

In this system, a Digital Equipment Corporation PDP8/e mini-computer with a magnetic disc storage unit and 9-track magnetic tape unit, was used as a controller. The advantage of using a mini-computer for a controller was that the hardware required for the control could be simplified by having the computer program handle several of its functions. Such functions as data transfer, data storage and calculation can also be handled. These factors, together with the complexity of the required hardware, must be considered when determining the optimum hardware/software combination for a controller.

In line drafting, it is necessary to generate a scan-line image on the screen of the CRT for the whole time that the carriage, on which the CRT is mounted, is moving. There are two basic approaches to vector generation: either points on a straight line are computed digitally and the line is drawn as a series of dots, or an analog circuit is employed to move the CRT beam continuously from the starting point to the ending points of the scan-line. Digital linescanning algorithms can be implemented in software, using point-plotting techniques to intensify each dot on the scanline. Analog generators involve more sophisticated 
electronic circuits and this results in an increase in cost. It was found, however, that there was insufficient time to pass data between the drafting table and the mini-computer to maintain continuous drafting when the scan-line image on the CRT light-head was being generated by software. Therefore, a hardware scan-line generator had to be used for line drafting. Furthermore, it provided a discernably smoother scan line.

The control of scan-line rotation when drawing curved lines was achieved simply by the compilation of a 'look-up table.' The rotational angle was calculated by the mini-computer, so that positional accuracy could be obtained without cumulative error, as usually occurs with a hardware feedback control. Further details will be given in Section 3.3.1.

In the intensity control, the equivalent velocity of the drafting table can be calculated by either software or hardware. The software approach was rejected because it involved too much computer time.

\subsection{System block diagram}

A block diagram of the complete experimental drafting system is shown in Fig. 3.1. It consists of the control unit (a PDP8/e digital computer, a magnetic disc storage unit and a 9-track magnetic tape unit), the drafting 


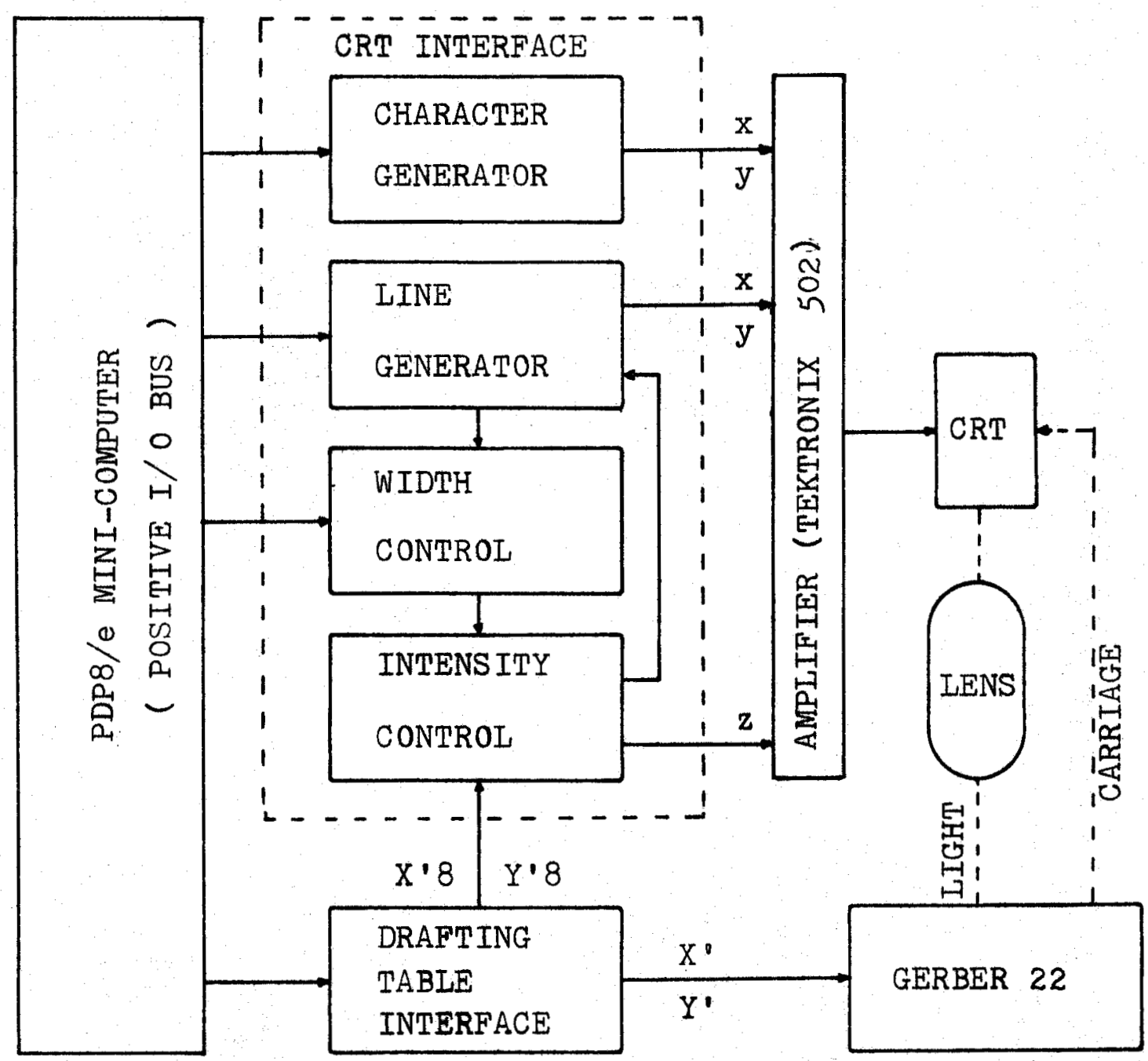

FIGURE 3.1

BLOCK DIAGRAM OF INTERFACES AND CONTROL FOR THE CRT IIGHT-HEAD 
table unit (a Gerber 22 drafting table and its PDP8/e interface), the CRT light-head unit (Tektronix 502 oscilloscope), and the optical lens unit. (See Figures 3.2 and $3.3 a, b, c, d$ ).

The drafting table interface, manufactured by Dynamap (Fig. 3.3c), is designed for the Gerber 22 drafting table which is controlled by a PDP8/e mini-computer to give a maximum speed of the table of about $15 \mathrm{~cm}$ per second with acceleration and deceleration control. It receives the incremental line data from the PDP8/e controller and converts them into pulse trains for both the $X^{\prime}$ and $Y^{\prime}$ motor axes of the drafting unit. The frequencies of the $X^{\prime}$ and $Y^{\prime}$ pulse trains govern the velocities of the drafting table in the $X^{\prime}$ and $Y^{\prime}$ directions respectively. In addition, another pair of signals, $X \cdot 8$ and $Y^{\prime} 8$ are available to be employed for the light intensity control. The frequencies of $X^{\prime} 8$ and $Y \cdot 8$ are equal to eight times the frequencies of $X^{\prime}$ and $Y^{\prime}$ respectively. The CRT interface can be divided into four units, namely the character generator, the line generator, the width control and the intensity control unit. However, the character generator unit, which was designed by W.T. Wilkie (see Appendix (D)), is treated only as an auxiliary unit in line drafting. This unit is included in this block diagram only because of its applications in the 'area-fill' and 'character printing' techniques. The CRT interface generates analog voltages representing the desired $X-Y$ coordinates on 
the screen of the CRT and the $Z$ pulse (unblanking pulse) for dynamic scan-line generation.

The cathode-ray tube and the optical lens are mounted on the carriage of the drafting table as shown in Fig. 3.2. Photographs of the basic components of the complete drafting system are shown in Figures $3.3 \mathrm{a}$ to $3.3 \mathrm{~d}$.

\subsection{Line generator and its control}

An expression of a straight line can be given in the following formulae in an integral form:

$$
\begin{aligned}
& X=X_{i}+\int \frac{\Delta x(t)}{T} d t \\
& Y=Y_{i}+\int \frac{\Delta y(t)}{T} d t
\end{aligned}
$$

Where $X_{i}$ and $Y_{i}$ are the starting locations of the $X$ and $Y$ axes respectively, $\Delta x(t)$ and $\Delta y(t)$ are the vector components of the straight line in the $X$ and $Y$ directions respectively, and $T$ is the time period during which the line will be traced. Figure 3.4 shows the block diagram of the line generator in the CRT interface unit. On receiving the data $\left(X_{i}, Y_{i}, \Delta x\right.$ and $\left.\Delta y\right)$ of the straight line from the PDP8/e minicomputer, the $X$ and $Y$ ramps are generated and sent to the corresponding $X$ and $Y$ deflection amplifiers (Tektronix 502 oscilloscope). The amplitude and polarity of the $X$ and $Y$ ramps determine the length and direction of the line, and 


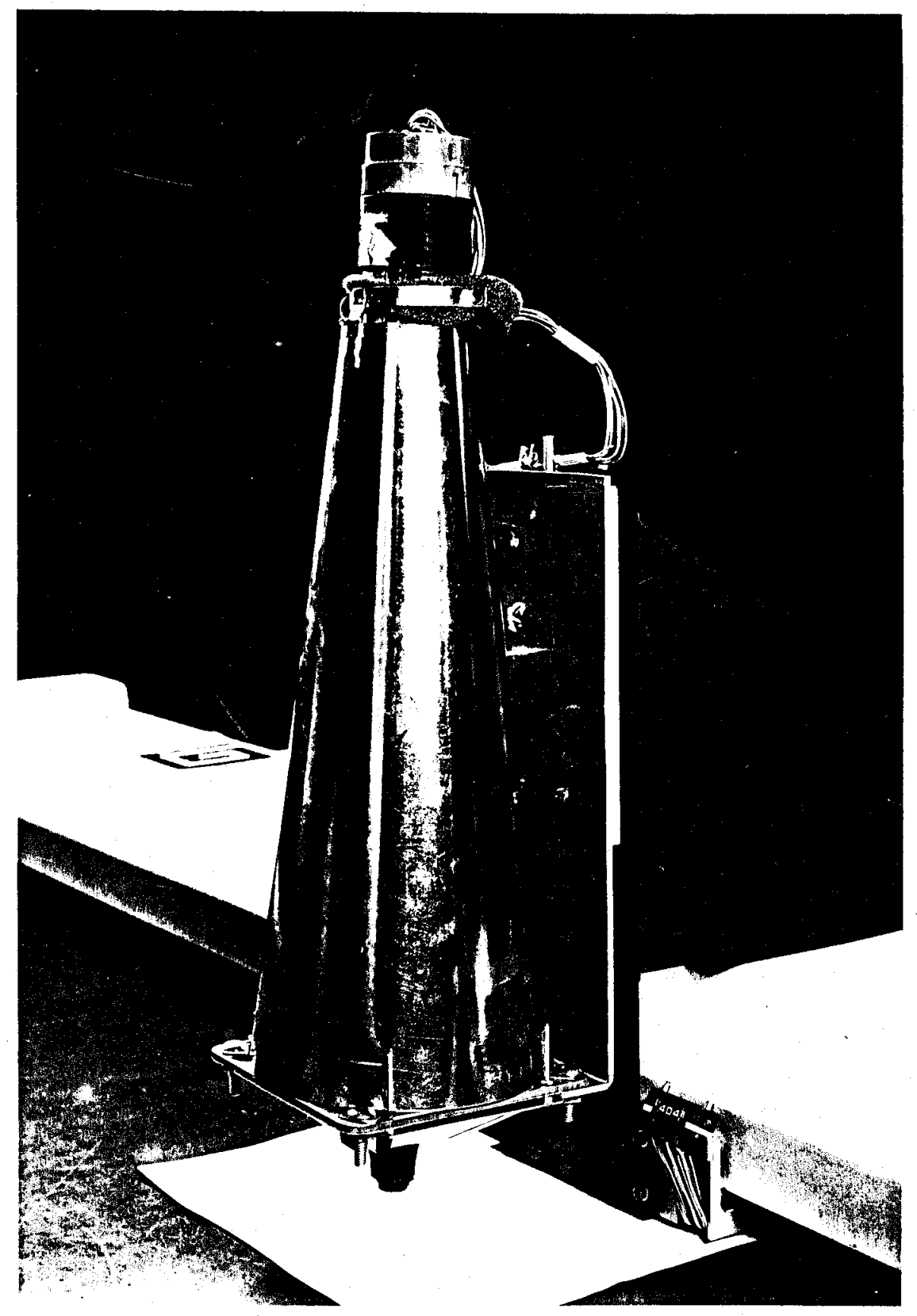

FIGURE 3.2 CRT LIGHT-HEAD 


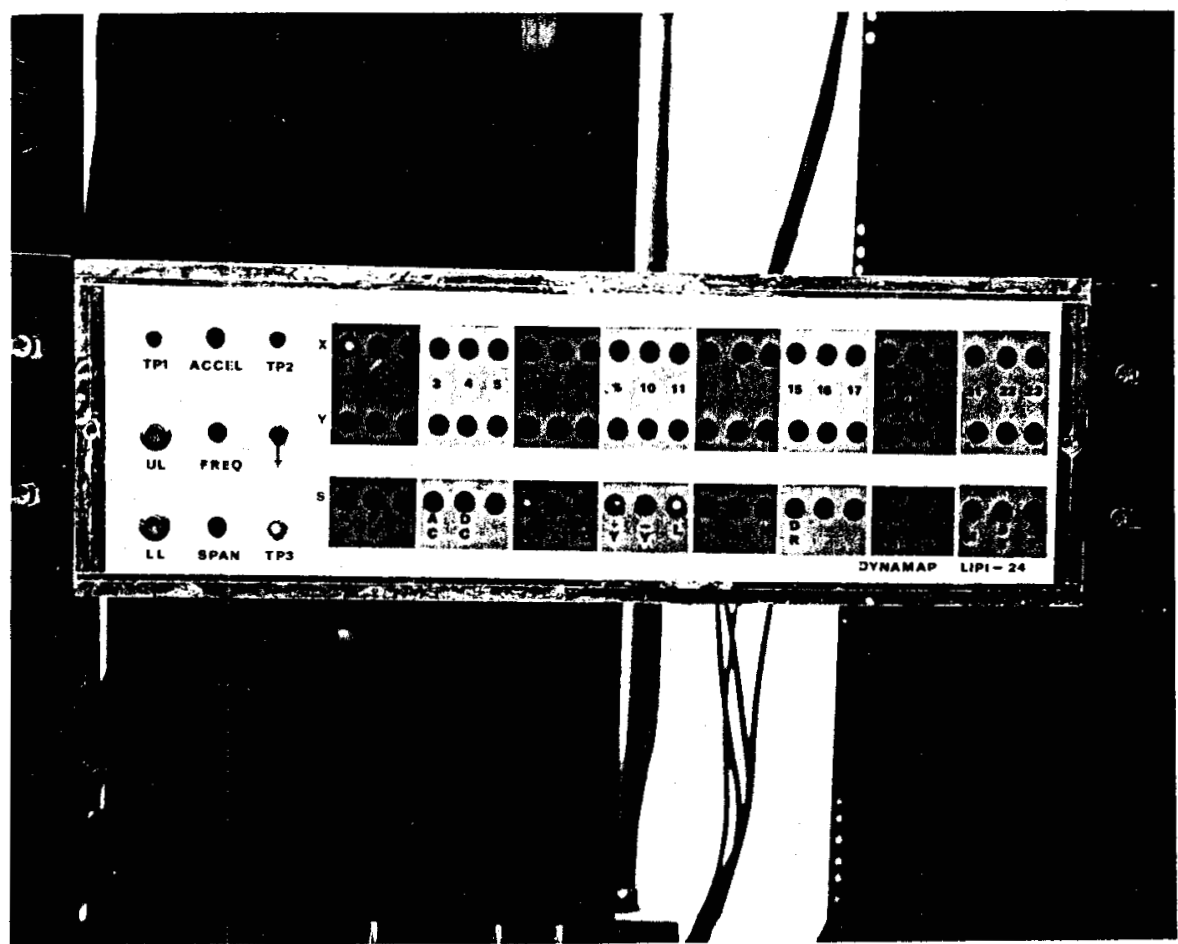

FIGURE $3.3 \mathrm{C}$ INTERFACE UNIT OF GERBER 22 TABLE

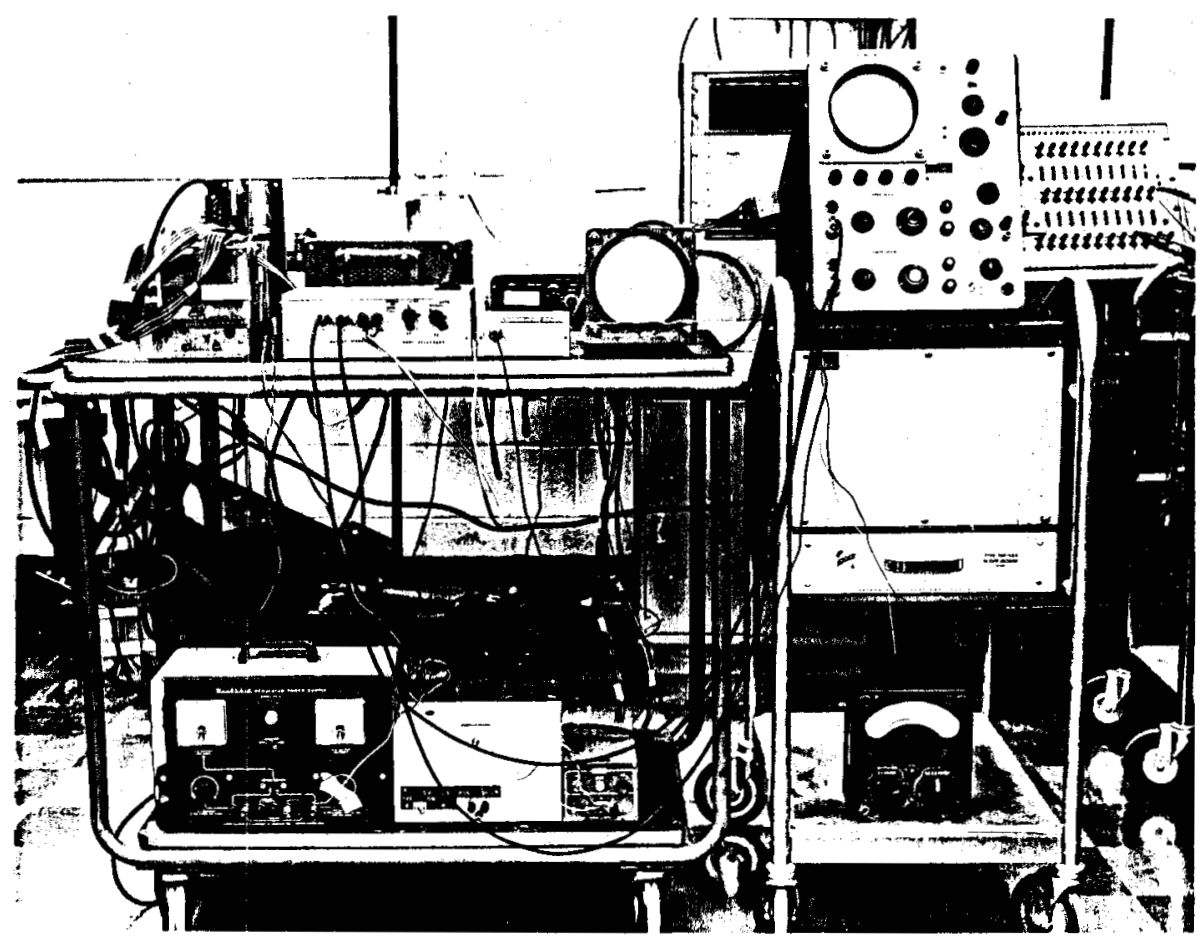




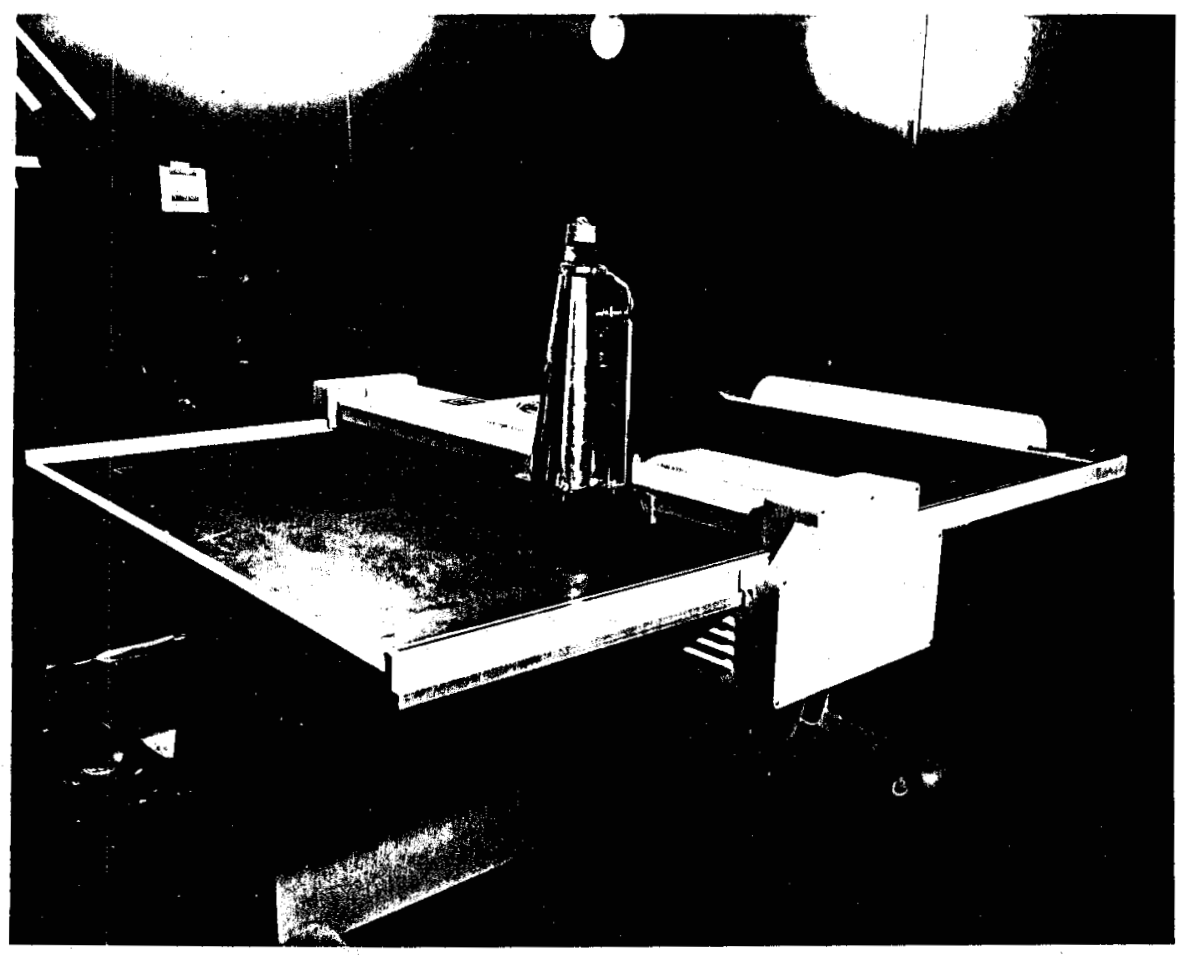

\section{FIGURE $3.3 \mathrm{a}$ CRT LIGHT-HEAD MOUNTED ON GERBER 22 TABLE}

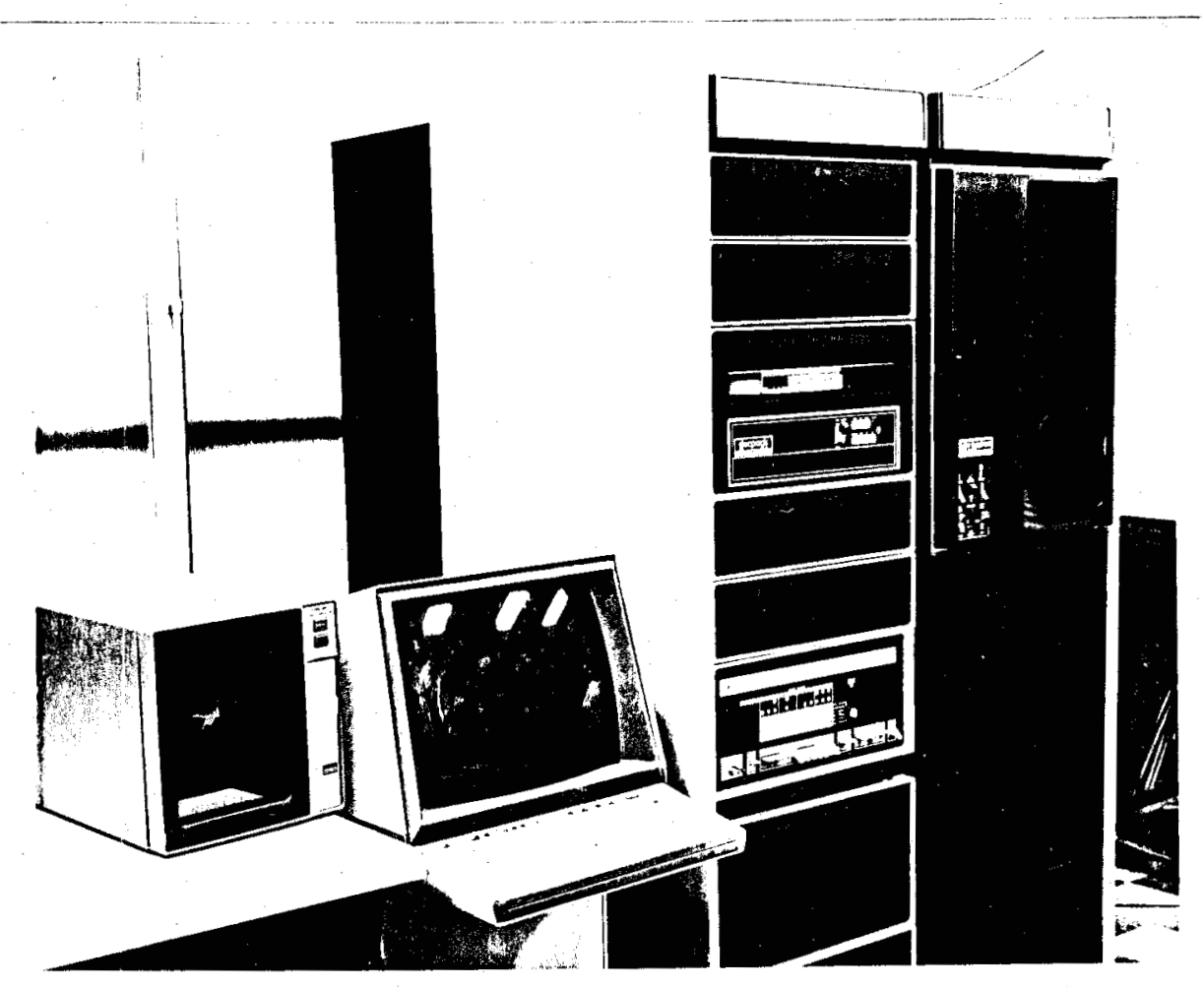

FIGURE $3.3 \mathrm{~b}$ D.E.C. PDP $8 /$ e MINI-COMPUTER 
the d.c. levels of the $X$ and $Y$ ramps determine the starting coordinates of the line on the screen. While the line is being generated the $Z$ pulse is unblanked, producing the visible line.

For each $X$ and $Y$ ramp generator, two digital to analog converters and two registers are used in addition to the input decode logic. The binary data corresponding to the initial position $\left(X_{i}, Y_{i}\right)$ are received from the computer and stored in the $\mathrm{XB}$ and $\mathrm{YB}$ registers respectively. The binary data specifying both the direction and length of the $\Delta \mathrm{x}$ and $\Delta \mathrm{y}$ components are stored in the $\mathrm{XA}$ and $\mathrm{YA}$ registers respectively. These registers drive associated digital to analog converters. The integrating timing unit, triggered by the signal from the intensity control unit, provides an integrating timing-gate signal of constant duration for the on/off control switches of the integrators.

Figure 3.5 shows the circuit of an integrator. After the digital to analog converter voltage has been applied to the integrating amplifier and the switch is opened, the amplifier output will start moving linearly. The rate at which the output moves will be determined by the digital to analog converter output voltage, amplifier gain, and the time constant $R C$. The ramp signal is then added to the initial position voltage in the summing amplifier. The signal is then routed to the deflection amplifier for a 


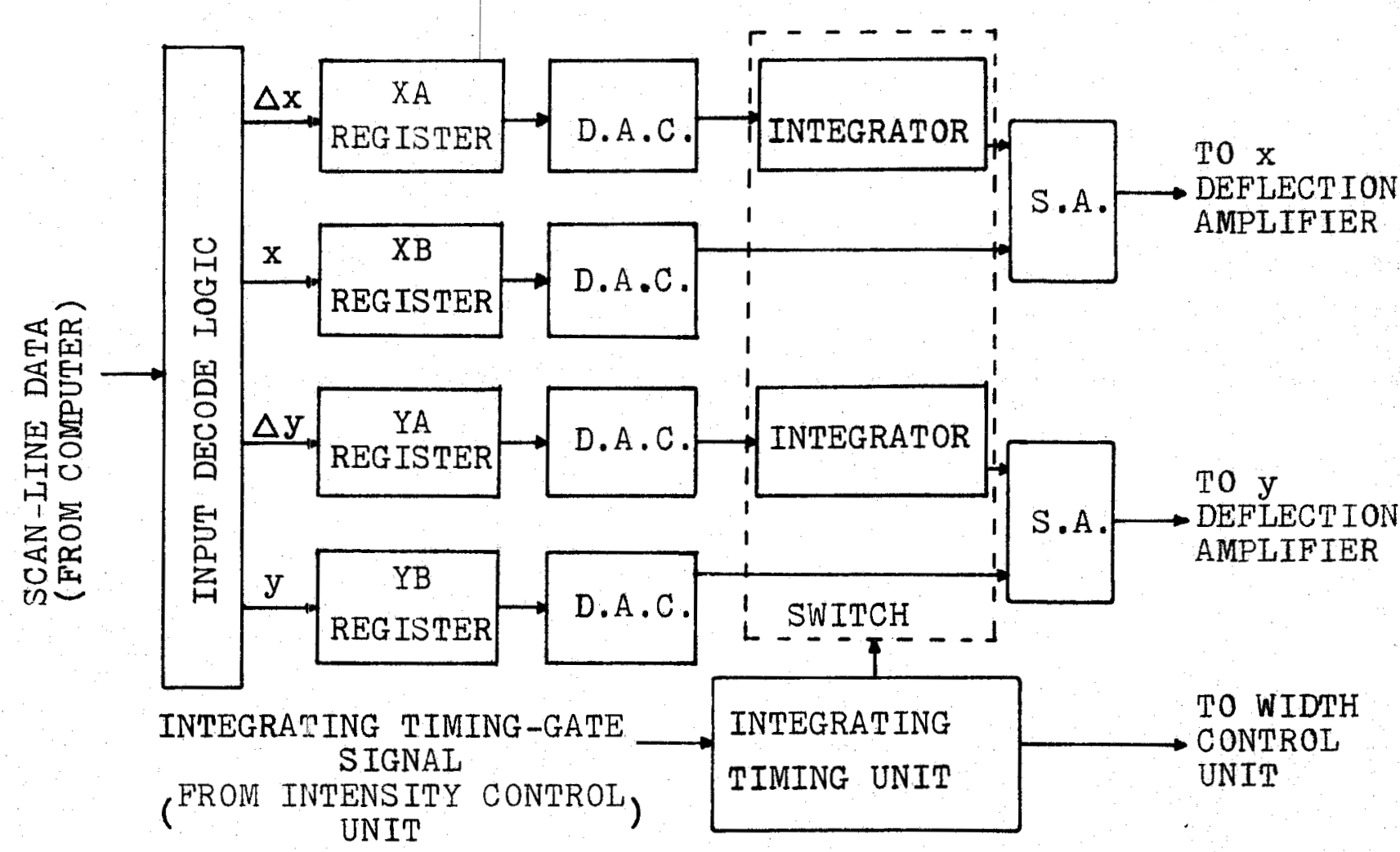

D.A.C.= DIGITAL TO ANALOG CONVERTER; S.A.= SUMMING AMPLIFIER

FIGURE 3.4 BLOCK DIAGRAM OF THE LINE GENERATOR UNIT

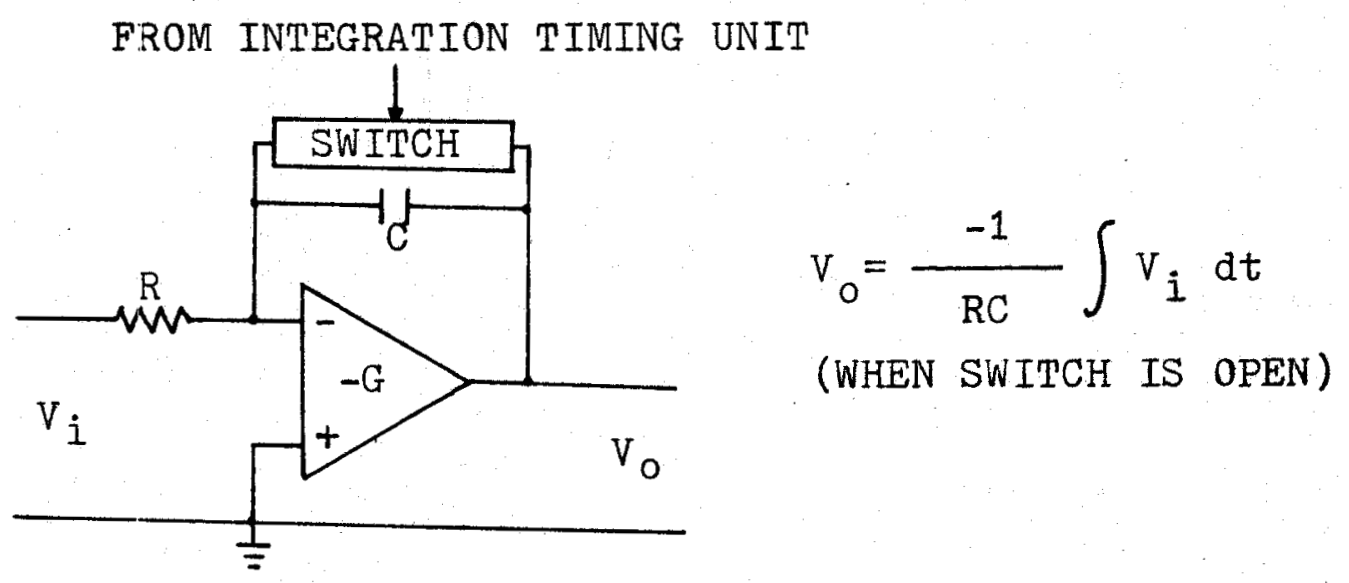

FIGURE 3.5 INTEGRATOR 
Iine generation.

The high speed of the integrators requires fast response from the deflection amplifiers and switching circuits. A disturbance, or ringing, is produced at the start of ramp generation by the transient response of the integrating amplifier. The line would then appear quite crooked at both ends unless the $X$ and $Y$ integrators, amplifiers, and switches have identical lags. A 'running start' technique, generating a ramp signal somewhat longer than the line requested, was used to avoid this problem. This method is used in the selection of the scan-line length as explained in Section 3.3.2.

\subsubsection{Rotation control}

When drawing a line on the drafting table, the direction of the line depends on the angle that the line makes with the horizontal ( $\left.X^{\prime}\right)$ axis. This angle $\theta$ is equal to $\tan ^{-1} \Delta Y^{\prime} / \Delta X^{\prime}$. (See Fig. 3.6a). In order to retain the same line width in all directions of drawing, the angle $\delta$ formed by the scan-line image with the $Y$ axis of the screen of the CRT light-head has to be equal to the angle $\theta$. This rotation control is achieved by software programming.

As mentioned above, the four values, $X_{i}, Y_{i}, \Delta x$ and $\Delta y$, are required for the scan-line generation. By varying this data according to the angle $\theta$, an 'all direction. scan-line can be obtained directly. For a certain length of 
scan-line, a look-up table holding these four values in three degree increments is, therefore, compiled in the scanline generation table. This table can be reduced in size by taking advantage of symmetry in the scan-line generator. (See Fig. 3.6b).

On receiving incremental data, $\Delta X^{\prime}$, and $\Delta Y^{\prime}$, the computer first calculates the angles $\theta$ in which the drafting will proceed, looks up the values of $\mathrm{X}_{i}, \mathrm{Y}_{i}, \Delta \mathrm{x}$ and $\Delta \mathrm{y}_{i}$ and sends them to the line generator for scan-line generation, and then transfers the $\Delta X^{\prime}$ and $\Delta Y^{\prime}$ values to the drafting table interface for the line drafting. In this way, whenever drafting starts, the scan-line image on the light-head has already been adjusted for the direction of the line.

3.3.2 Width control

Figure 3.7 shows the block diagram of the width control unit in the CRT interface. Whenever the $X$ and $Y$ deflection amplifiers receive the input ramp pulses, an unblanking pulse is generated from the width control unit. The width of this unblanking pulse determines the duration of the visual image on the CRT and thus the length of a scan-line.

As shown in the diagram (Fig. 3.7), a reference ramp signal is generated from the integrator according to the integrating pulse coming from the line generator unit. The maximum amplitude of the ramp signal is set to positive 


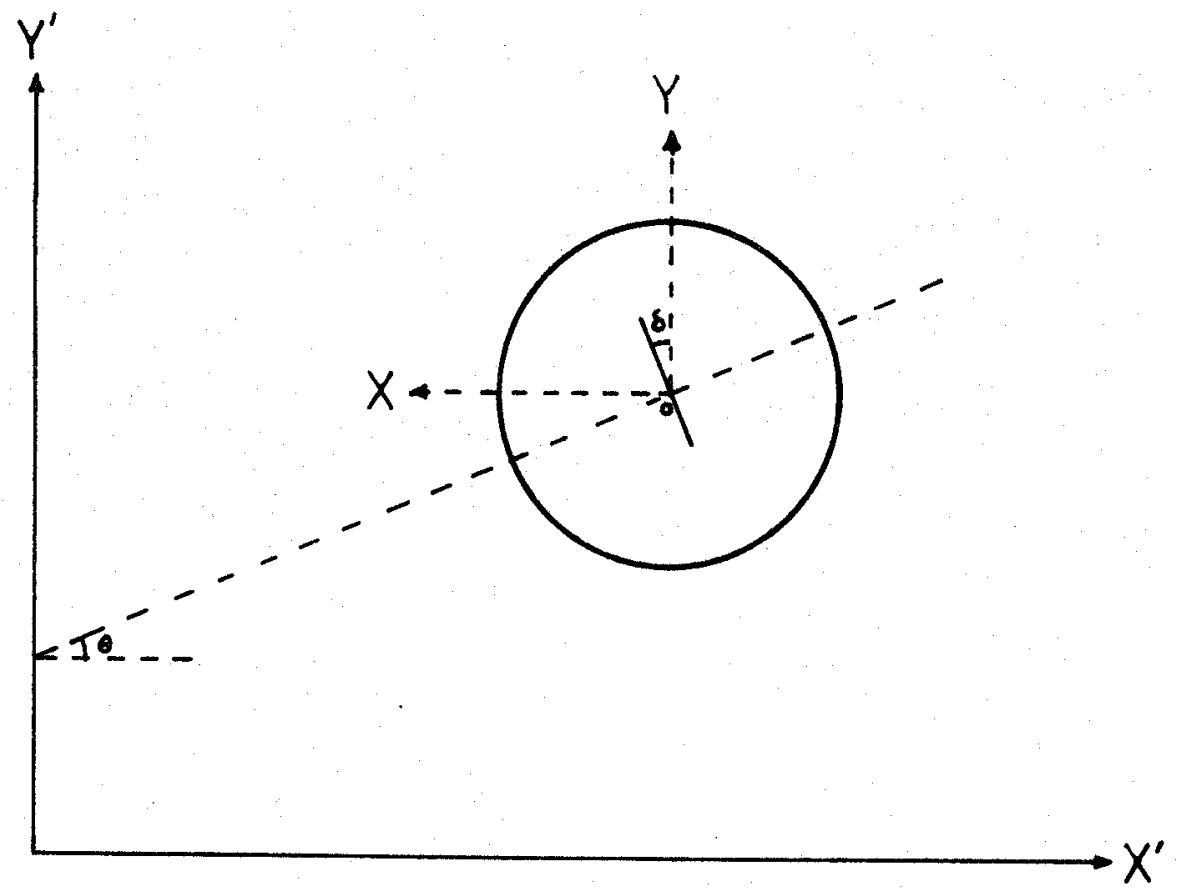

FIGURE $3.6 \mathrm{a}$ TOP VIEW OF THE DRAFTING TABLE

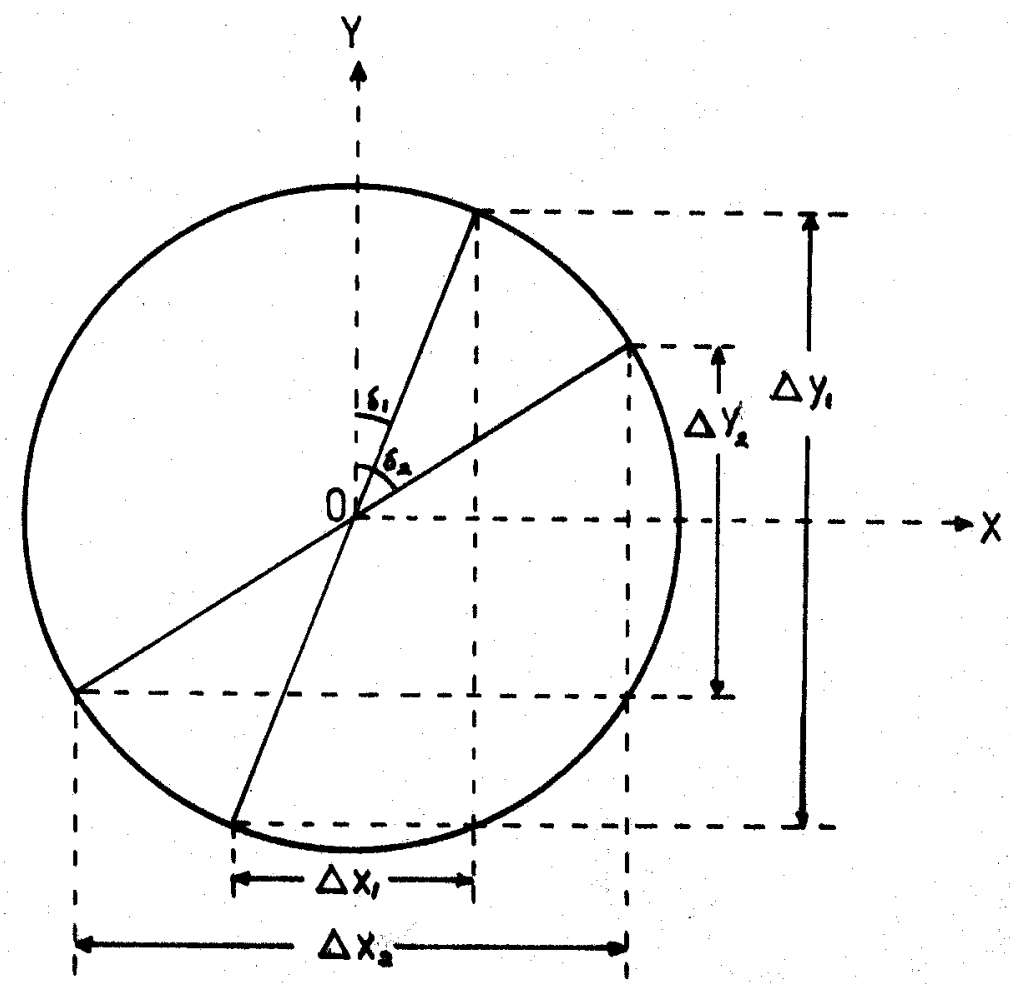

FIGURE $3.6 \mathrm{~b}$ FRONT OF THE CRT SCREEN 


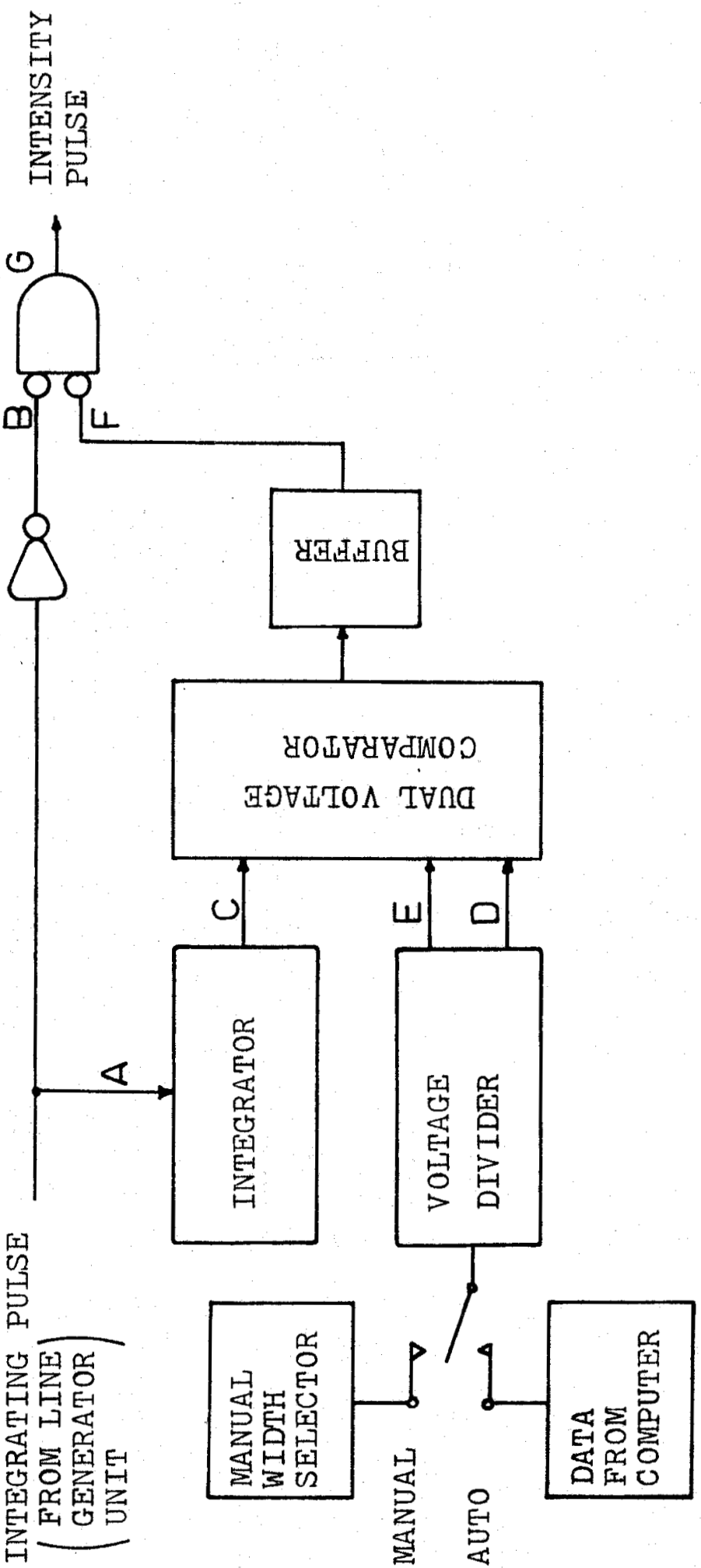

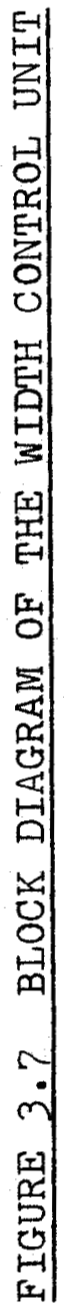


5 volts by adjusting the reference input voltage, and its duration is exactly equal to the $X$ and $Y$ ramps. This signal is then connected to a dual voltage comparator which receives other voltage levels from a programmable voltage divider. The output of the width control unit, the intensity pulse, is obtained by filtering the unwanted resetting portion from the signal coming from the dual voltage comparator. Figure 3.8 shows the output signal corresponding to the terminals located in the block diagram. From the diagram, it is noted that the lagging edge of the reference ramp in the resetting duration does not return to zero immediately. This is due to a residual charge build-up, through the onresistance of the switch on the integrating capacitor.

The block diagram of the programmable voltage divider is shown in Fig. 3.9. The output voltages from the terminals $\mathrm{E}$ and $\mathrm{D}$ are selected by changing the resistance value across these terminals. The decoder, obtaining the width input signal via the width register from the computer, selects the resistance value by closing the corresponding switch. For calibration, the width input signal can also be supplied from the manual width selector on the front panel of the CRT interface unit. Eight outputs from the decoder are available in the unit: one of them is applied to inhibit the intensity pulse in order to turn off the electron beam; the others are used for resistance selection. 


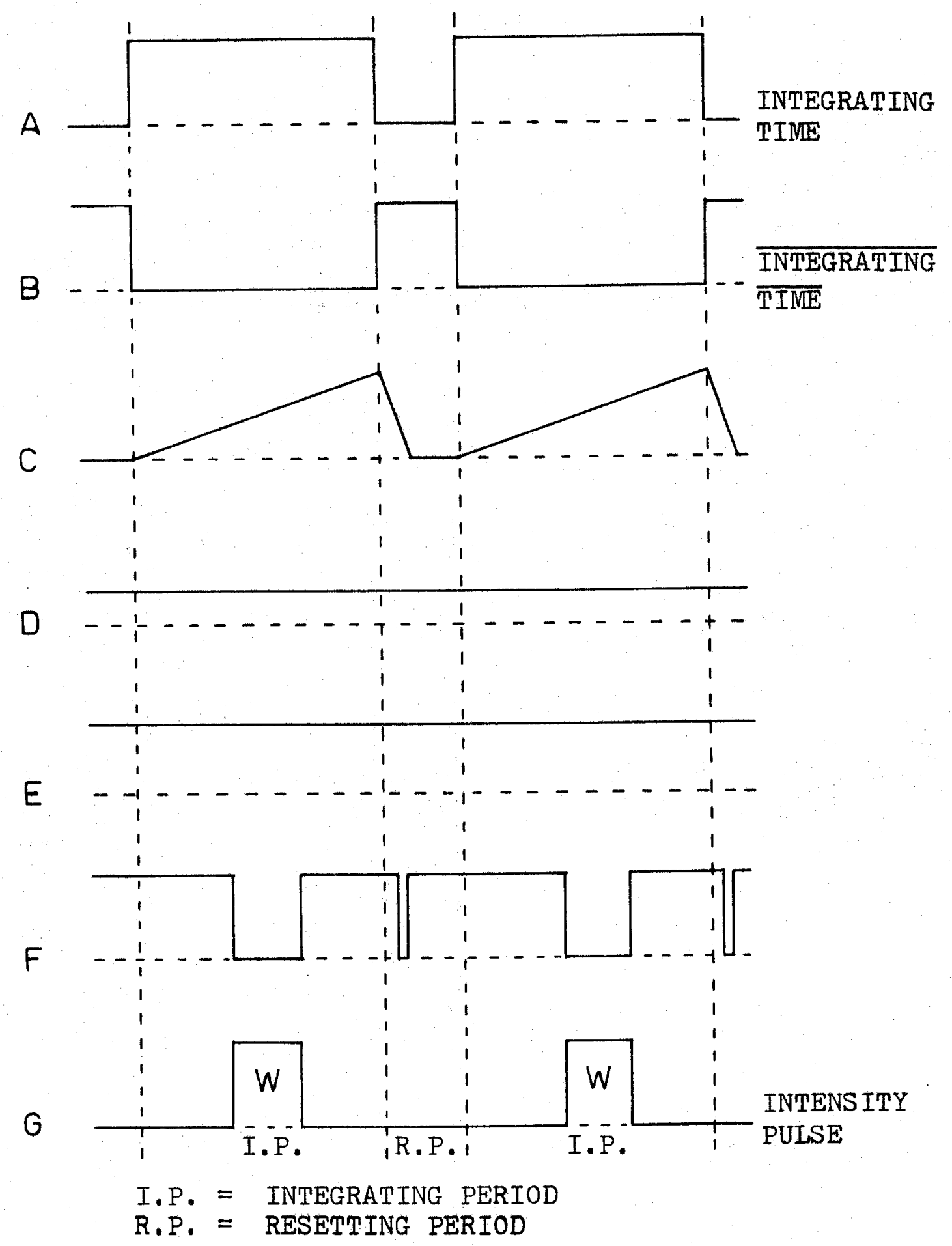

FIGURE 3.8 WAVEFORMS OF THE SIGNALS FROM THE WIDTH CONTROL UNIT 


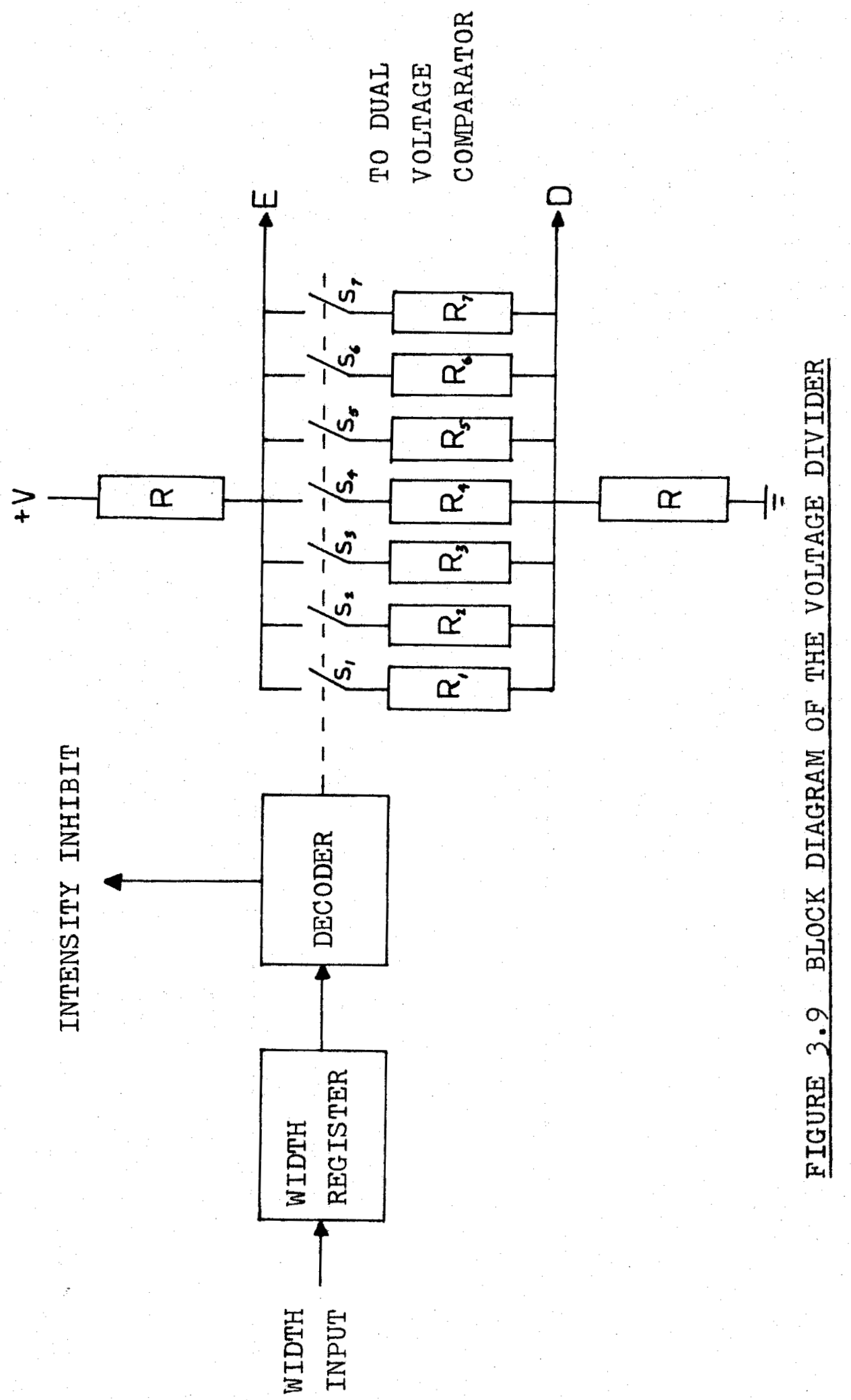


The advantages of this approach are three-fold:

a) The intensity of a scan-line is not affected by varying the length of the scan-line image. This is because the CRT beam always traces out a line of a constant length, and of a constant duration. The sweep rate of the CRT beam as well as the intensity of the line is constant so that there is no need for intensity correction as is necessary for variable length scanline generation with a conventional analog technique.

b) The visual image is only intensified at the middle section of the line generated by the line generation unit. The crooked line due to the ringing at the ends of ramps is thus avoided.

c) Only one look-up table is required to be compiled for the control of rotation if the voltage levels from the terminal $E$ and terminal $D$ are symmetrical about the center of a scan-line.

\subsubsection{Intensity control}

The exposure on a film is defined as the product of the intensity of light (CRT) seen by the film and the time the film sees the light. Whenever the speed of the drafting table varies, the optical density of the image will be changed unless the product of the intensity of light and the exposure 
time can keep constant. Usually, the intensity of a CRT is controlled by the beam current or the acceleration grid voltage. However, in both the electrostatic deflection CRT and the electromagnetic deflection CRT, the spot size of the CRT is affected by varying the beam current ${ }^{(14)}$. This is largely due to the space charge effect: the mutual repulsion of the electrons tends to spread the beam, thus increasing the spot size. In addition, excessive spot brightness produces a halo effect due to internal reflections within the screen and thus affects the quality of the line drawn on a film.

On the other hand, for a certain intensity level of a CRT, the exposure on a film can be maintained by the time parameter. A scan-line image is generated repeatedly at a rate according to the speed of drafting so that the time for which the film sees the light is constant. In other words, the faster the drafting table proceeds, the higher the frequency of the unblanking pulse sent to the $Z$-axis of a CRT. This principle was employed for the intensity control of the line drafting system.

In the Gerber 22 drafting table, the $X^{\prime}$ and $Y^{\prime}$ axes are independently driven by stepper motors. The velocity of either axis can be specified by monitoring the frequency of the pulses supplied to its stepper motor from the drafting table interface unit. For the purpose of increasing 
accuracy, the $X^{\circ} 8$ and $Y \cdot 8$ pulse trains, as previously mentioned, are employed instead of the $X '$ ' and $Y^{\prime}$ pulse trains. Figure 3.10 shows the block diagram of the intensity control unit. Both the X'8 and Y'8 pulse trains are sent to the two input channels of frequency-to-voltage-converter where analog voltages proportional to their frequencies are produced. The analog voltages are then sent to the velocity compute circuit. Using the Pythagorean theorem, the output voltage from the velocity compute circuit has an amplitude equal to the square root of the sum of the squares of the two input voltages. This voltage, which represents the total velocity of drafting, is then converted into a pulse train by the voltage-to-frequency converter. The frequency of this pulse train is thus proportional to the velocity of drafting. The pulse train is then applied to trigger the integrating timing-gate of the line generator unit. In this way, the repetition rate of generation of the scan-line image on the screen of the CRT light-head is controlled automatically by the speed of drafting, and thus controls the intensity of a scan-line.

In the intensity control unit, besides the auto mode just described, there is a manual mode for the control of intensity. A manual adjustable clock is provided for selecting the desired repetition rate of the scan-line. This is especially useful in calibrating the intensity of a CRT 


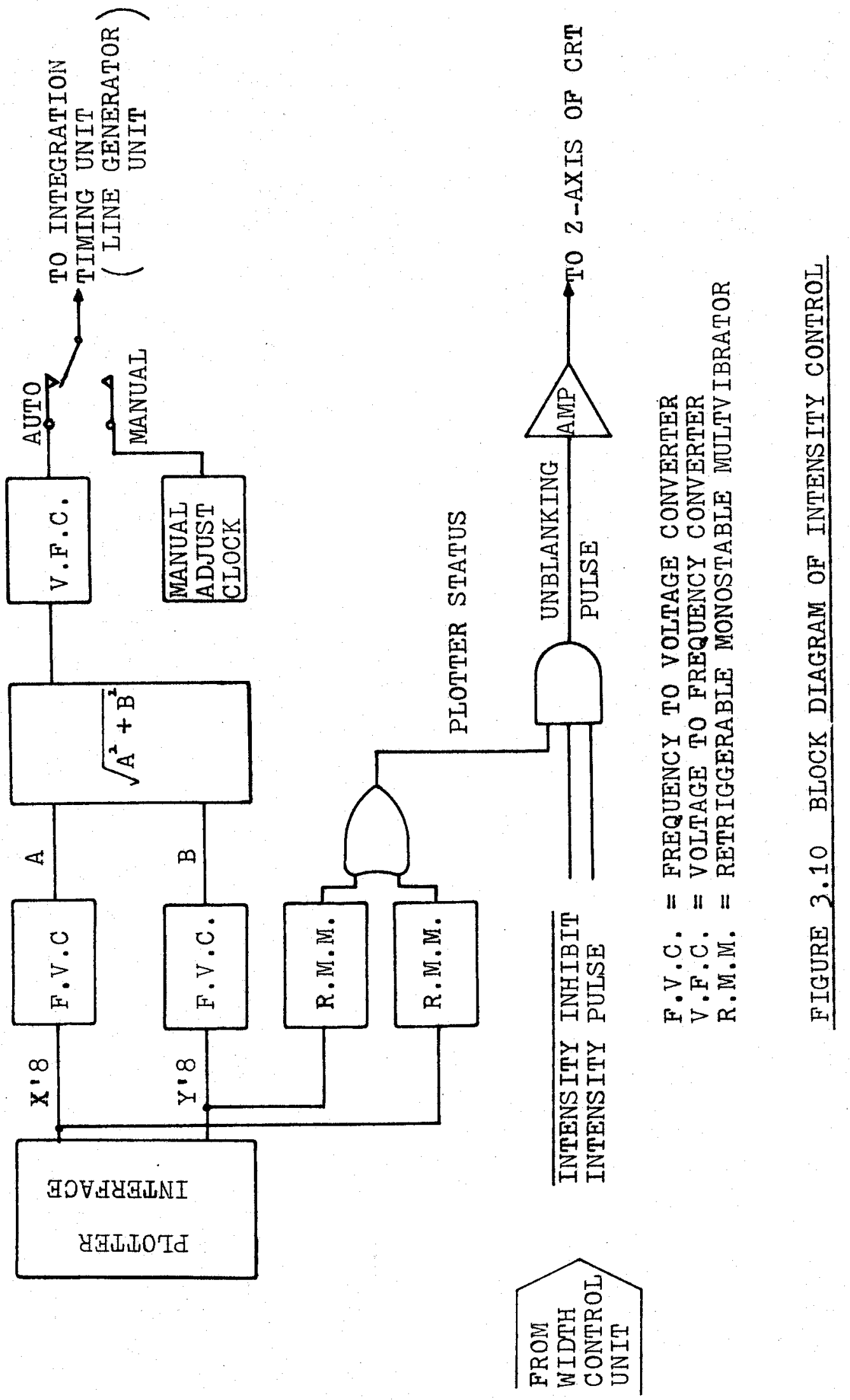


or in an exposure test.

In order to ensure that the electron beam of the light-head is completely turned off when the drafting table comes to the end of a line, or if it stops during the drafting operation, the plotter status signal from the 'or' gate is set to reflect the go/stop state of the drafting table. (See Fig. 3.10). The inputs to the 'or' gate come from two retriggerable monostable multivibrators where the logic signal will be true when a train pulse from the drafting table interface is received. Consequently, the plotter status signal will be true if, and only if, the drafting table is in movement.

other signals such as the intensity inhibit signal and the intensity pulse signal from the width control unit are connected as inputs to the 'and' gate in addition to the plotter status signal for generating an unblanking pulse. The intensity inhibit signal will be false only if the width input is at the value of zero which indicates the off-beam command. The intensity pulse signal signifies the time period for a desired length of scan-line image. Under the conditions that all the signals of the plotter status signal, the intensity inhibit signal and the intensity pulse signal are true, a visual image is traced on the screen of the light-head by sending the unblanking pulse to the 2 -axis of the CRT through a voltage amplifier. 
3.4 The input/output transfer instructions

The input/output transfer instructions (IOT) are used to initiate the operation of peripheral devices and to transfer data between peripherals and the central processor. To conserve the number of IOT'S, all the necessary information for the system operation is transferred from the accumulator (AC) of the mini-computer into the control unit as a command word. Figure 3.11 lists the IOT's that are to be used to allow the PDP8/e mini-computer to control automatically the functions in the line drafting system.

\subsection{Sequence of operation}

Flowchart 3.1 indicates, in a simplified manner, the normal sequence of events for the data search and linedrafting operation as directed by the line-drafting program. For standard line-drafting application, the linedrafting program performs the disc directory look-up function to find which data file or files hold the desired data. In cartography, digitized data can be stored either in an incremental or in an absolute format. However, in this particular line drafting system, only the incremental format is acceptable, so that data in absolute format has to be converted into incremental format during the line drafting operation. Furthermore, in the application of cartographic line drafting, 'forward-look-linear' interpolation is applied 
INSTRUCTION

FUNCTION

6141 Load XB buffer register from PDP8/e accumulator. The accumulator is not automatically cleared by this operation. (Load initial position data of $x$ of the scan-line).

6142 Load YB buffer register from PDP8/e accumulator. The accumulator is not automatically cleared by this operation. (Load initial position data of $y$ of the scan-line).

6144 Load width buffer register from PDP8/e accumulator. The accumulator is not automatically cleared by this operation.

6132 Load XA buffer register from PDP8/e accumulator. The accumulator is not automatically cleared by this operation. (Load $\mathrm{x}$ data of the scan-line).

6134 Load YA buffer register from PDP8/e accumulator. The accumulator is not automatically cleared by this operation. (Load y data of the scan-line).

6331 Skip next sequential program instruction if the control busy flag is raised.

6332 Skip next sequential program instruction if the plot busy flag is set.

6333 Skip next sequential program instruction if the normalization error flag is set.

6321 Clear all registers and flags in the controller.

6322 Load the most significant 12 bits of $X$ ' from PDP8/e accumulator. The accumulator is automatically cleared by this operation.

6323 Load the least significant 12 bits of $X^{\prime}$ from PDP8/e accumulator and then clear the accumulator.

6324 Load the most significant 12 bits of $Y$ from PDP8/e accumulator and then clear the accumulator.

6325 Load the least significant 12 bits of $Y^{\prime}$ from PDP8/e accumulator and then clear the accumulator.

6326 Load status register from PDP8/e accumulator and then clear the accumulator.

6327 Plot go (commands the control logic to proceed with pre-plot processing). If the plot busy flag is not up, the controller will proceed with the plot. If plot busy flag is set, the controller will wait for it to reset, then proceed with the plot.

FIGURE 3.11 I.O.T. FOR THE SYSTEM 
Enter from

line-drafting program

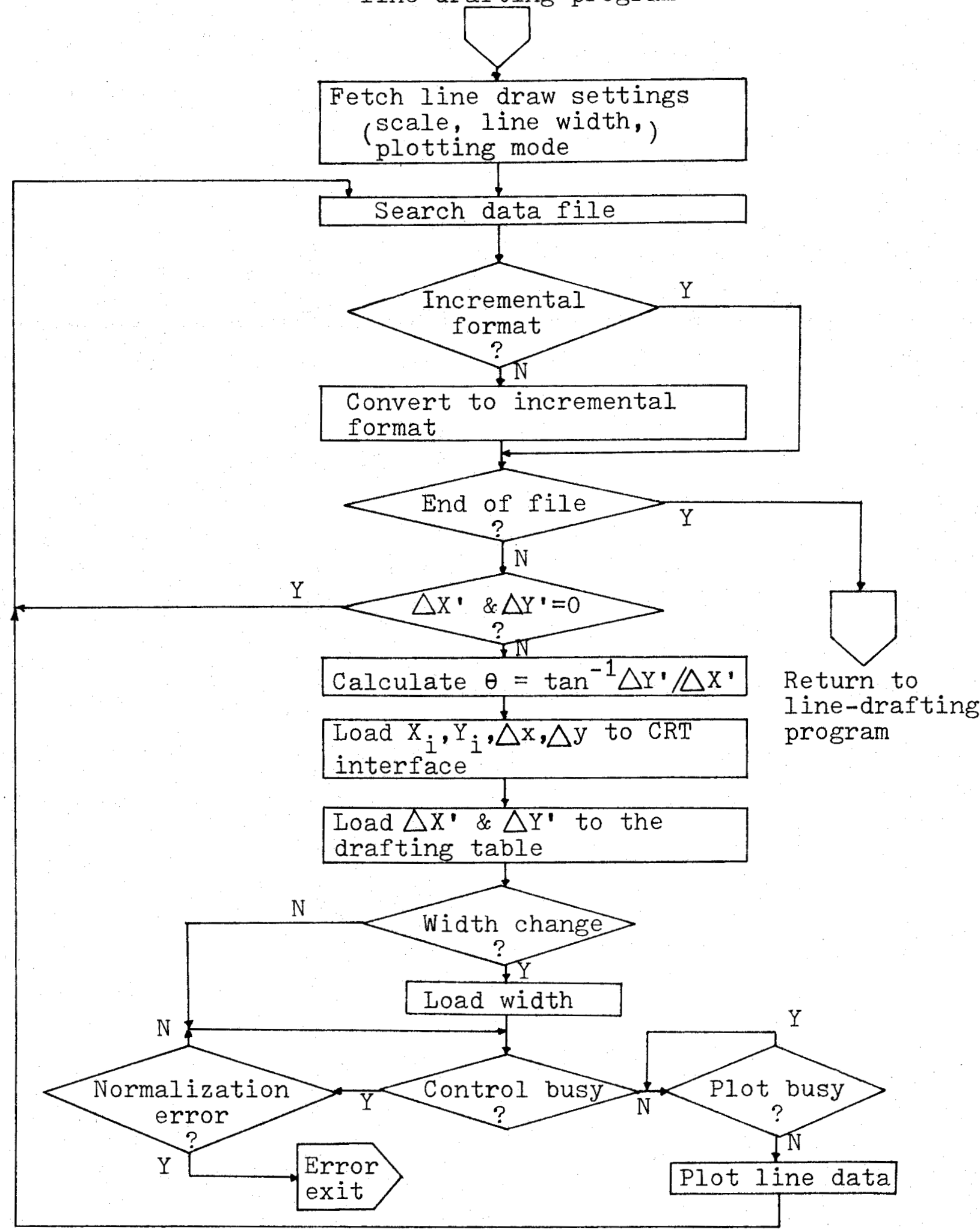

FLOWCHART 3.1 LINE-DRAFTING 
for the purpose of generating a smoother line output. Further details of this method are given in Appendix (B).

The line width and the beam on/off command are both controlled by the selection of the width input to the width control unit. A width of zero is the off command. It should also be noted that a normalization error will occur in the drafting unit interface whenever both the incremental data, $\Delta X^{\prime}$ and $\Delta Y^{\prime}$, are zero. This error is eliminated by software detection so that the zero incremental data will not be loaded to the drafting unit interface.

Once the drafting settings such as the scale of the map, the width of the cartographic line and the drafting mode are given, no human intervention is required for the system during the line drafting operation.

\subsection{Samples of line drafting} 3.6 .1 General

This section discusses the performance of the CRT light-head prototype system and associated hardware presented in an earlier section. The purpose of testing the prototype system was to verify the feasibility of the proposed automatic drafting system and to identify all variables that could have a significant effect on the quality and accuracy of results.

Much emphasis was placed on evaluating the system 
from the cartographic user's point of view; that is, its ability to draft on film to a very high quality. However, due only to the limitations in quality of the CRT, as well as to its mounting, the output from this experimental prototype system was far below the quality which would be considered adequate for a production unit.

All of the line drafting tests were conducted with a rectangular shaped light spot using the GAF ECONOLINE P4 film. Under the magnification of the optical lens (approximately 0.15$)$, the width of a scan-line image focussed onto the film is equal to $0.09 \mathrm{~mm}$ and the length of it varies from $0.1 \mathrm{~mm}$ to $0.7 \mathrm{~mm}$, dependent on the width control unit. The former value is governed by the spot size of the CRT lighthead (approximate $0.6 \mathrm{~mm}$ ) at a certain grid voltage, while the latter is controlled by the gain of the deflection amplifiers.

Three types of tests were performed in line drafting. Firstly, an exposure test was conducted to determine the maximum drafting speed as well as the scan repetition rate. Secondly, graphical data generated mathematically from the computer was applied for testing the dynamic image rotation and the line width generation. Thirdly, digitized cartographic data were employed directly for map generation using forward-look linear interpolation. 


\subsubsection{Drafting on film}

\subsubsection{Exposure test}

The first parameter that must be considered when a light-head is employed for line drafting is the relationship between the intensity of the light-head and the speed of drafting. For a certain drafting material, the intensity control must vary the intensity of the projected image proportionally to its velocity to maintian constant exposure conditions. In this system, it is accomplished by varying the frequency of the scan repetition.

The maximum drafting speed of the system was determined by drawing a series of lines ( $0.3 \mathrm{~mm}$ in width) at selected frequencies of $45 \mathrm{~K} \mathrm{~Hz}$ and $40 \mathrm{~K} \mathrm{~Hz}$. The exposure time of each line was varied by manually adjusting the speed of the drafting table. Figure 3F.1a shows a sample of the results. It should be noted that the $45 \mathrm{~K} \mathrm{~Hz}$ was the cutoff frequency of the line generation unit and, therefore, the $40 \mathrm{~K} \mathrm{~Hz}$ was selected as the maximum scan repetition rate of the projected image. From that figure, the maximum drafting speed of the prototype system was defined to be $1.22 \mathrm{~cm} / \mathrm{sec}$ by comparing the optical density of the film output.

In order to determine the function of the quality of the line in terms of the repetition scanning rate and the velocity of drafting, a series of lines was drawn at selected speeds from $0.04 \mathrm{~cm} / \mathrm{sec}$ to $1.22 \mathrm{~cm} / \mathrm{sec}$ (Figure $3 \mathrm{~F} .1 \mathrm{~b}$ shows the samples of $1.04 \mathrm{~cm} / \mathrm{sec}$ and $1.22 \mathrm{~cm} / \mathrm{sec}$ ) while manually 


\begin{tabular}{|c|c|c|c|}
\hline \multicolumn{2}{|c|}{ SPEED } & FREQUENCY $=45 \mathrm{~K} \mathrm{~Hz}$ & FREQUENCY $=40 \mathrm{~K} \mathrm{~Hz}$ \\
\hline $\mathrm{cm} / \mathrm{sec}$ & in/sec & & - \\
1.22 & 0.48 & - & - \\
1.45 & 0.57 & - & - \\
1.83 & 0.72 & - & - \\
2.08 & 0.82 & - & \\
2.44 & 0.96 & - & \\
2.92 & 1.15 & & \\
3.66 & 1.44 & & \\
4.88 & 1.92 & & \\
\hline
\end{tabular}

FIGURE 3F.1a

\begin{tabular}{|c|c|c|}
\hline $\begin{array}{c}\text { FREQUENCY } \\
\mathrm{K} \mathrm{Hz}\end{array}$ & $\begin{array}{c}\text { SPEED }=1.04 \mathrm{~cm} / \mathrm{sec} \\
(0.41 \mathrm{in} / \mathrm{sec})\end{array}$ & $\begin{array}{c}\text { SPEED }=1.22 \mathrm{~cm} / \mathrm{sec} \\
(0.48 \mathrm{in} / \mathrm{sec})\end{array}$ \\
\hline 45 & - & - \\
40 & - & - \\
35 & - & - \\
30 & - & - \\
25 & - & - \\
20 & - & - \\
15 & - & - \\
10 & - & - \\
8 & - & - \\
\hline
\end{tabular}

FIGURE $3 \mathrm{~F} .1 \mathrm{~b}$

FIGURE 3F.1 SAMPLE OF EXPOSURE TEST 
adjusting the repetition frequency of the scan-line. Ideally this relation should have varied linearly with the drafting speed. However, the high contrast nature of the film made it difficult to obtain valid results from the test. Any slight variation of illumination across the width of the line produced a significant change in density. Hence, some other experiments with lower drafting speeds were conducted separately. The results showed that the function is not linear, especially at lower velocities (less than $0.2 \mathrm{~cm} / \mathrm{sec}$ ). The lower velocities required a higher frequency rate than would have been expected. It is believed that this nonlinearity is caused by a phenomenon known in photography as the intermittency effect. An intermittent exposure is not equal to a continous exposure for the same time but differs by an amount which depends upon the rate of intermittency and the intensity level of exposure.

The non-linear characteristics of the intensity control did not adversely affect the quality of lines produced for the range of drafting speeds used when the operating point of the control is set high enough to ensure proper line exposure for low speeds. It was found that a varying frequency range from $30 \mathrm{~K} \mathrm{~Hz}$ to $40 \mathrm{~K} \mathrm{~Hz}$, when at upper drafting speed (greater than $0.2 \mathrm{~cm} / \mathrm{sec}$ ) of the drafting table, was appropriate for the intensity or scan repetition frequency control. When at lower drafting speeds, a constant frequency 
of $30 \mathrm{~K} \mathrm{~Hz}$ produced acceptable results. The excess amount of intensity that is present at very slow speeds is allowable because of the large exposure latitude of the photographic film used.

3.6.2.2 'Mathematically based' line data drafting

'Mathematically based' Iine data for a sine-wave were generated by the computer and applied for line drafting in the system. A sine-wave was chosen mainly because it provided an excellent check on the dynamic image rotation and width tolerances of a line drawn at a diversity of angles. The width tolerances were determined by the stability and accuracy of the rotation and width control units. The most significant source of error resided in the drift of the rotational center of the projected image during curved line tracing. This was caused partly be the lag of the $Z$ pulse received from the intensity control unit to the CRT light-head through the cable carrying signals between them, and partly by an irregularity in the programmable voltage divider of the width control unit. This error was eliminated by a compensating circuit.

Figure $3 \mathrm{~F} .2$ and Figure $3 \mathrm{~F} .3$ show the quality that can be obtained at different line widths with the experimental CRT light-head and the Gerber drafting table. From these, the width of the sine-waves shown are $0.3 \mathrm{~mm} \pm 0.01 \mathrm{~mm}$ in 
Fig. 3F.2, and $0.5 \mathrm{~mm} \pm 0.01 \mathrm{~mm}$ in Fig. $3 \mathrm{~F} .3$ respectively. It will be noted that all the points along the sine-wave are drawn smoothly except when the peaks of the line are reached. It is believed that this graphical irregularity was caused by a backlash in the driven gear on reversal of direction of the drafting unit which was a fault present at the time of the tests.

3.6.2.3 Digitized cartographic line data drafting

The results obtained by the point-to-point linear interpolation for a map generation with cartographic data which are digitized from maps were not as good as those obtained with 'mathematically based' data. The reason is that the data of cartography such as a shoreline usually has many abrupt changes in its direction. The dynamic image is unable to follow these abrupt changes unless a waiting loop is generated in software programming. However, the waiting 100p appreciably decreases the drafting speed. In addition, as a result of the backlash in the driven gear of the drafting unit, the edges of the line became jagged and there is noticeable drift at the turning points of a line.

The smoothness requirement of cartography was met by using a four-point look-ahead linear interpolation. Figure 3F.4 shows a sample plot in a shoreline arafting. In the figure, the width of the shoreline shown is $0.3 \mathrm{~mm} \pm 0.015 \mathrm{~mm}$. 
3.6.3 Cause of irreqularities

In general, the film produced by the experimental light-head system indicates that the system is working properly in line drafting within the operating speed of the drafting table. In addition to the deviations such as the drift in dynamic image rotation, the intermittency effect and the backlash of the drafting unit have to be taken into account when the performance of the system is considered. There was some drift of the d.c. levels caused mainly by the temperature sensitivity of the operational amplifiers used. It affected both the stability and accuracy of the hardware circuit and thus the quality of line obtained. This could be minimized by employing a temperature compensation circuit in any future hardware design. The gain of some of the components was frequency dependent. This caused some noticeable errors when the system was operated at extreme frequencies. These errors could be reduced by using large bandwidth components with better cutoff frequencies. The film processing conditions such as temperature, development time and humidity represent other factors that affected the width and density of lines (15)(4).

Furthermore, the optical aberration of the lens, the mechanical tolerance of the light-head mounting and the flatness of the drafting table directly affected the performance of the system. 


\section{AREA FILL}

\subsection{General}

One of the advantages of a CRT light-head is that it can generate any shape of image as desired. In cartography, the generation of area colour overlays is achieved by manually removing areas of 'peel-coat' sheet material. This process is extremely tedious and time consuming. In this chapter, an 'area-fill' program is introduced to alleviate this problem by using the CRT light-head. This program automatically analyses the data around the perimeter of a polygon (i.e. a lake, an island or a bounded area) and fills the inside areas of the polygon according to the 'fill-in' pattern selected by the user.

In addition to the block-fill pattern, where the entire defined area undergoes solid fill, six 'fill-in' shaded patterns can be obtained by changing the slope of the filling lines, or their spacing. Different 'fill-in' patterns can be employed for the separation of areas or countries in a map presentation. In practice, four different 'fill-in' patterns are sufficient to prevent the same pattern appearing on both sides of any boundary on any map on a sphere (or consequently on any planar projection of such a map) (16).

In order to obtain maximum flexibility with minimum positional error and a relative uniform intensity over the 
entire figure, a digital technique for line generation is used. In other words, the 'area-fill' program is carried out by the character generation unit instead of the line generation unit as in the proto-type automatic drafting system. This unit was presented by Wilkie ${ }^{(5)}$. Furthermore, a visual check of the performance of the 'area-fill' program is allowable by displaying the filled area on the Tektronix 611 storage display.

4.2 Programming

4.2.1. Input parameters

4.2.1.1 Window

'Window' is defined as a rectangular area within the screen of a CRT which is available for area fill. (See Fig. 4.1). The dimensions of the window were designated as the height of the window (Wh) and the width of the window (Ww).

4.2.1.2 Fill-in pattern code

Seven codes are available for different pattern fillings. The lines from which they are made are signified by two variables, namely the slope of the lines (M) and the spacing between them $(S)$. (The spacing is in terms of the addressable line spacing of a CRT.) The patterns are listed according to their code numbers in the following table. 


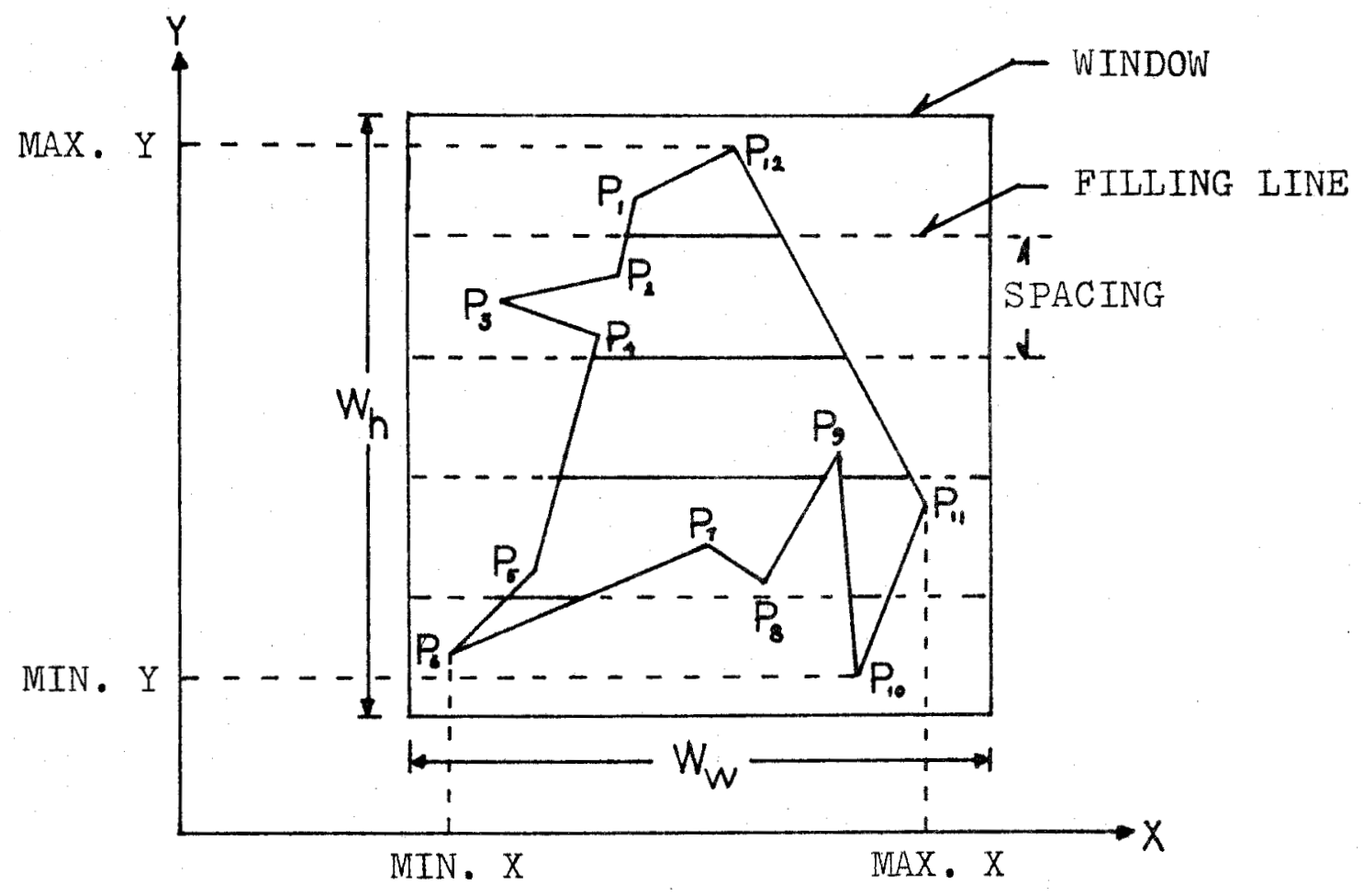

FIGURE 4.1a FILL-IN PARAMETERS

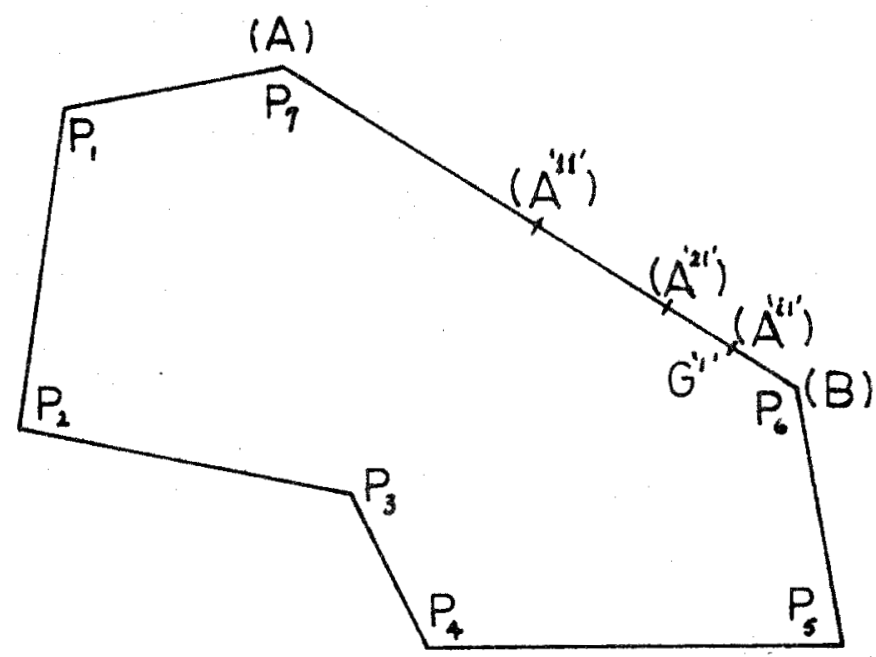

FIGURE $4.1 \mathrm{~b}$ MID-POINT APPROACHING 


\begin{tabular}{|c|c|c|c|}
\hline CODE & M & $S$ & REMARK \\
\hline 1 & 0 & $10_{8}$ & \\
\hline 2 & 1 & 148 & \\
\hline 3 & -1 & 148 & \\
\hline 4 & $\infty$ & $10_{8}$ & \\
\hline 5 & & & combine code 1 and code 4 \\
\hline 6 & & & combine code 2 and code 3 \\
\hline 7 & 0 & 1 & block fill \\
\hline
\end{tabular}

4.2.1.3 Data format

Only absolute coordinate format is at present acceptable for the 'fill-in' algorithm. For example, a polygon with $n$ sides has $n$ vertices, $P_{1}, P_{2}, P_{3} \ldots P_{n}$ (see Fig. 4.1a). The coordinates $\left(x_{i}, y_{i}\right)$ of all vertices of the polygon have to be stored in the memory of the PDP8/e mini-computer sequentially, either in a clockwise or counterclockwise order. This format of data was digitized and compiled in a DEC OS/8 structure file through the cartographic system developed at the University of Saskatchewan.

4.2.2 Processing a filling-line

The equation of a filling line is defined as $Y_{i}=M_{i}+b+i S$

Where $Y$ is the $Y$ coordinate

$X$ is the $X$ coordinate

$i$ is the number of the filling-line

$\mathbb{M}$ is the slope of the filling-line

$S$ is the spacing between filling-lines 
$b$ is the $Y$ coordinate that the starting filling-line meets the $Y$ axis. It is determined by the size of window and the slope of the filling-line.

Assuming that the polygon to be filled is within the allowed window, all filling-lines inside the polygon have to be detected for the fill-in purpose. There are two fundamental parts of the processing which determine whether a line meets the above condition, namely the intersection point computation and the point-in detection.

4.2.2.1 Intersection point computation

Mathematical calculation for the intersection of two given straight lines is relatively complicated and tedious even using machine language for programming. Therefore, instead of calculating the intersection point of a fillingline and the polygon, all the points along the perimeter of the polygon are substituted in turn into the equation of a filling-line. A zero result indicates that the coordinate substituted is an intersection point of the boundary and this filling-line. In this way, both the coordinates and the number of intersection points are stored in the computer.

The coordinates of points along the perimeter are generated by the mid-point approaching method. For example, two adjacent vertices of a polygon are considered as two end points of a straight line. (See Fig. 4.1b point $A$ and point $B)$. In Fig. $4.1 \mathrm{~b}$, point $G^{\prime}{ }^{\prime}$ ' is generated by repeat- 
edly bisecting the segment $A{ }^{\prime i 1} B$ until the length of $A{ }^{\prime i 1} B$ is less than or equal to $\sqrt{2}$ times the grid spacing of a CRT. As a result, point $G^{\prime}{ }^{\prime}$ takes place of the end point $B$, and the new point $G^{\prime} j$ ' which is obtained in the same way is calculated in turn until it reaches the end point A. In this manner, the ' $G$ ' points move toward the end point $A$ along the mid-point locus between $A$ and $B$. The mid-point coordinates can be obtained quickly by shifting binary numbers, and this method ensures that all the points lie on a straight line within the tolerance of the distance between the discrete grids of a CRT. This method is also used for the line generation in area filling.

4.2.2.2 Point-in detection

If the number of the intersection points obtained from the above processing is equal to or greater than two, the coordinates of the intersection points are rearranged and restored in an increasing $X$ or $Y$ order according to the slope of the filling-line. After that, the line segments between these intersection points are checked separately to see if they lie inside or outside the polygon. If they are inside of the polygon, they will be filled by intensifying the light output on this portion of the line.

Except for the terminal points of the line segment which will lie on the perimeter of a polygon, all other points on a line segment are either inside or outside the 
bounded area. To examine if a given line segment lies inside or outside the polygon, it is sufficient to check if the mid-point of this line segment is inside or outside the polygon. The mid-point is selected as it is simply generated. A line through the mid-point and parallel to the $Y$ axis is constructed and the number of points of the polygon which intersect with the line are counted. If the number is odd, the line segment to be detected is inside the polygon. The only ambiguity with this detection occurs when a vertex of the polygon lies directly on the line parallel to the $Y$ axis. In this condition, the two adjacent vertices on the polygon are considered. If these two vertices are on different sides of the line, then one intersection is counted; otherwise two. In the case that one of the adjacent vertices also lies on the line, further adjacent vertices have to be considered in turn.

4.2.3 Example of area fill

Fig. 4.2 illustrates the method of processing. The polygon $\mathrm{P}_{1} \mathrm{P}_{2} \mathrm{P}_{3} \ldots \mathrm{P}_{15}$ is inside the area fill window. The filling-line $i$ meets the perimeter at two points, $a_{i}$ and $b_{i}$ (in an increasing $x$ order). The line through the midpoint $m_{i 1}$ of the line segment $a_{i} b_{i}$ parallel to the $Y$ axis meets the perimeter once at point $I_{11} \cdot$ This is an odd number (1) of intersections, and thus it indicates that the line segment $a_{i} b_{i}$ is inside the polygon. 


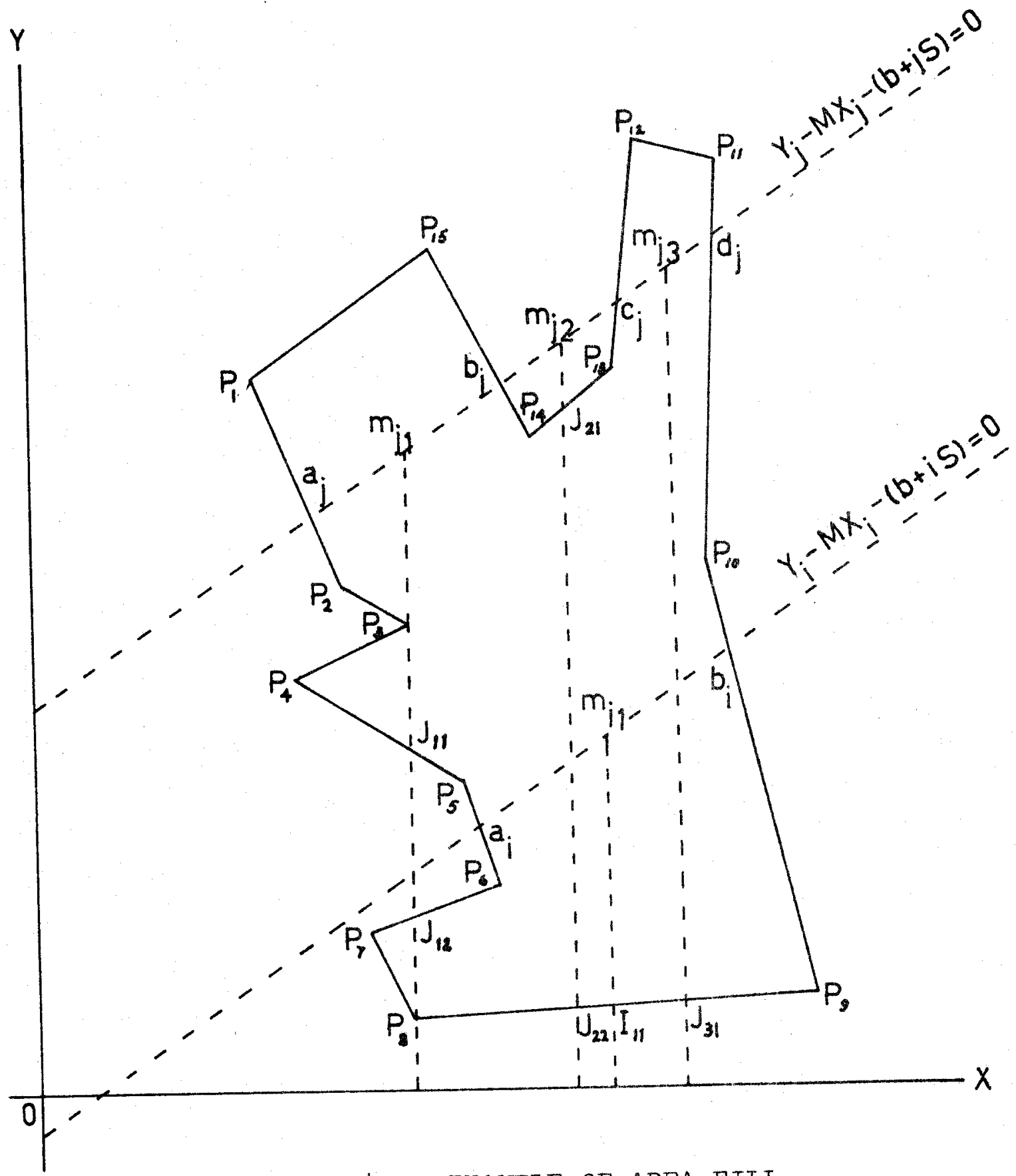

FIGURE 4.2 EXAMPLE OF AREA FILL 
The filling-line $j$ meets the perimeter at point $a_{j}, b_{j}, c_{j}$ and $d_{j}$. The Iine through $m_{j 1}$ cuts the perimeter four times at $P_{3}, J_{11}, J_{12}$ and $P_{8}$. However, since $P_{2}$ and $\mathrm{P}_{4}$ are on the same side of the line, two intersections are counted. Point $P_{7}$ and $P_{9}$ are on different sides, and thus one intersection is counted. An odd number (5) of intersections is recorded in total which thus indicates that the line segment $a_{j} b_{j}$ is inside the polygon. As for the line segment $b_{j} c_{j}$, an even number (2) is recorded so that it is outside the polygon. For the line segment $c_{j} d_{j}$, an odd number (1) is obtained so that it is inside the polygon. Flowchart 4.1 shows the area-fill subroutine.

4.2.4 Application

Up to now, it is assumed that the polygon to be filled can be accomodated within the allowable area (window) of the CRT. However, when it exceeds the dimension of the window, the polygon has to be divided into sub-polygons so that each of them can be handled within the available area of the Iight-head. This check can be performed quickly if, for each polygon, min.x, min.y, max.x and $\max \cdot y$ values of the coordinates of all its vertices are computed and stored. These minimum and maximum values can be viewed and defined as a rectangle which surrounds the polygon. (See Fig. 4.1a). Comparing that rectangle with the window quickly indicates whether further division is required. 
Enter from

area-fill program

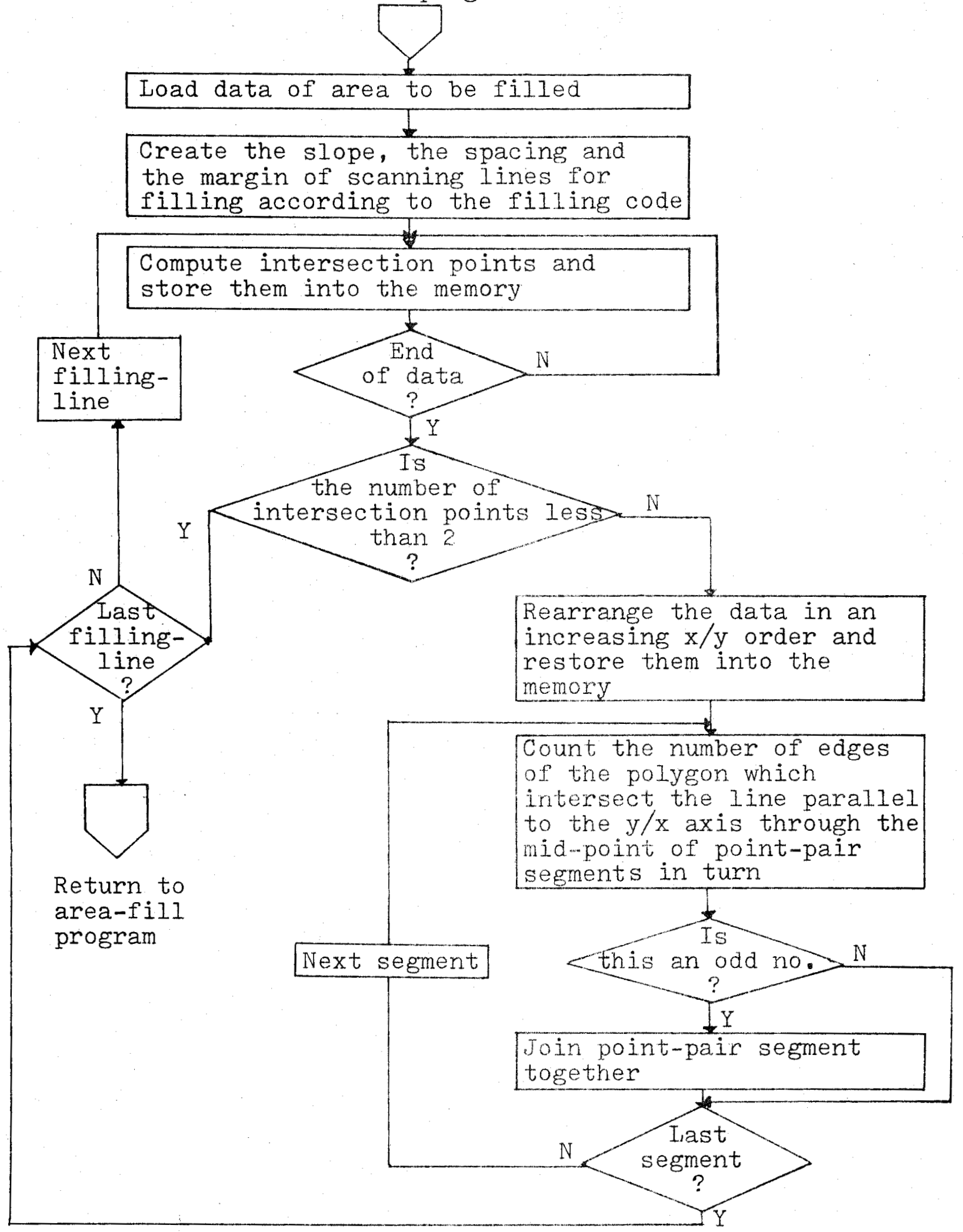

FLOWCHART 4.1 AREA-FILL SUBROUTINE 
The flow chart of the area-fill program is shown in Flowchart 4.2. The program first computes the minimum and maximum values of the polygon and compares them to the dimension of the window. If the dimension of the polygon exceeds that of the window, the program then decides the number of sub-polygons necessary. For each of these subpolygons, new area-fill data is compiled in the memory of the computer. The whole area of a polygon will be filled section by section by moving the Iight-head to each subpolygon in turn. (See Fig. 3.5a and Fig. 3.5b).

\subsection{Samples of work}

The photographs, shown from Fig. 4.3 to Fig. 4.6, were taken directly from the Tektronix 611 storage display. A one-to-one print of the CRT light-head film output is shown in Fig. 4F.1 to illustrete the actual performance of the system.

From Fig. 4F.1a, it can be noted that there is a large deviation in width of the boundary line of the areas. (In fact, the width and the density of a Iine generated by a digital technique varies according to its slope). The major error in width, however, is due to the curvilinear distortions of the optical lens. Since a 3-inch square area on the screen of the CRT is focussed down onto the film, the rays pass through the lens at a large angle to the 


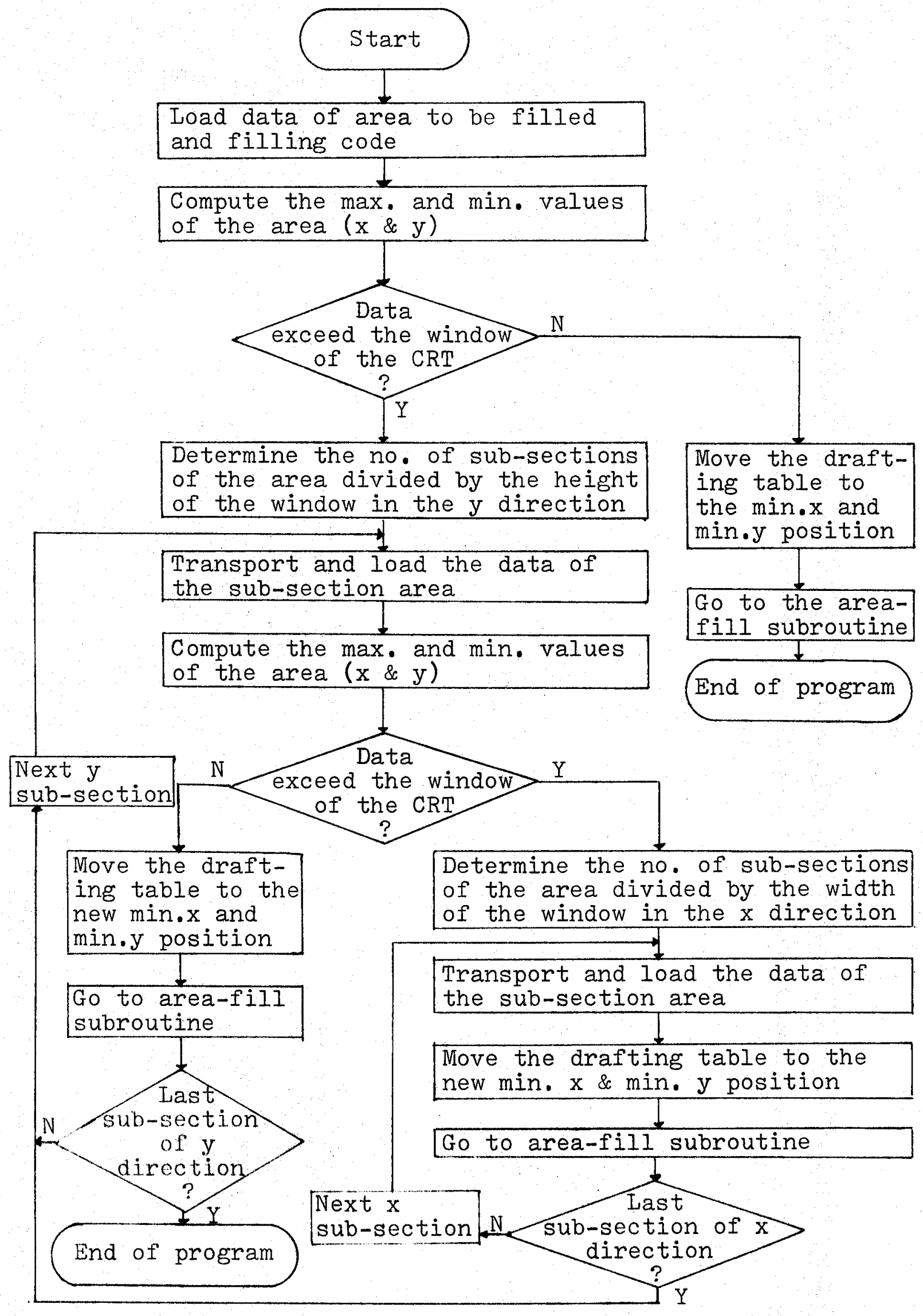

FLOWCHART 4.2 AREA-FILI PROGRAM 


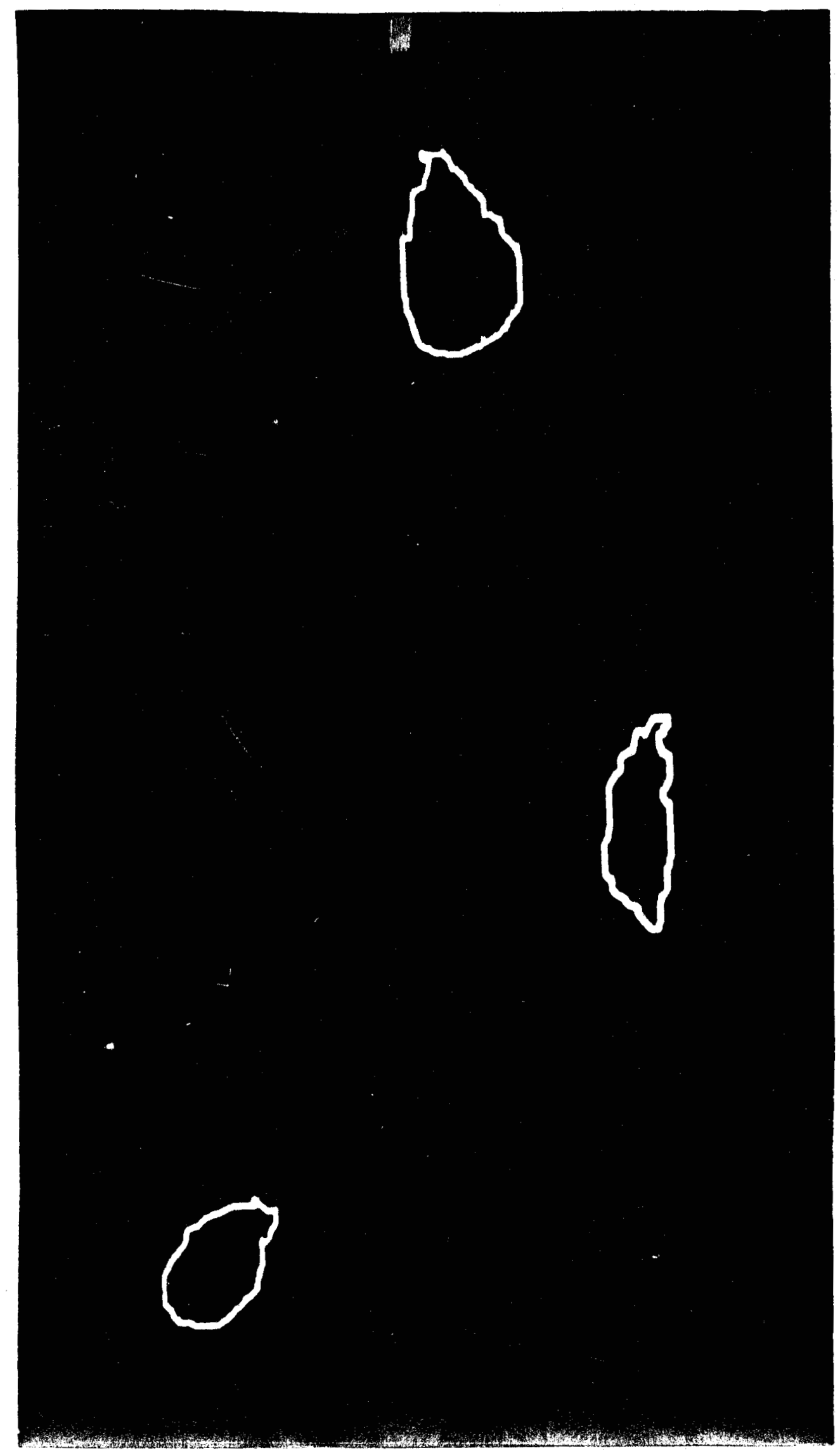

FIGURE 4.3 BOUNDARY OF AREA-FILL (611 display) 


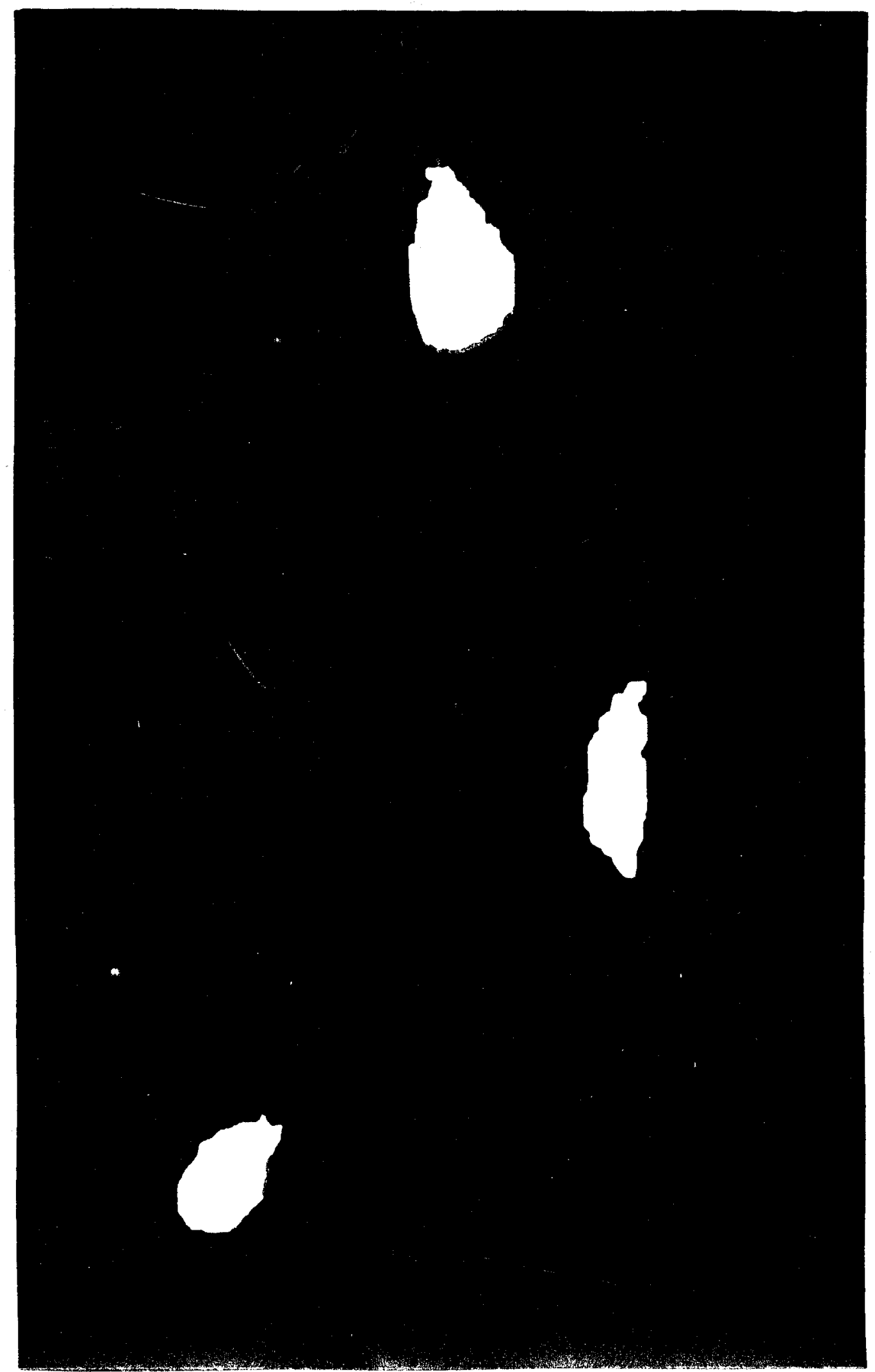

FIGURE 4.4 SAMPLE OF BLOCK-FILL 


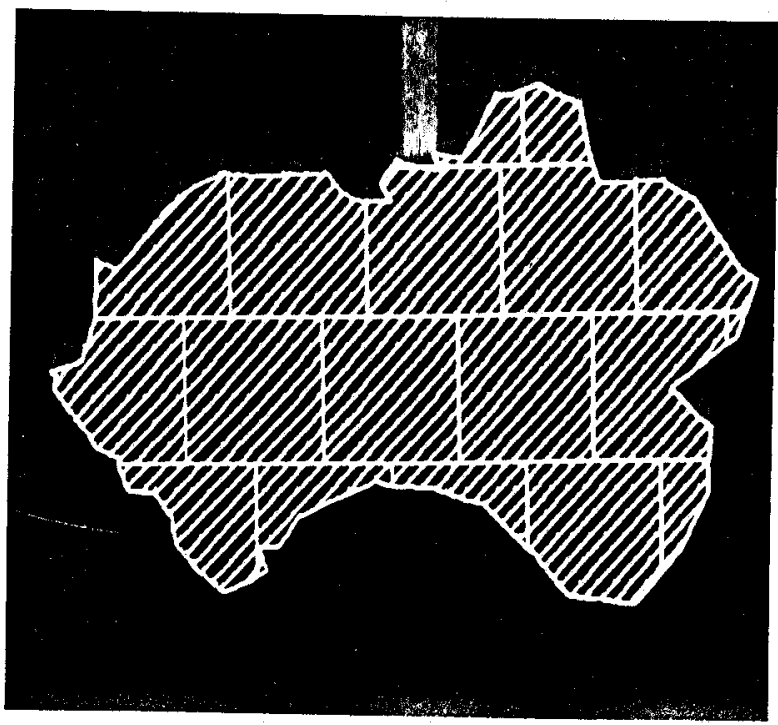

\section{FIGURE $\quad 3.5 \mathrm{a}$}

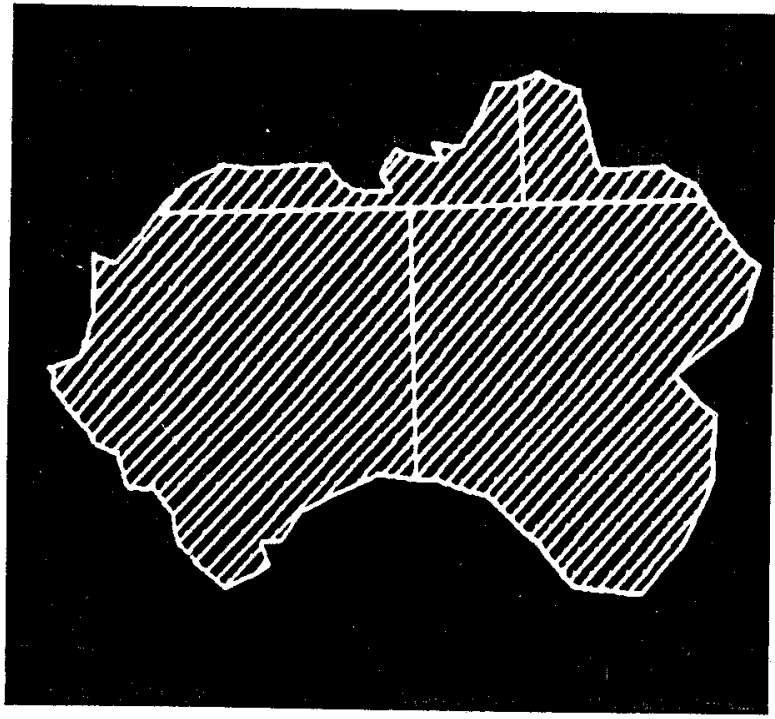

FIGURE $3.5 \mathrm{~b}$

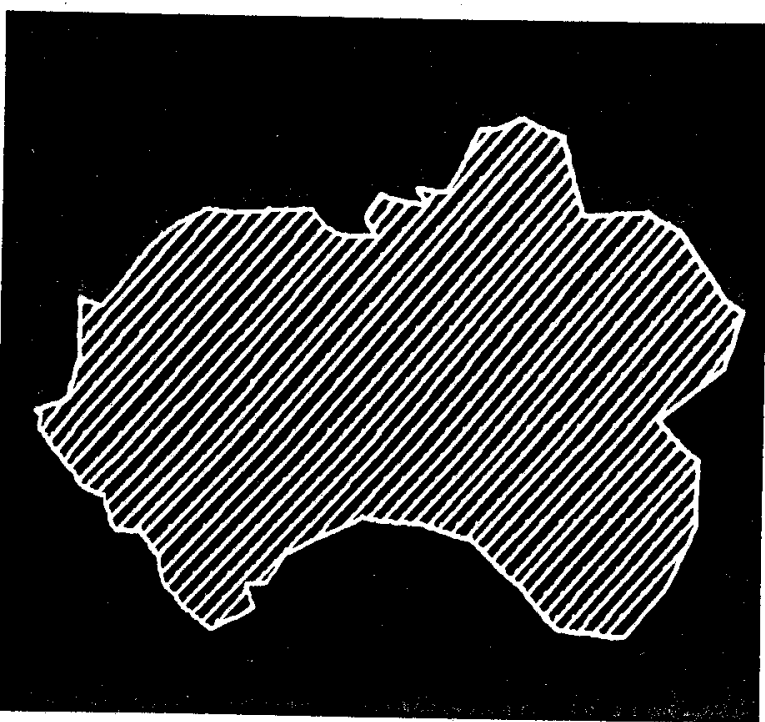

FIGURE $\quad 3.5 \mathrm{c}$

FIGURE 4.5 SAMPLE OF AREA-FILL BY VARYING THE DINENSION OF THE WINDOW 

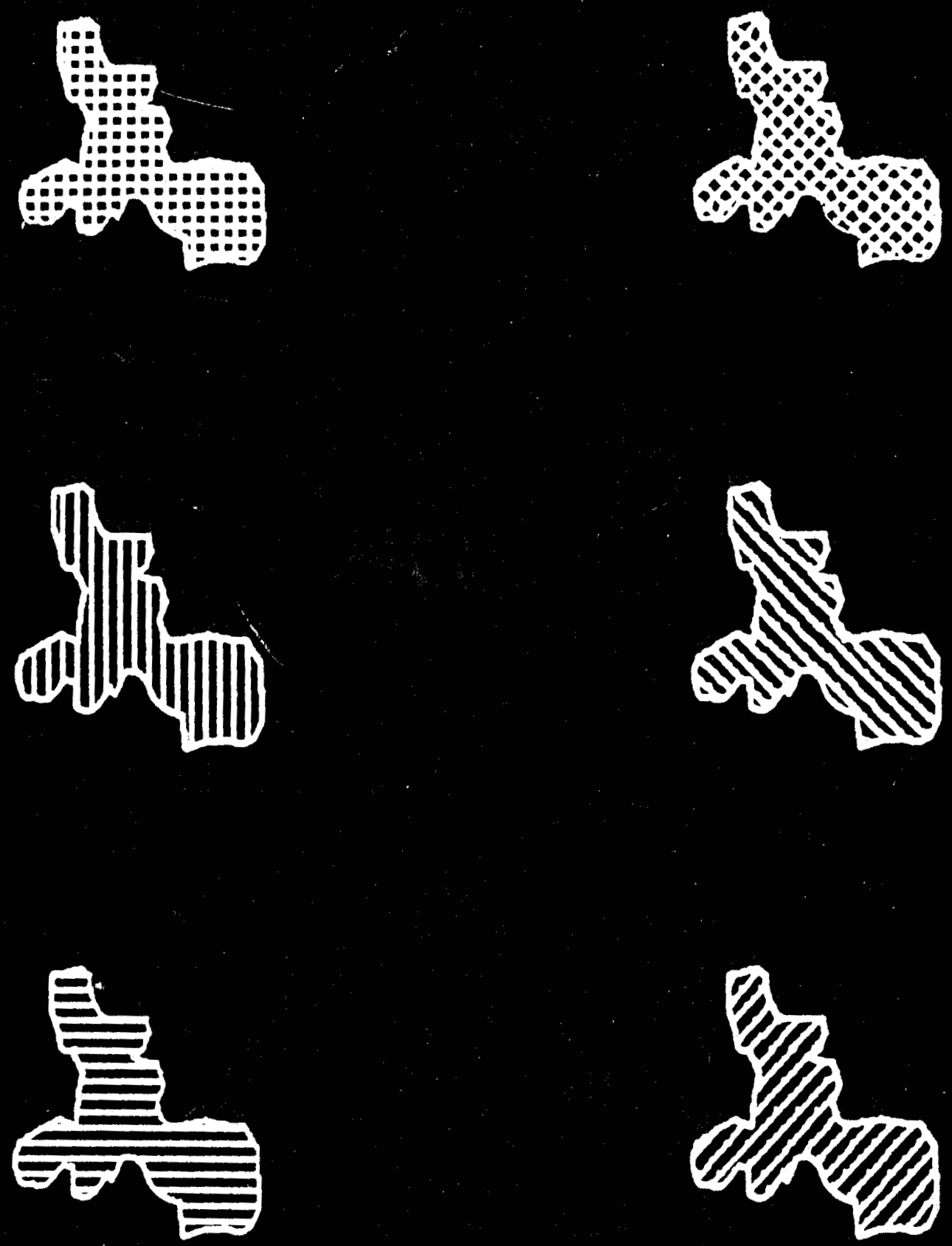

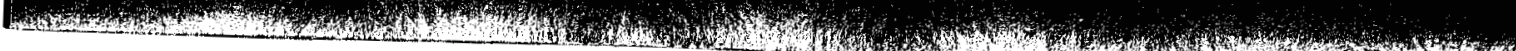

EIGURE 4.6 SIX FILL-IN PATTERN CODES 


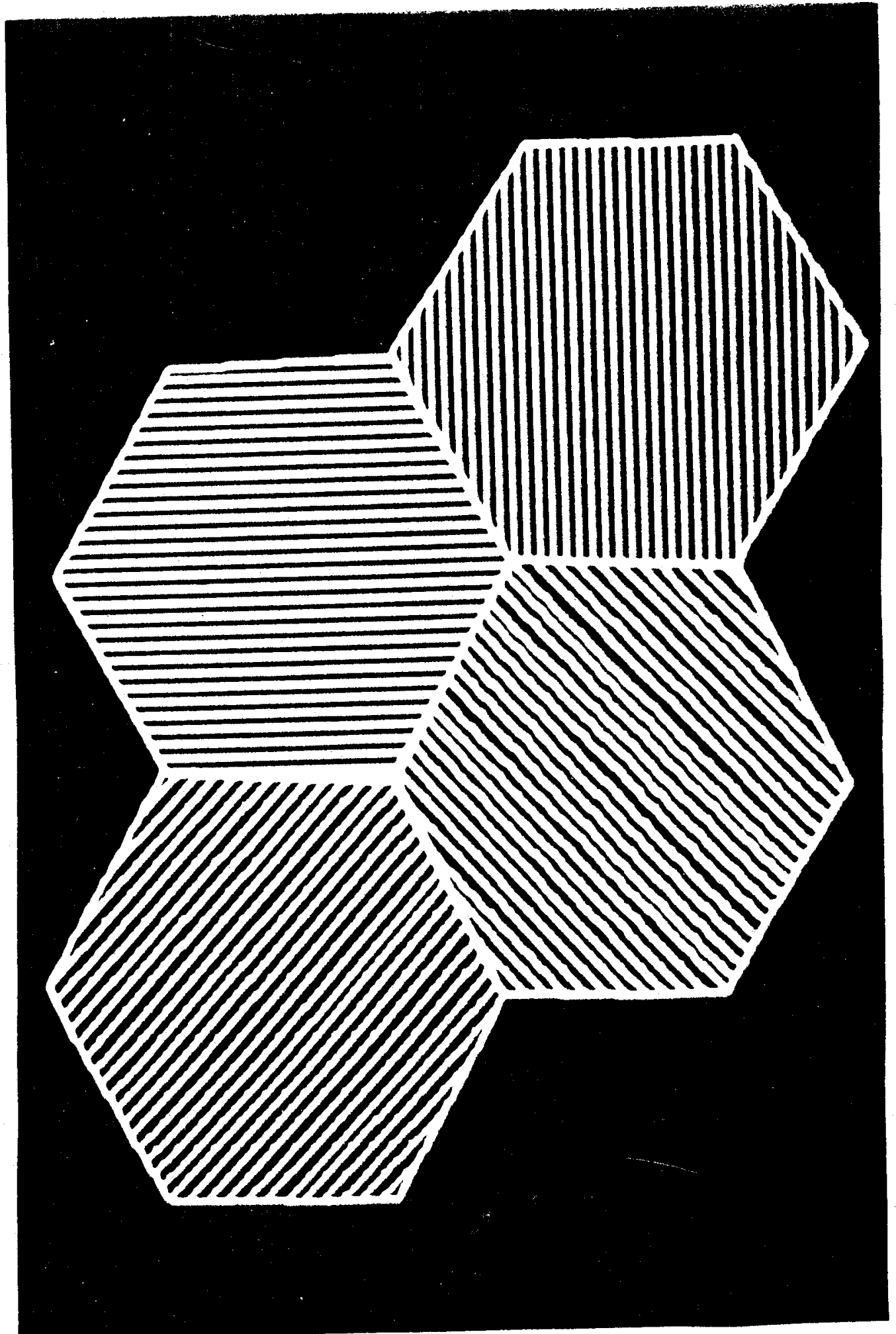

FIGURE 4.7 SAMPIE OF AREA SEPARATION 
optical axis. This produces distortions. Furthermore, the deviation in position between the lens plane and focal plane also affects the quality of the boundary.

In the experimental test, the apparent missing points on some of the film output in area-fill were caused by burns on the CRT phosphor. These burns were made during the early testing of the system. Because of the limitation in the precision of the position control for an image on the CRT light-head, no attempt was made to fill in a large area on a film. 


\section{CHARACTER PRINTING}

\subsection{General}

A photo-typesetter eliminates the time-consuming task of individually tracing each symbol or character. It affords a significant reduction in drafting time because, on the average, thousands of symbols appear on a map. Using a CRT light-head, an almost unlimited magazine of characters is available as they are stored in digital form in the computer memory. The character printing is obtained by moving the light-head to the specified position and imaging the complete filled line character onto the film through the optical lens.

Characters are surrounded by their boundaries. For the application of character printing, the procedures of the 'area-fill' subroutine as described in Chapter 4 can be applied. The boundary data of a character can be obtained directly by digitizing the coordinates of its vertices and storing them into the library file of the computer. Rotation and scaling of a character are obtained by calculating the new coordinate data of the vertices according to the angle of rotation and the magnification vector.

\subsection{Programming}

The simple transformations of translation, rotation, and scaling in the Cartesian graphical system are listed 
as follows (17):

Translation:

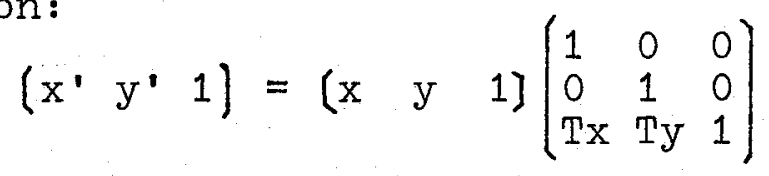

Rotation:

$$
\left[\begin{array}{lll}
x^{\prime} & y^{\prime} & 1
\end{array}\right)=\left(\begin{array}{lll}
x & y & 1
\end{array}\right)\left(\begin{array}{ccc}
\cos \theta & \sin \theta & 0 \\
-\sin \theta & \cos \theta & 0 \\
0 & 0 & 1
\end{array}\right)
$$

Scaling:

$$
\left(\begin{array}{lll}
x^{\prime} & y^{\prime} & 1
\end{array}\right)=\left(\begin{array}{lll}
x & y & 1
\end{array}\right)\left(\begin{array}{lll}
S x & 0 & 0 \\
0 & \text { Sy } & 0 \\
0 & 0 & 1
\end{array}\right)
$$

Where $\left(x^{\prime}, y^{0}\right)$ is the coordinate of a point after transformation

$$
\begin{array}{ll}
(x, y) & \begin{array}{l}
\text { is the coordinate of a point before } \\
\text { transformation } \\
\text { is the distance to be translated in } \\
\text { the X direction }
\end{array} \\
\text { Ty } & \begin{array}{l}
\text { is the distance to be translated in } \\
\text { the } Y \text { direction }
\end{array} \\
\theta & \begin{array}{l}
\text { is the counterclockwise angle of } \\
\text { rotation about the origin of the } \\
\text { system }
\end{array}
\end{array}
$$

Sx \& Sy are the magnification factors in the $X$ and $Y$ direction respectively. They have the effect of distorting characters by elongating or shrinking them along the directions parallel to the coordinate axis.

For the application of character printing, all the coordinates of the vertices of a character are transformed by the translation equation so that their center point ( $\mathrm{Cx}, \mathrm{Cy}$ ) becomes the origin of the coordinate system. (i.e. $\mathrm{Tx}=-\mathrm{Cx} ; \mathrm{Ty}=-\mathrm{Cy})$. The center point $(\mathrm{Cx}, \mathrm{Cy})$ is calculated 
by determining the minimum and maximum values of the character. Scaling is achieved through the scaling equation with the desired values ( $\mathrm{Sx}$ and $\mathrm{Sy}$ ). If the rotation center (Rx, Ry) coincides with the center point ( $\mathrm{Cx}, \mathrm{Cy})$, the rotation equation can then be applied to rotate the character in three-degree increments. If the rotation center is defined other than as the center point, further translation is required so that ( $R x, R y$ ) becomes the origin. (i.e. $T x=-R x$; Ty $=-$ Ry); the rotation equation can then be applied. Finally the point is translated so that the origin is returned to the center point. (i.e. $T x^{\prime \prime}=R x ; T y^{\prime \prime}=R y$ ).

In order to ensure that all characters are generated at the center of the screen ( $\mathrm{Cx}^{\prime}, \mathrm{Cy}^{\prime}$ ) of the CRT light-head, all the points of the character have to be translated so that ( $\mathrm{Cx}, \mathrm{Cy})$ lies on $\left(\mathrm{Cx}^{\prime}, \mathrm{Cy}^{\prime}\right)$.

The area-fill subroutine is then carried out to fill in the bounded area using the light-head for the character printout. The routine of the program is illustrated by Flowchart 5.1 .

\subsection{Advances on previous work}

Character printing using the method of 'area-fill' can provide versatile filling patterns as described in Chapter 4. By calculating each vertex of a character, scaling can be achieved without any limitation as would 


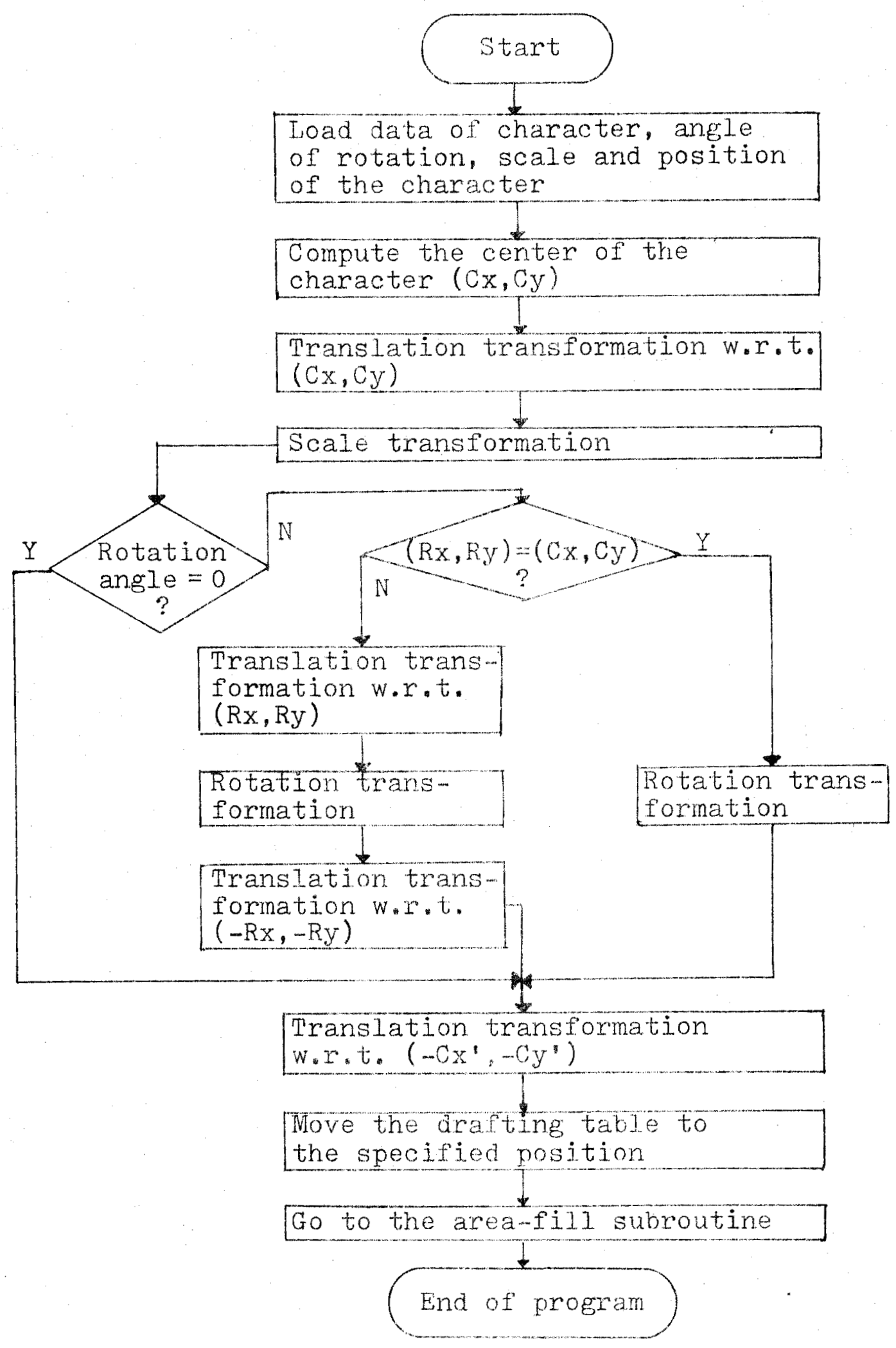

FLOWCHART 5.1 CHARACTER PRINTING PROGRAIM 
occur with Wilkie's(1) method which stores the absolute start and end coordinates of the scan lines forming a character. The area-fill technique provides better results in terms of quality than Wilkie's method. This is partly due to the round-off error in the calculation of rotation. In Wilkie's method, these errors affect all points along the perimeter of a character so that uneven edges may appear on the boundary of the character. On the other hand, in this method the round-off errors have effect only at the vertices of a character and not at the points between them. Furthermore, the slope of filling-lines is independent of the angle of rotation so that the density is constant all the time.

Another advantage of the area-fill. method is that it can select any rotation center by a simple translation transformation. It is more suitable to use a mathematjoal. control for this change. The boundary data is usually most easily obtained in cartographic applications directly by digitization of enlarged characters. The major drawback of this method is the slower filling speed of characters as compared to Wilkie's method. The drawing speed depends on the size and the number of vertices of the character. For example, this method, on the average, takes 4 seconds for each character drawn in Figure $5 \mathrm{~F} .1 \mathrm{~b}$, which is 12 times longer than Wilkie's method for that size of printing. 
5.4 Samples of present work

Figure 5.1 to ligure 5.4 are taken from the Tektronix 611 storage display.

For comparison of the quality obtained by the two different methods, Figure 5.3 is generated by the areafill technique with the same angle of rotation of the character as those shown in Figure 5.4 which was generated by Wilkie's method. Also, the film outputs of the CRT lighthead as shown in Figure $5 \mathrm{~F} .1 \mathrm{~b}$ and Figure $5 \mathrm{~F} .1 \mathrm{a}$ were generated by the area-fill method and Wilkie's method respectively to illustrate the actual performance of the system. 


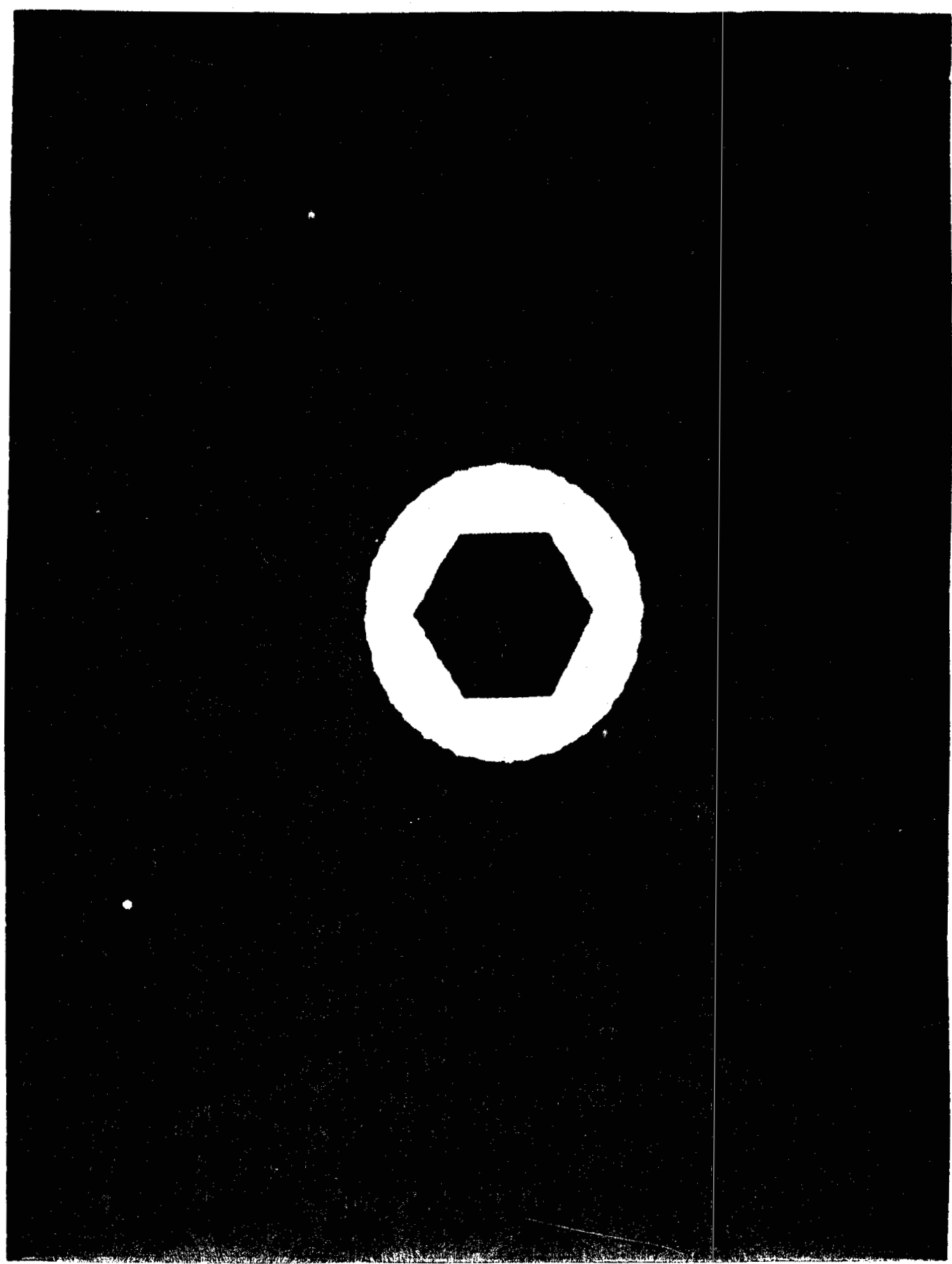

\section{FIGURE 5.1 SAMPLE OF CHARACTER PRINTING (611 display)}




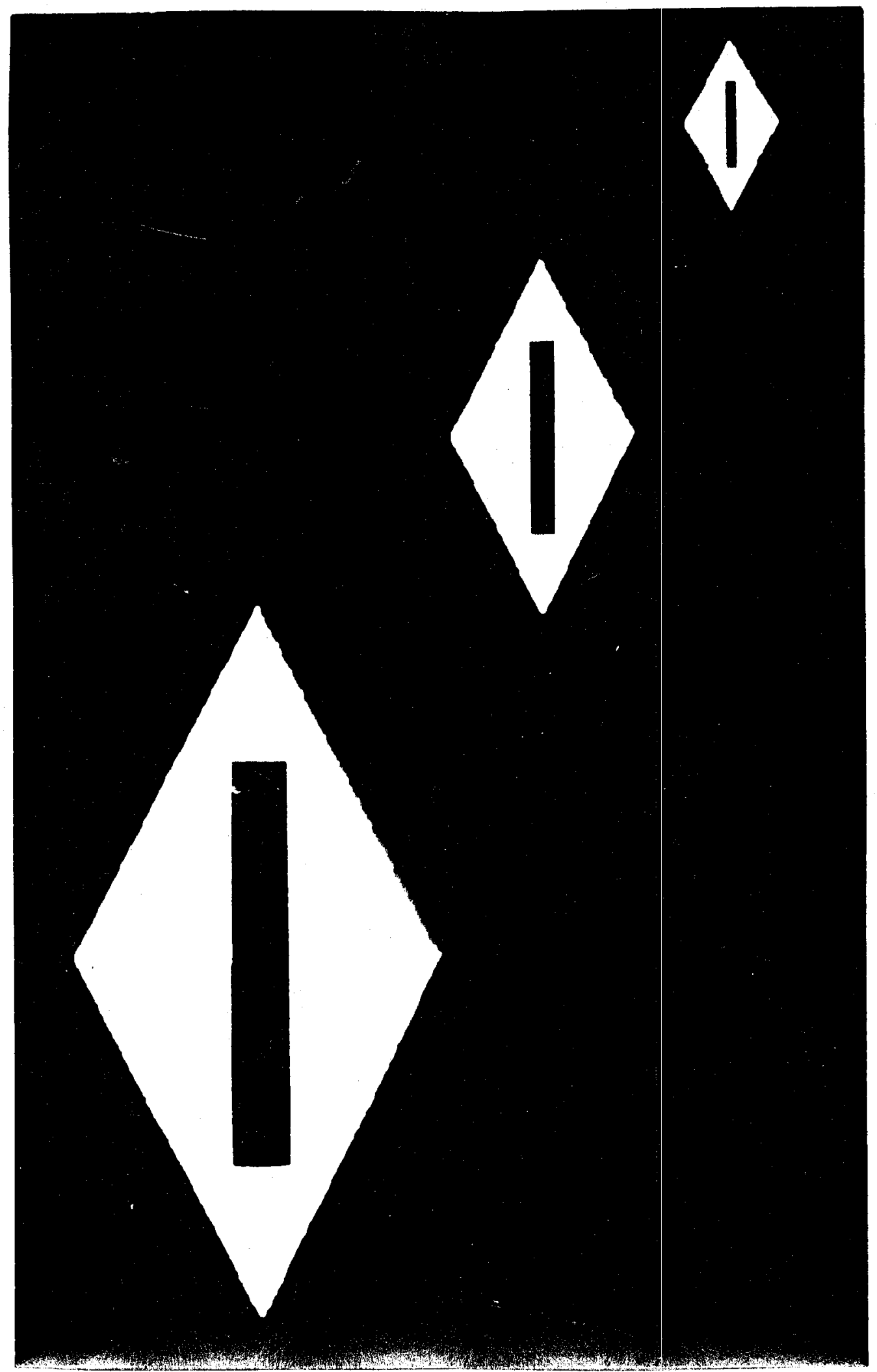

FIGURE 5.2 SAMPLE OF SCALING 


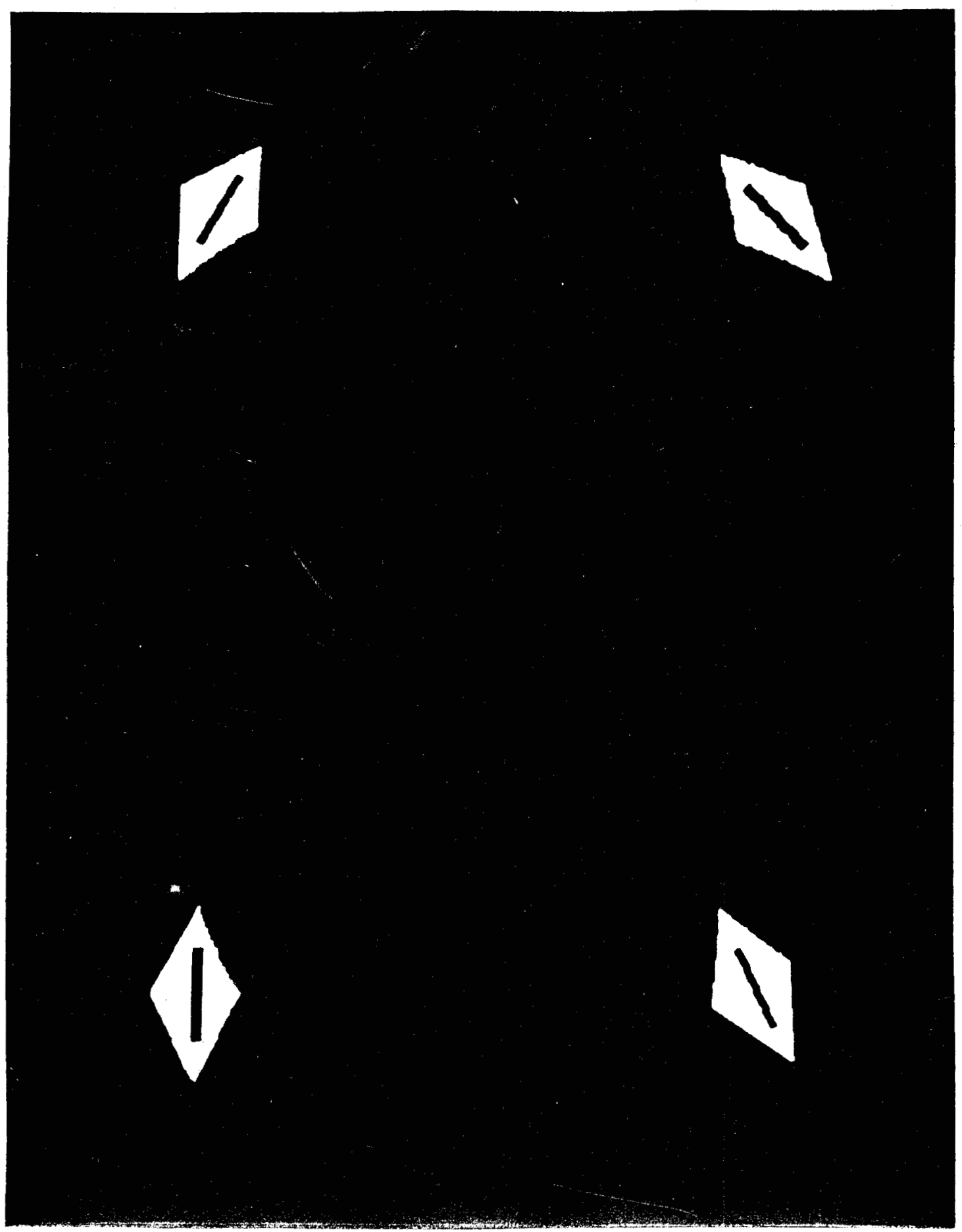

FIGURE 5.3 SAMPLE OF ROTATION (AREA-FILL TECHNIQUE) 


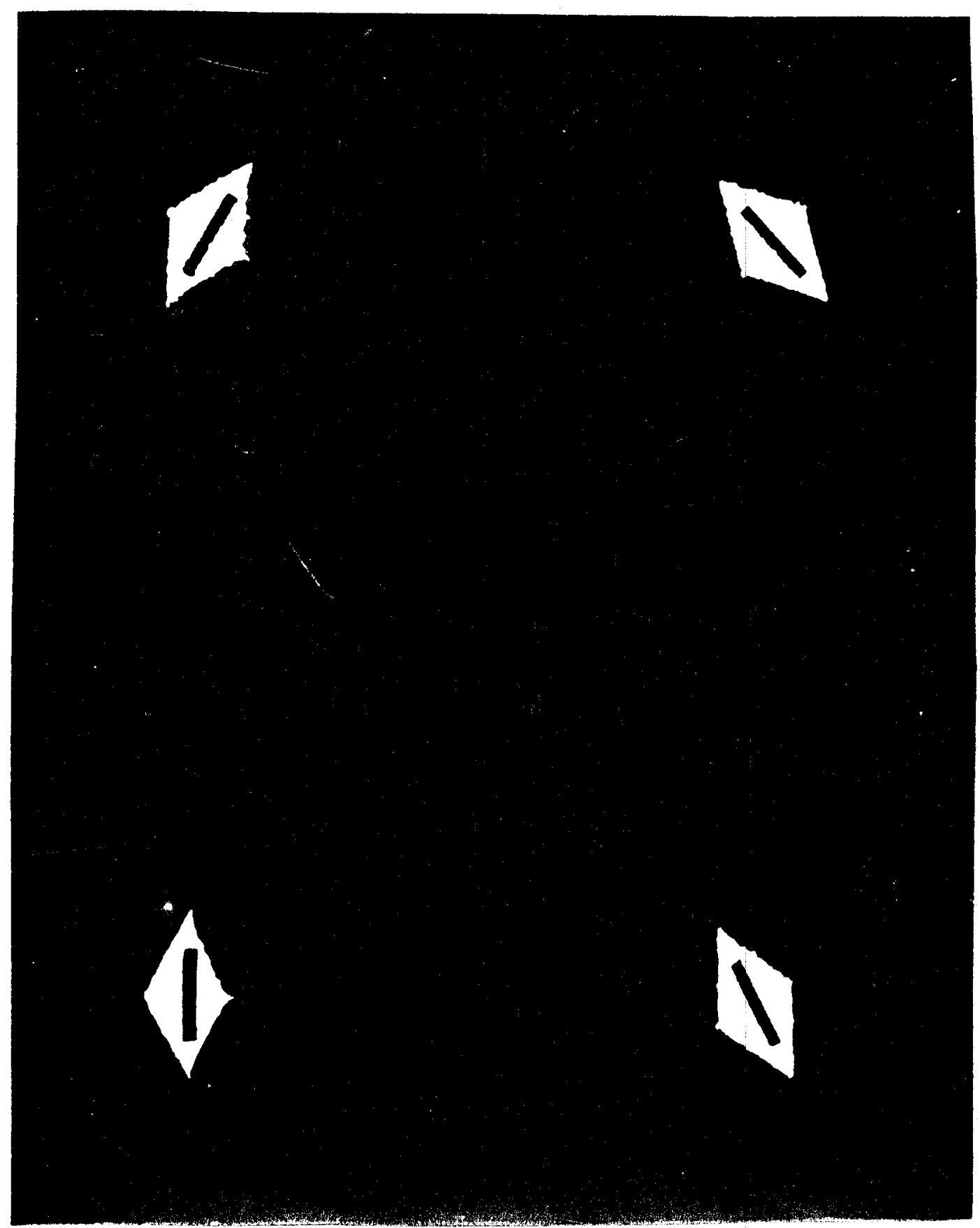

FIGURE 5.4 SAMPLE OF ROTATION (SCAN-LINE FORMAT) 


\section{CONCLUSIONS AND RECOMNENDATIONS}

\subsection{Conclusions}

The objective of this work was to design and implement a CRT light-head for map overlay preparation on an automatic drafting system. Emphasis was placed on the drafting of cartographic lines, symbols, and alphanumeric printing. The system consisted of a Digital Equipment Corporation PDP8/e mini-computer, (with $8 \mathrm{~K}$ words of core memory, an extended arithmetic element for high speed calculation, a magnetic disc storage unit and a 9 track magnetic tape unit), a Gerber Model 22 precision drafting table and its $\mathrm{PDP} 8 / \mathrm{e}$ interface.

Using available equipment (Tektronix Type T5021P1 cathode-ray tube and its associated electronics), the automatic drafting system developed in this thesis has demonstrated the capability of producing drafting of acceptable quality.

A CRT light-head was employed because it removed the limitation of the number of available symbols and increased the precision and speed of rotation as against a conventional opto-mechanical light-head using a light source, a set of mechanical masks and an optical system. Different configurations of light spots for line drafting were considered. The rectangular light spot was selected because it best suited the requirement for square corners 
and it was most easily generated on the screen of the CRT.

A control and interface unit was developed so that no human intervention was required in the drafting process. The dynamic rotation control of the image was produced by software because of its simplicity and accuracy. In addition, the error of rotation by software calculation is non-cumulative.

Various cartographic line widths were achieved by changing the resistance value in the programmable voltage divider of the width control unit.

As the spot size of a CRT is appreciably affected by varying the beam current, the intensity control was obtained by varying the scan repetition rate (frequency) of the image according to the speed of the drafting table. It was found that this kind of control was non-linear, especially at low drafting speed, but was acceptable, mainly because of the large exposure latitude of the photographic materials that were used.

It is not possible to draw the original trace using unmodified digitized data. However, it is possible to define a unique smooth curve for this data. Forward-look Iinear interpolation was applied for this purpose in the system. It was found that this method is suitable not only for data of cartographic and other non-mathematical origins, but also for mathematically derived data for which numerical 
approximations are not usually appropriate.

Several PDP8/e programs were written to confirm that all design goals had been met, and to calibrate the hardware of the system.

Area-fill technique utilizes the familiar topological property that a line crossing a closed boundary contour will intersect the contour an even number of times. Any irregularly shaped image can be determined by its boundary. This scheme was then extended to the character printing as compared to the previous method presented by Wilkie ${ }^{(5)}$. It was found that the area-fill technique is more suitable for the modification of an image by rotation and scaling at the expense of filling speed.

In addition to the block filling pattern, six different filling patterns were developed for the purpose of area symbolization.

\subsection{Recommendations}

The selection of system components has been discussed in section 2.3. Better components would improve the quality of the drafting system. It was also found that the focussing problem due to the distance deviation of the CRT/lens system from the film plane made height adjustments critical. For further applications, it is suggested that the CRT/Iens system be supported by an air bearing riding 
on the table platen. This ensures that the final lens in the light-head's optical system remains at a fixed distance from the surface of the film.

The maximum drafting speed of the system is governed by the intensity of the CRT, the speed of the film, the aperture of the optical lens and the cutoff frequency of the intensity control unit. Therefore, in order to increase the drafting speed, all the above factors have to be considered. Since the characteristics of a CRT vary from unit to unit, further investigation is worthwhile in order to determine an optimum beam spot size and type of phosphor for the drafting system.

Using a precision CRT light-head, the dimension of an image focussed down onto a film can be easily calibrated in terms of the step size of a drafting table so as to handle the 'area-fill' technique for a large area as discussed in Chapter 4.

Programmable voltage levels obtained from digital to analog converters can take the place of the voltage divider of the width control unit. In this way, the end points of a projected image can be determined directly by the input data sent to the digital to analog converters. Various cartographic line patterns (e.g. twin line drafting for roads) could be made available with a CRT light-head by increasing the number of digital to analog converters 
so that more than two voltage levels can be used to specify the end points of an image.

For the convenience of system control, it is suggested that the selection of the operation modes in the line drafting and character printing is by software instead of manual switching as in the proto-type unit. 


\section{REFERENCES}

1. Auerbach Publishers Inc; "Auerbach on digital plotters and image digitizers," 1972.

2. Evangelatos, T.; "Automatic drafting," Canadian Hydrographic Service, January 1970.

3. Curtiss, D.A.; "Automated drafting, where are we now?" Graphic Science, July 1965.

4. Carrillo, P.; "Drafting with Iight," M.Sc. Thesis, University of Saskatchewan, 1974.

5. Wilkie, W.T.; "Computerized cartographic name processing," M.Sc. Thesis, University of Saskatchewan, 1973.

6. Boyle, A.R.; "The quantised line," The Cartographic Journal, December 1970.

7. Shulman, A.R.; "Optical data processing," John Wiley \& Sons, Inc., 1970.

8. Neblette, C.B.; "Photography, its materials and processes," 6 th ed., D. Van Nostrand Company, Inc., Princeton, N.J., 1962 .

9. The Kalvar Corporation; "The Kalvar handbook," Tech. Bull. 104, New Orleans, La.

10. Poole, H.H.; "Fundamentals of display systems," Spartan Book, 1966.

11. Data sheet for CELCO DS5-50-0

12. Data sheet for BETA PD900

13. Tektronix Inc.; "Type 502 oscilloscope instruction manual," Beaverton.

14. Luxenberg, H.R.; Kuehn, R.L.; "Display systems engineering," McGraw-hill Book Company, 1968.

15. Data sheet for GAF ECONOLINE P4 film.

16. Thomas, J.M.; "The four colour theorem," Phil. Penn., 1972.

17. Newman, W.M.; Sproull, R.F.; "Principles of interactive computer graphics," McGraw-hill Company, 1973. 
18. Davis, S.; "Computer data displays," Prentice-Hill, Inc., Englewood Cliffs, N.J., 1969.

19. Tektronix Inc.; "Tektronix products handbook," 1975.

20. Optical Electronics; "Operating and applications manual of model 3337 frequency to voltage converter," U.S.A.

21. Teledyne Philibrick Nexus; "Data sheet for model 4352 average-rms-vector module," U.S.A.

22. Burr-Brown; "Operating amplifiers," McGraw-Hill, 1971.

23. Digital Equipment Corporation; "Small computer handbook," Maynard, Mass., U.S.A. 1973.

24. Digital Equipment Corporation; "Logic handbook," Maynard, Mass., U.S.A., 1972.

25. The Gerber Scientific Instrument Company; "Gerber model 22 table maintenance manual."

26. Sherr, S.; "Fundamentals of display system design," Wiley-Interscience, 1970.

27. Freeman, $H_{\text {.; }}$ "Computer processing of line-drawing images," Computing Surveys, Vol. 6, No. 1, March 1974.

28. McLain, D.H.; "Drawing contours from arbitrary data points," The Computer Journal, Vol. 17, No. 4, November 1974.

29. Gold, M.H.; Turek, J.E.; "Graphic arts symbol generating Hardware for a Gerber Plotting System," CBS Inc., July 1974.

30. Ahlberg, J.H.; Nilson, E.N.; Walsh, J.L.; "The theory of splines and their applications," Academic Press, 1967.

31. Lee, K.P.; "A character generator for cartographic display," M.Sc. Thesis, University of Saskatchewan, 1968. 
APPENDIX A

\section{AUTOMATIC DRAFTING}

\section{A.1 General}

A number of drafting techniques are presently being used in automatic drafting systems. These can be divided into three basic types. The first technique uses an ink pen to draw lines directly onto paper. The second uses a scriber to remove a thin plastic emulsion coating from a transparent mylar base. The third uses the focussed image of a light beam to produce lines directly onto photosensitive material.

Ink pen and scribing techniques are of little use in automatic systems for the following reasons:

a) Disadvantages of ink pen

1. Unreliable due to irregular ink flow.

2. Difficult to control width of lines.

3. Requires constant operator attention.

b) Disadvantages of scriber

1. Tool must be kept clean, and a method (e.g. suction) must be used to remove the plastic swarf left from the scriber.

2. 'Shovel effect' tends to make lines longer than intended.

3. There must be constant operator intervention to check the tool as the pressure of 
the cutting tool on the plastic emulsion is critial.

4. An automatic turret head scriber becomes bulky and complicated if a large number of line widths are required.

Although the problems associated with these methods can be minimized, these devices are still, by far, less satisfactory than a light-head in flexibility of operation and quality of the finished product. Furthermore, by using a light-head, symbols can be produced by the 'flashing' technique which can significantly reduce the drafting time. The other types would have to scribe or draw each character symbol individually. Hence, the light-head was selected over the other two. It is the most versatile and the only one to offer a possibility of full automation. The major disadvantages of using a light-head are:
a) A special darkroom environment has to be con- structed for the drafting table.
b) It requires film processing. Therefore, it cannot produce maps directly.
c) Exposure conditions must be carefully controlled to ensure that high quality results are obtained.

\section{A.2 Drafting with light}

As mentioned above, high quality lines can be 
produced by drafting directly onto the photo-sensitive surface of a film sheet with a focussed image of light. Lines are drafted by moving this image of light across the film and symbols are generated by shaping the beam into the desired configuration and flashing it onto the film. Only the best drafting tables can be used with this method, as the least irregularity in motion becomes immediately apparent to the eye because of the almost perfect regularity of the fine line itself. The parameters of the light-head are as follows:

\section{a) Configuration of light beam}

When using a light-head to produce a line on a film, both the exposure condition and the quality of the line depend upon the optical shape of the projected light beam ${ }^{(4)}$. Round, annular and rectangle light spots are commonly used in automatic drafting systems.

1. Round light spot

A. Advantage:

A round light spot is symmetrical about its central axis and therefore need not be rotated when drawing curved lines.

B. Disadvantages:

1) It is difficult to draw squared corners and line ends. This is 
particularly noticeable when drawing a dashed or 'pecked' line.

2) Exposure conditions vary across the width of a line. Fig. A1 and Fig. A2 show the relative exposure across a straight line and a curved line respectively.

3) The width of line produced is affected when the intensity of light is changed. This is because the threshold of the film being used is reached by more of the exposure line profile for more intense light.

4) The absolute value of exposure time for the circle increases proportionally to its diameter. It is therefore necessary to compensate for this change by varying the intensity of the circle as a function of diameter.

5) The region of 'fuzziness' is significant at the edges of the line.

2. Rectangular light spot A. Advartages:

1) Exposure conditions are ideal in drawing a straight line because they 


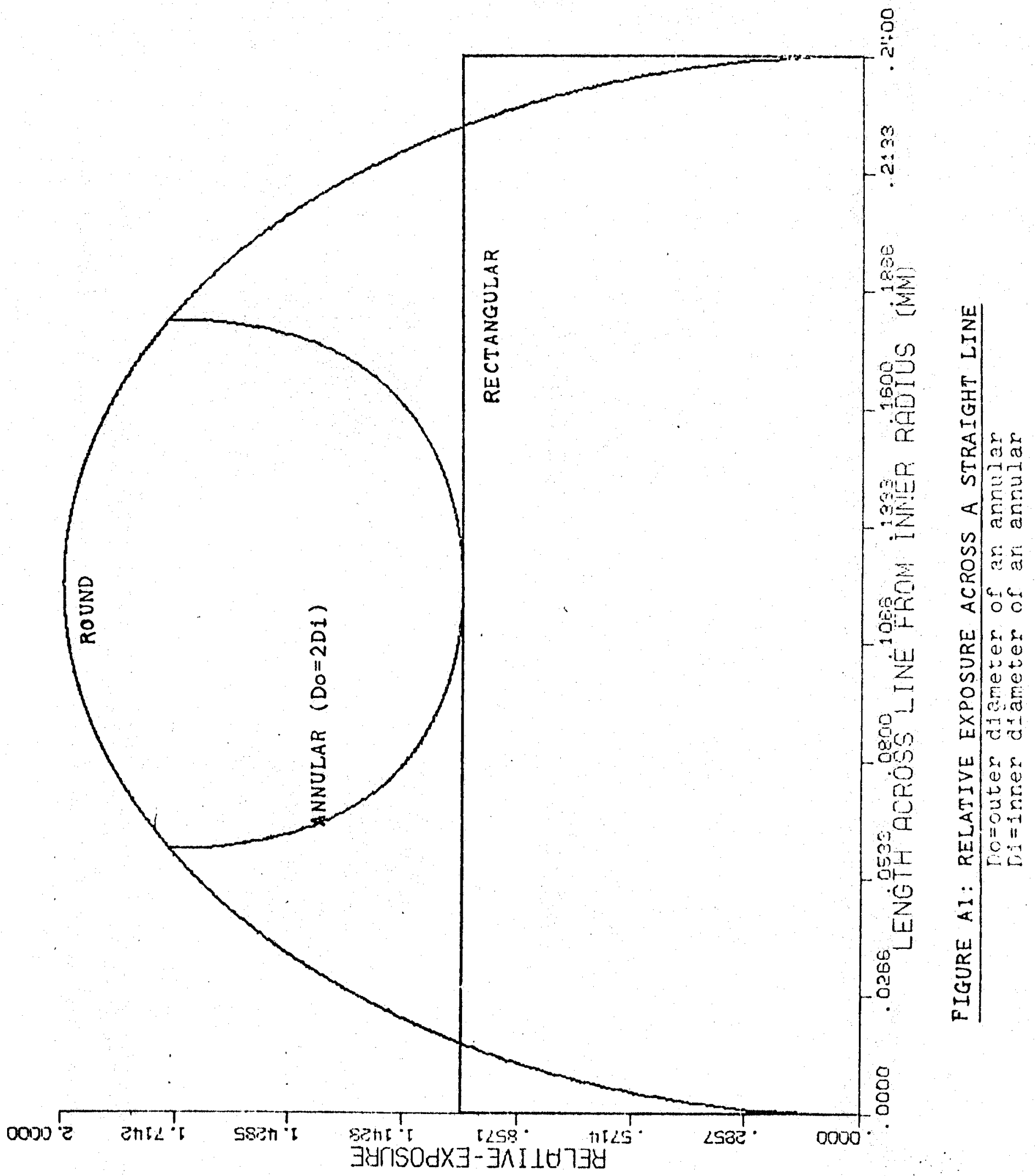




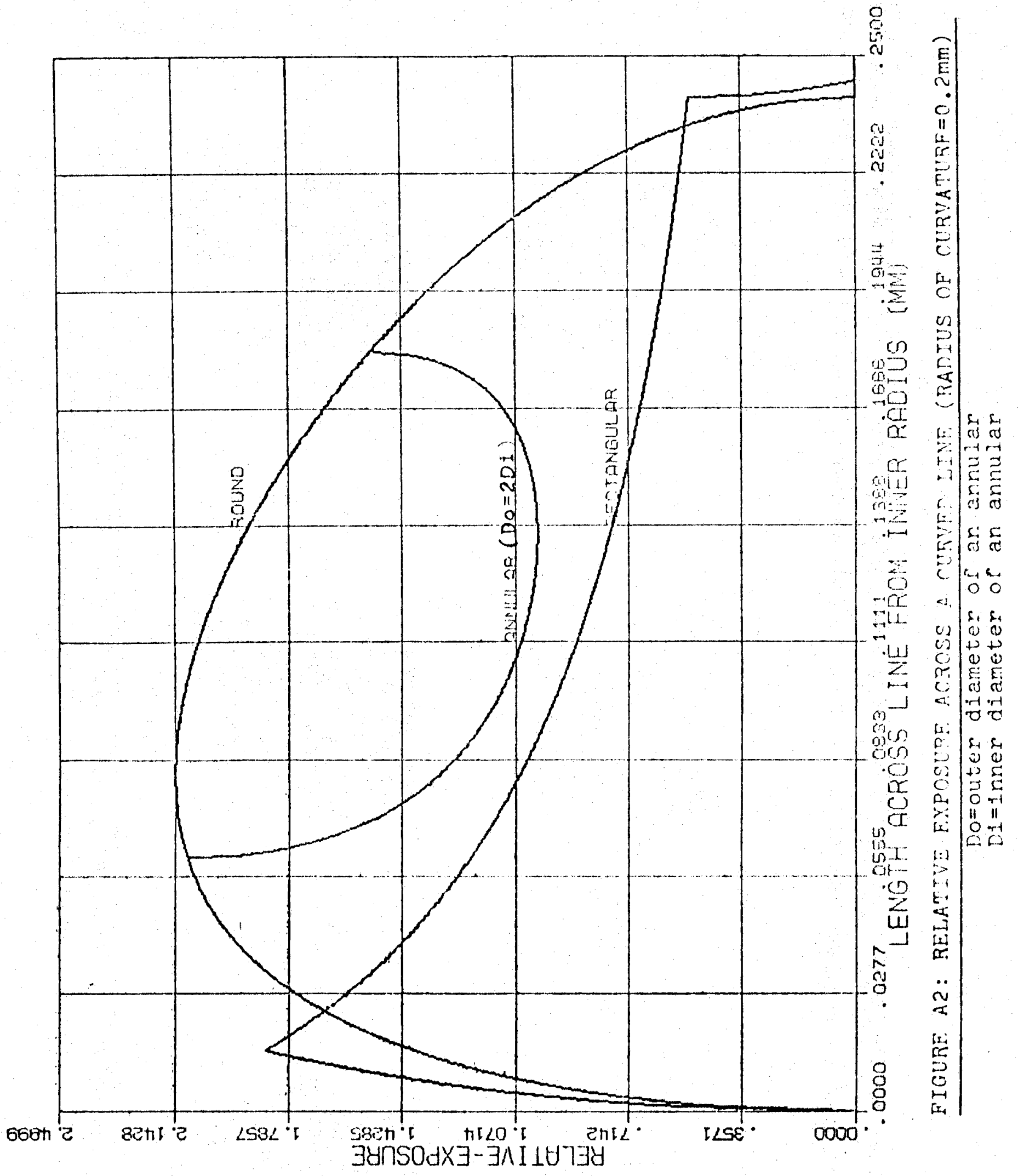


are constant across the width of the line and change abruptly at its edges, therefore the region of 'fuzziness' is negligible.

2) It can produce square corners and line ends.

3) Different line width can be drawn with relative ease because there is no need to compensate for the intensity of the light spot.

B. Disadvantages:

1) Automatic dynamic image rotation must be fitted to maintain constant line width as the line changes direction.

2) The exposure conditions are no longer ideal as a curved line is being traced. (See Fig. A2). Further details about this property can be found in Appendix (C).

3. Annular light spot

A. Advantage:

It has the advantages of a round light spot in that the configuration itself is symmetrical about its central axis. In addition, the exposure condi- 
tions are improved as against a round light-spot. (See Fig. A1 and Fig. A2). This depends on the ratio of outer diameter to inner diameter of the lightspot.

B. Disadvantage:

The major disadvantage of an annular light-spot is that it is difficult to produce squared corners and line ends.

b) Intensity of light-beam

With a certain projected light image for a specified film speed, the maximum speed for drafting is determined by the maximum brightness of the image. Normally a drafting table operates over a range of speeds and the light-head must thus have some type of intensity control in order to produce uniform exposure as the lines are drawn.

c) Drafting process

As mentioned above, light-beams can be used to draw graphical information either by drawing each line individually or by an image flashing technique. Jsually, the image flashing technique is used for character printing but it requires a large capacity magazine in order to have automatic access of many symbols of different 
sizes, scales and orientations. According to the method by which the light-beams are generated, light-heads can be classified into the optomechanical type and the opto-electronic type.

1. Opto-mechanical light-head

Symbols are obtained by shaping rays from the light source into the desired figure through a mechanical mask. The image of this pre-prepared mask is then projected down onto a film by a system of lenses.

The light-head usually uses a tungsten iodide light source or small mercury vapour lamp. A laser beam could also be used as the primary luminous energy source, but no known unit is presently available.

2. Opto-electronic light-head

In an opto-electronic light-head, the symbol image is produced by electronic means when required, and this is imaged onto the photo-sensitive film with a lens. Either a matrix of light-emitting diodes or a precision cathode-ray tube could be used to generate this image. In the former device, each diode would be controlled individually 
so that an image of any shape might be formed. In addition, the main advantage of a light emitting diode matrix is that the elements of symbol image stay at their accurate and stable position. On the other hand, it is difficult to keep uniform illumination of diodes and compensate for device aging.

When a precision CRT is used, the symbol is formed on the screen by carefully controlling the position and intensity of its electron beam. The advantage of a CRT light-head is that there is no limit to the number of shapes that can be produced. A CRT lighthead can provide a good resolution (40 lines per $\mathrm{mm}$ ); however, it is still poor in linearity as compared to an opto-mechanical light-head unless an expensive precision CRT unit is used. 
APPENDIX B

FORWARD-LOOK LINEAR INTERPOLATION

In digital cartography, a Iine is digitized and recorded in the manner of a coordinate of two variables, giving coordinate values at the grid points of a regular rectangular mesh. Specifically we let $(x, y)$ be the coordinate, and we let the coordinate values be the quantities

$$
\left(x_{p}, y_{q}\right)=\left(p d_{x}, q d_{y}\right), 1 \leqslant p \leqslant n_{x} ; 1 \leqslant q \leqslant n_{y} .
$$

Where $d_{x}$ and $d_{y}$ are spacings between grid lines in the $X$ and $Y$ directions respectively, $n_{x}$ and $n_{y}$ are the number of grid lines in the $X$ and $Y$ directions respectively.

The width of cartographic lines generally varies from $0.1 \mathrm{~mm}$ to $0.5 \mathrm{~mm}$ and the absolute accuracy of the position of the center line relative to the map is usually accepted by cartographers as being no better than $0.1 \mathrm{~mm}$ when drawn or traced by hand ${ }^{(6)}$. For this reason, a matrix size $\left(d_{x}\right.$ and $d_{y}$ ) of $0.1 \mathrm{~mm}$ is normally used in digitizers which means that any quantized point is within $0.05 \mathrm{~mm}$ of the original line center. (Fig. B1).

With a rectangular image on the screen of a CRT light-head, a simple way for line regeneration would be to interpolate straight lines between these matrix points (Fig. B2). This is certainly not a true representation of the original line and the irregularities are obvious to the 
viewer: such a line is rough. This is because any curved line is, in fact, composed of short straight segments and small 'gaps' (see (A) in Fig. B2) are produced at the joints of these segments. This effect, due to the rapid changes of 90 degree and 45 degree between line segments, becomes more apparent as the width of a line is increased.

The problem of making a digital computer generate the coordinates of a smooth curve passing through a discrete set of points $P_{k}$ with coordinates $\left(x_{p k}, y_{q k}\right)$ arises frequently in practice. Schemes of varying sophistication and complexity are used; some join the points by straight lines and arcs of circles, others use spline interpolations combined with axis rotation to counteract the inherent limitations of any scheme which expresses $y$ as a polynomial in $x$. Others again use spline interpolation to express the $\mathrm{x}$ and $\mathrm{y}$ coordinates of the curve parametrically.

Certain desirable properties of an algorithm can be specified. It should be invariant under axis rotation, and this can be achieved by treating the $\mathrm{x}$ and $\mathrm{y}$ coordinates symmetrically. The choice is thus between an implicit equation or a parametric equation for the curve. Since an implicit equation may be computationally awkward, the algorithm should give the $\mathrm{x}$ and $\mathrm{y}$ coordinates parametrically. Also, the algorithm should be comprehensive, and if possible, computationally simple. 
It is much more reliable to calculate the data. points required for a smooth effect on a sub-matrix appreciably smaller then $0.1 \mathrm{~mm}$ and then operate the drafting unit under definite position control. The Gerber 22 drafting unit is a purely digital device, and precisely moves $0.0127 \mathrm{~mm}$ ( 0.005 inch) each time a pulse is received from the computer. Using this precise input control with steps or increments so small that they cannot be detected by the eye, interpolation becomes a problem of programming choice. Linear interpolation is usually relatively simple in calculating but, as already mentioned, is not sufficiently good when only used to join specified matrix points on a $0.1 \mathrm{~mm}$ grid.

A 'forward-look' linear interpolation (6) can be then be applied for contour plotting. In this method, each time $1 / \mathrm{N}$ of the distance for a $\mathrm{N}$ point look-ahead is completed, a new aiming point is generated on a logical basis (Fig. B3). The coordinates of the new aiming points of the line created in this way become

$$
\left(x_{p}, y_{q}\right)=\left(\frac{p_{k+N}-p_{k}}{N} d_{x}, \frac{q_{k+N}-q_{k}}{N} d_{y}\right) .
$$

Where $p_{k}$ and $q_{k}$ are the number of grid line of the $(\mathrm{k})$ th point of the line in the $\mathrm{x}$ and $\mathrm{y}$ directions respectively, $p_{k+N}$ and $q_{k+N}$ are the numbers of the grid line of the $(k+N)$ th point of the line in the $\mathrm{x}$ and $\mathrm{y}$ directions respectively. $\mathrm{N}$ is an integer which represents the number of look-ahead points used. 


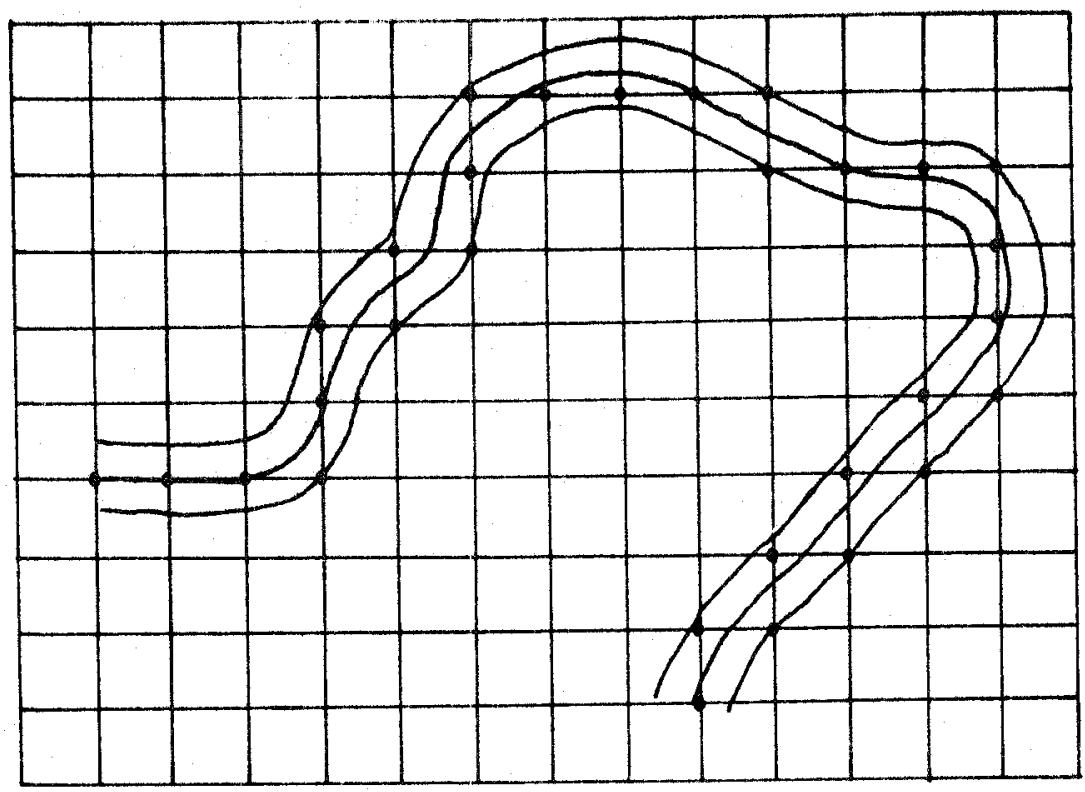

FIGURE B1 QUANTISED COASTLINE ON $0.1 \mathrm{~mm}$ MATR IX

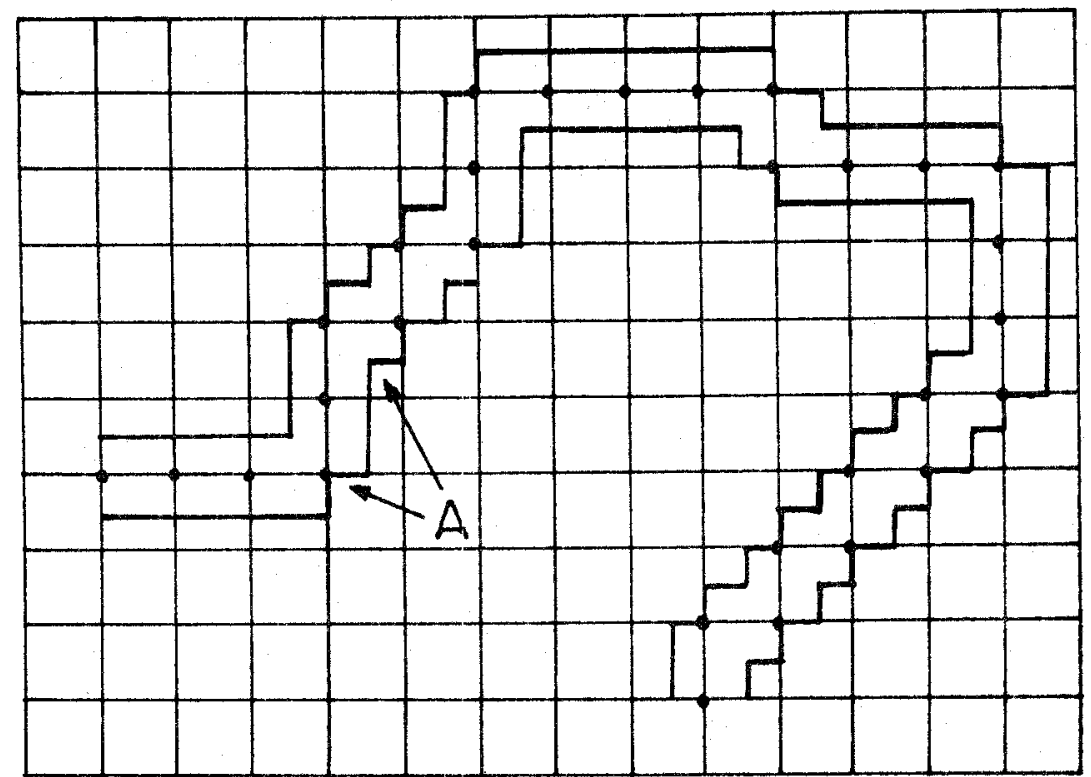

FIGURE B2 LINE REGENERATION BY POINT TO POINT LINEAR INTERPOLATION 


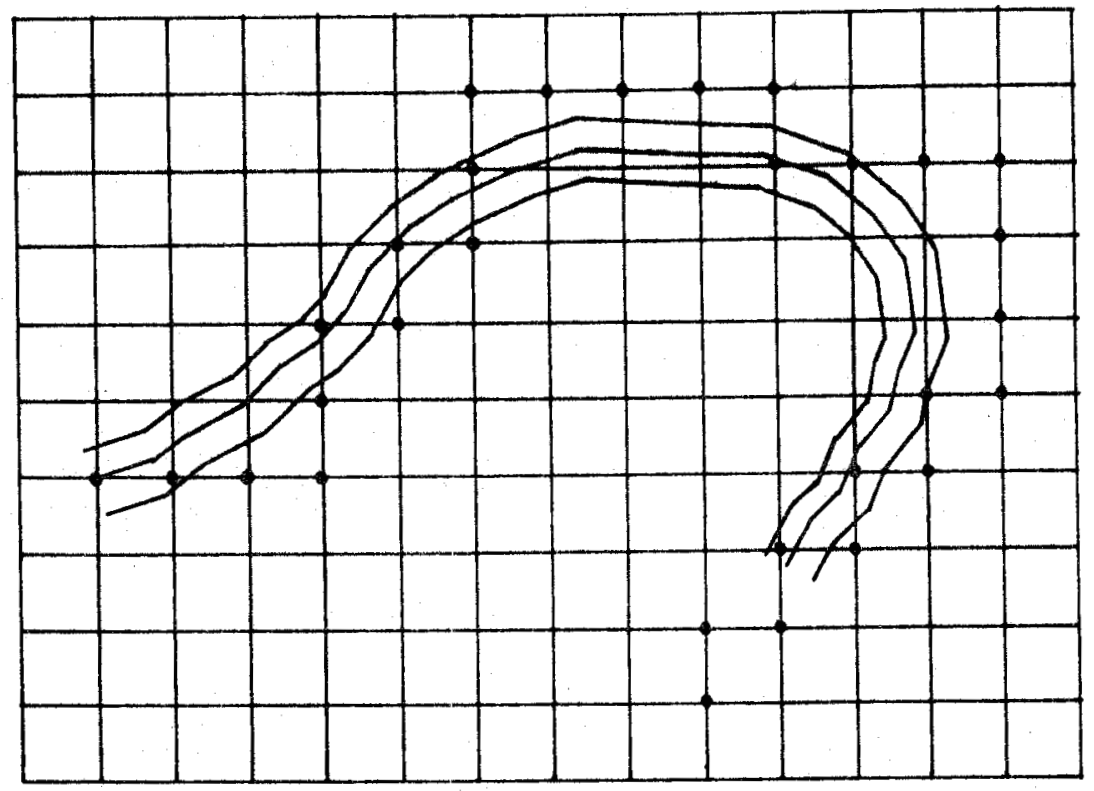

4-POINT

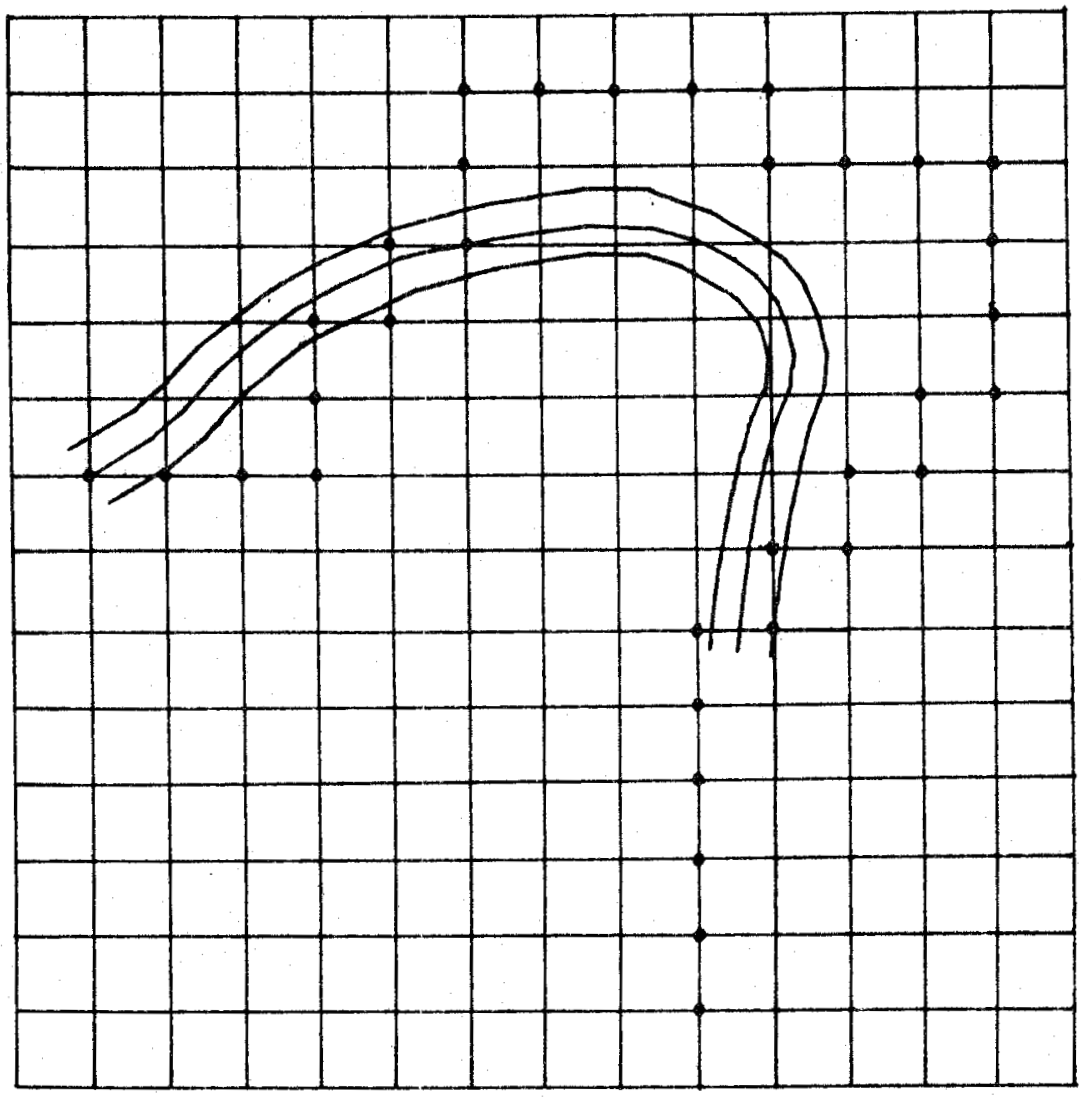

8-POINT

FIGURE B3 4-POINT AND 8-POINT LOOK-AHEAD LINE REGENERATION 
From Figure B3, it can be noted that the lines generated by this look-ahead method are composed of a series of vectors approximately $0.1 \mathrm{~mm}\left(d_{x}, d_{y}\right)$ in length but varying only by a small angle from one to the next; this appears to be most acceptable for visual appearance and is certainly definite and accurate. It is also found that the four-point look-ahead is a reasonable approximation in appearance and accuracy to the original for coastlines. On the other hand, an eight-point look-ahead results in a large error in position and distorts the actual shape of the original. Of course, the selection depends upon the resolution of the digitized data and upon the purpose of the line regeneration. The application of the forward-look linear interpolation in line drafting eliminates the poor appearance of line regeneration due to the rapid changes in direction of the drafting unit. In addition, 'gaps' at the joints of line segments can also be reduced by the smoothness of the line. From Figure BF.1 to Figure $\mathrm{BF} \cdot 3$, maps with and without interpolation can be compared.

This forward-10ok method is intended to enable the user of an incremental drafting table, such as the Gerber 22, to pass a smooth continuous curve through a set of points, instead of merely joining them by a sequence of chords. It supplies to the drafting table the coordinates of a sequence of intermediate points at a chosen regular ratio on a smooth 
curve passing near the data points. It is not possible to set down precise conditions on the deviation of the line from the data points to ensure a visually acceptable and technically valid curve. The algorithm should provide a line which has an acceptable compromise between accuracy and visual appearance. 
APPENDIX C

CHARACTERISTICS OF A RECTANGULAR LIGHT SPOT

The characteristics of a light spot can be determined by the relative exposure across a cartographic line. As derived in Carrillo ${ }^{(4)}$, the relative exposure across a line is a function of the dimensions of the light spot and the radius of curvature of the line being drawn. Varying these parameters, this Appendix describes the relative exposure across a line drafted with a rectangular light spot. It is assumed that the intensity of a projected image is uniform and that the rectangular light spot is being rotated smoothly so that one of its dimensions (the length of a scan-line) is always facing in the direction of tracing.

According to Carrillo(4), the three different regions of a rectangular light spot exposure have different exposure expressions. Each region is defined in terms of the distance from the center of the radius of the curved line being drawn. The expressions which follow have been normalized to the exposure expression of a straight line drawn with a rectangular light spot of width $2 W_{\text {norm }}$ (see Fig. C1).

$$
\begin{array}{ll}
\text { Region 1: } & \left(R_{c}-L\right) \leqslant R \leqslant \sqrt{W^{2}+\left(R_{c}-I^{2}\right)^{2}} \\
& E_{1}=\frac{R_{C}}{W_{\text {norm }}} \cos ^{-1}\left(\frac{R_{c}-L}{R}\right)
\end{array}
$$




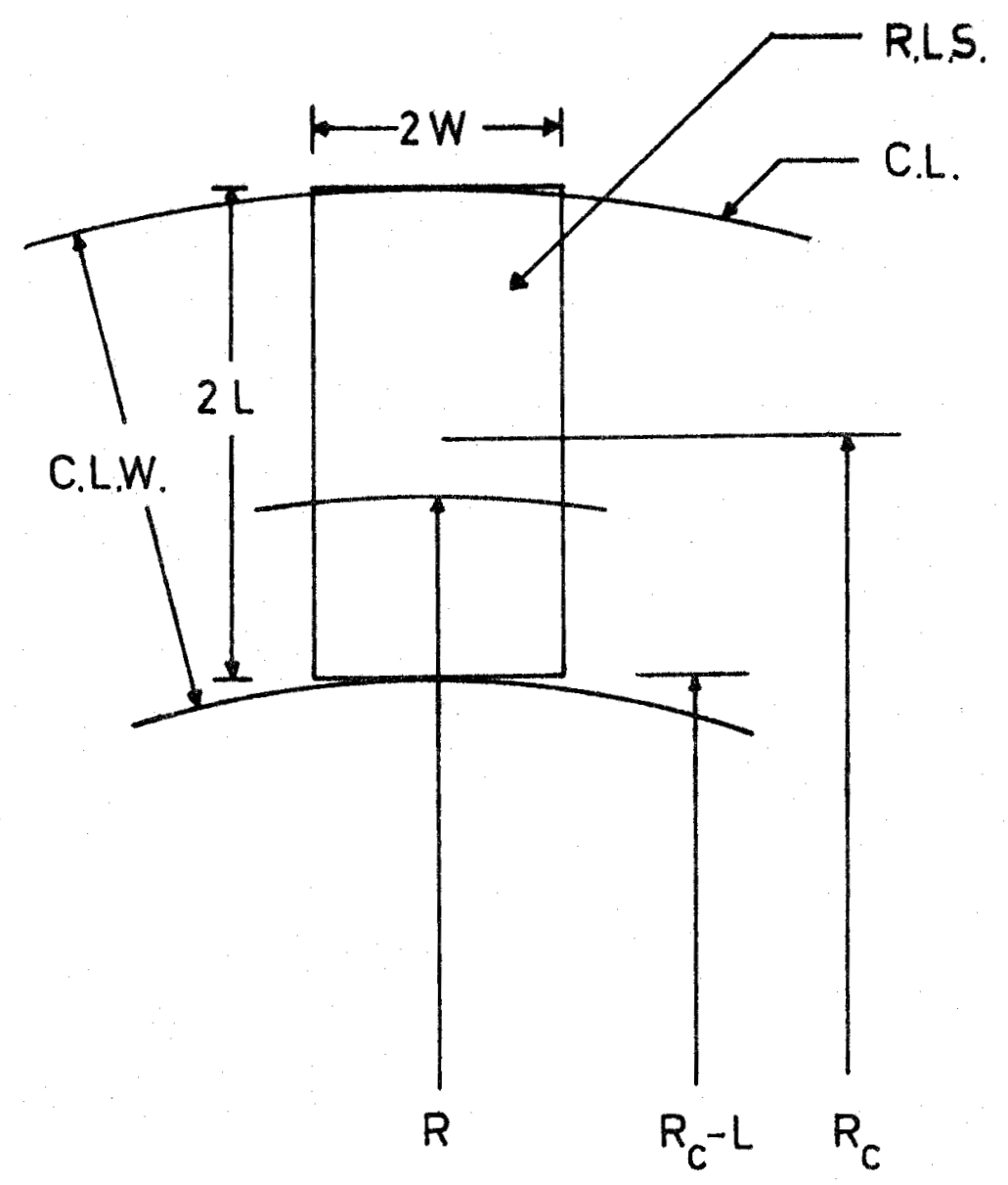

$$
\begin{aligned}
& \text { NOTE: } \\
& \text { C.I.W. = CARTOGRAPHIC } \\
& \text { IINE WIDTH } \\
& \text { R.I.S. = RECTANGULAR } \\
& \text { LIGHT SPOT } \\
& \text { C.I. = CARTOGRAPHIC } \\
& \text { LINE }
\end{aligned}
$$

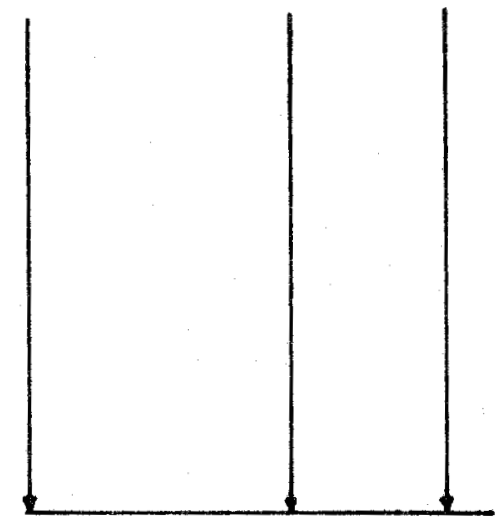


Region $2: \sqrt{W^{2}+\left(R_{c}-L\right)^{2}} \leqslant R \leqslant\left(R_{c}+L\right)$

$$
E_{2}=\frac{R_{c}}{W_{\text {norm }}} \sin ^{-1}\left(\frac{W}{R}\right)
$$

Region 3: $\left(R_{c}+L\right) \leqslant R \leqslant \sqrt{W^{2}+\left(R_{c}+L\right)^{2}}$

$$
E_{3}=\frac{R_{c}}{W_{\text {norm }}}\left(\sin ^{-1}\left(\frac{W}{R}\right)-\cos ^{-1}\left(\frac{R_{c}+L}{R}\right)\right)
$$

Where $2 W=$ width of the rectangular light spot,

$$
\begin{aligned}
2 \mathrm{~L}= & \begin{array}{l}
\text { length of the rectangular light spot; } \\
\text { This sets the cartographic line width, }
\end{array} \\
\mathrm{R}_{\mathrm{c}}= & \text { radius of curvature of the line to } \\
& \text { center of the rectangular light spot, } \\
2 \mathrm{~W}_{\text {norm }}= & \text { width of the normalized rectangular } \\
& \text { light spot. }
\end{aligned}
$$

With $W_{\text {norm }}$ equal to $0.06 \mathrm{~mm}$, the relative exposure of a rectangular light spot is plotted against $W, L$ and $R_{c}$ in Figures $\mathrm{C} 2, \mathrm{C} 3$ and $\mathrm{C} 4$ respectively according to the above expressions.

From Figure C2, it is noticed that the smaller the width of the light spot (W), the more uniform the exposure across a cartographic line and the less deviation of the width of a cartographic line. However, a narrow rectangular light spot produces a low relative exposure. The intensity of the light spot, therefore, must be increased to maintain the drafting speed when using a small value of $W$.

Figure $\mathrm{C} 3$ shows that the relative exposure varies with the radius of curvature of the line being drawn. This property affects the quality of a light-head system in line 


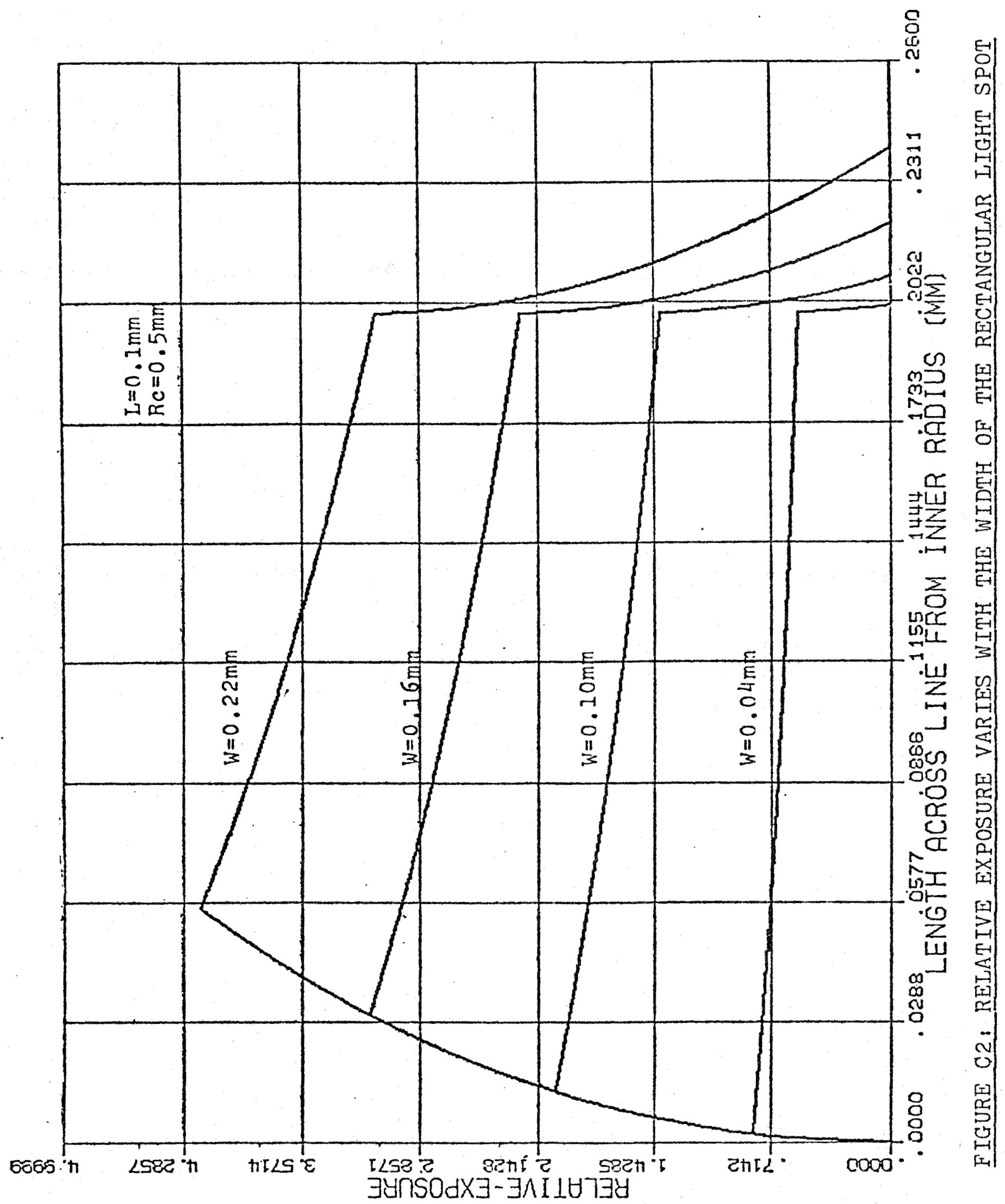




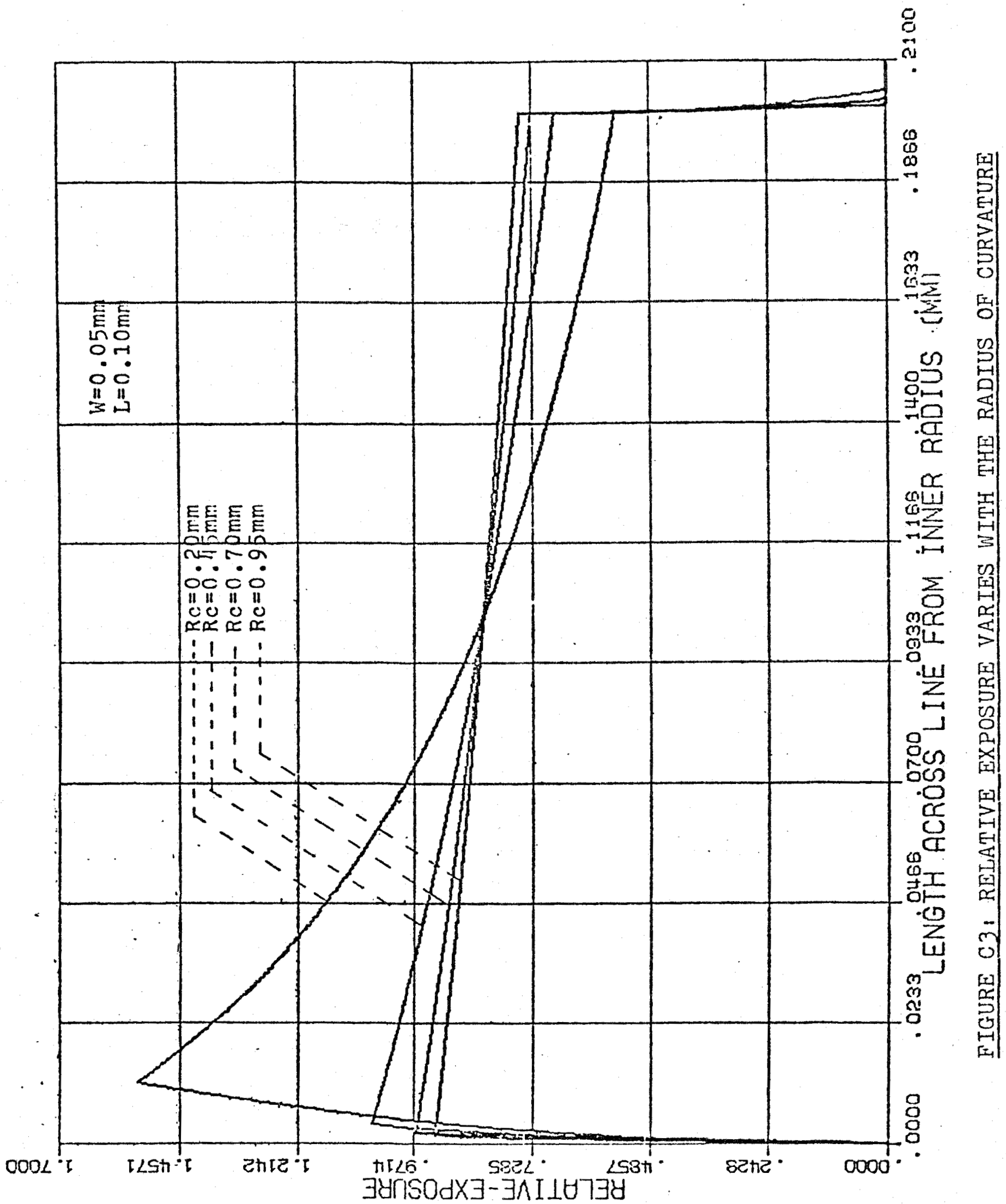




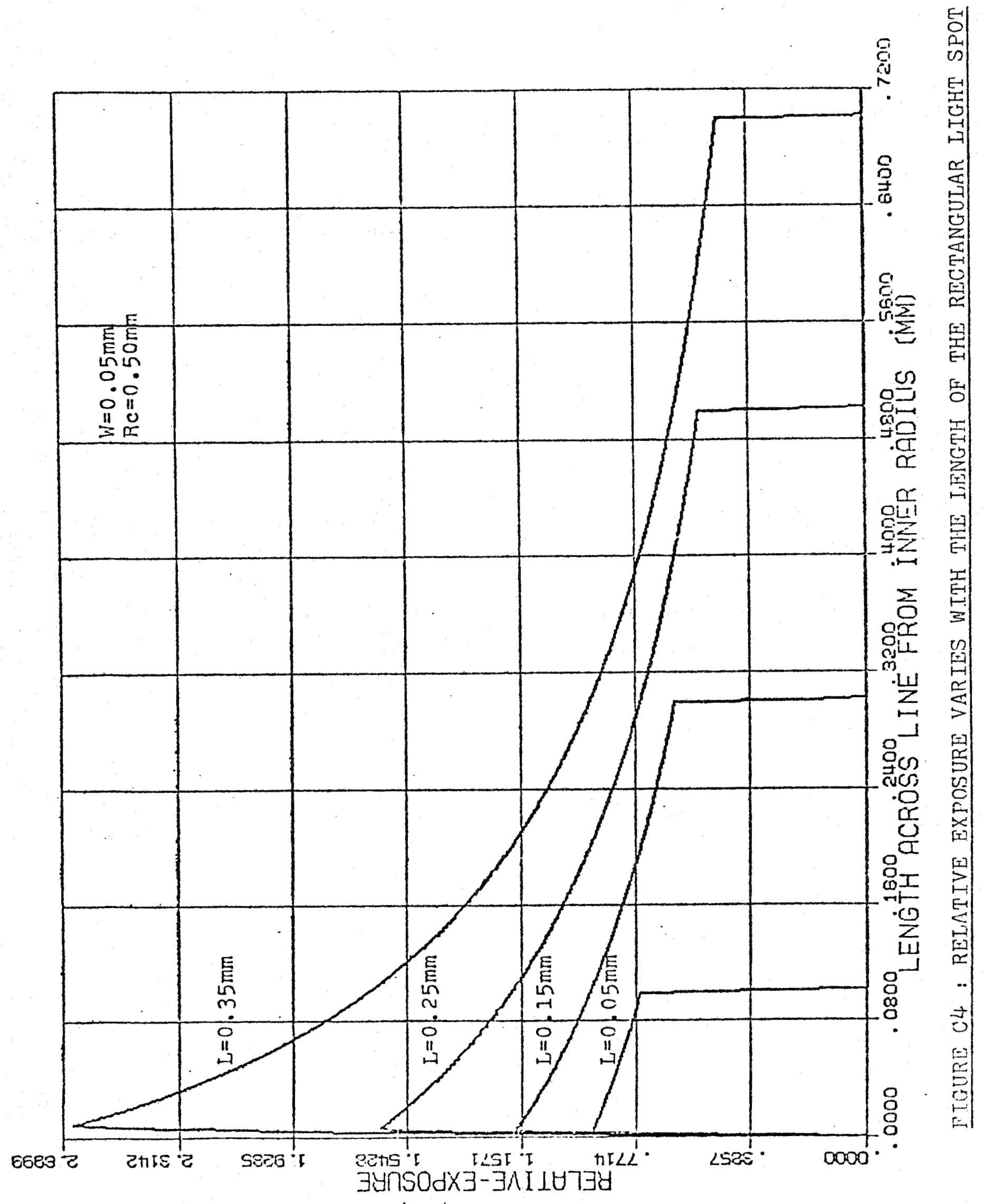


drafting, especially in the drafting of a sharp change in a line. This fluctuation can be reduced by filtering the line data through the forward-look linear interpolation as described in Appendix (B).

Figure $\mathrm{C} 4$ shows a great variation in the exposure across a thick Iine. This is due to the multi-exposures of the projected image and it can only be reduced by using large exposure latitude film. 
APPENDIX D

SCHEMATIC DIAGRAM OF THE PREVIOUS CHARACTER GENERATION UNIT Figures D1 to D5 are adapted from Wilkie ${ }^{(5)}$ to show the schematic diagrams of the character generation unit used both in the earlier and present CRT Iight-head systems. It consists of five circuit boards, namely the device selector board, the control logic board, $\mathrm{X}$ data buffer board, $Y$ data buffer board and the digital to analog converter board. Information about the functions is given in Reference (5). 

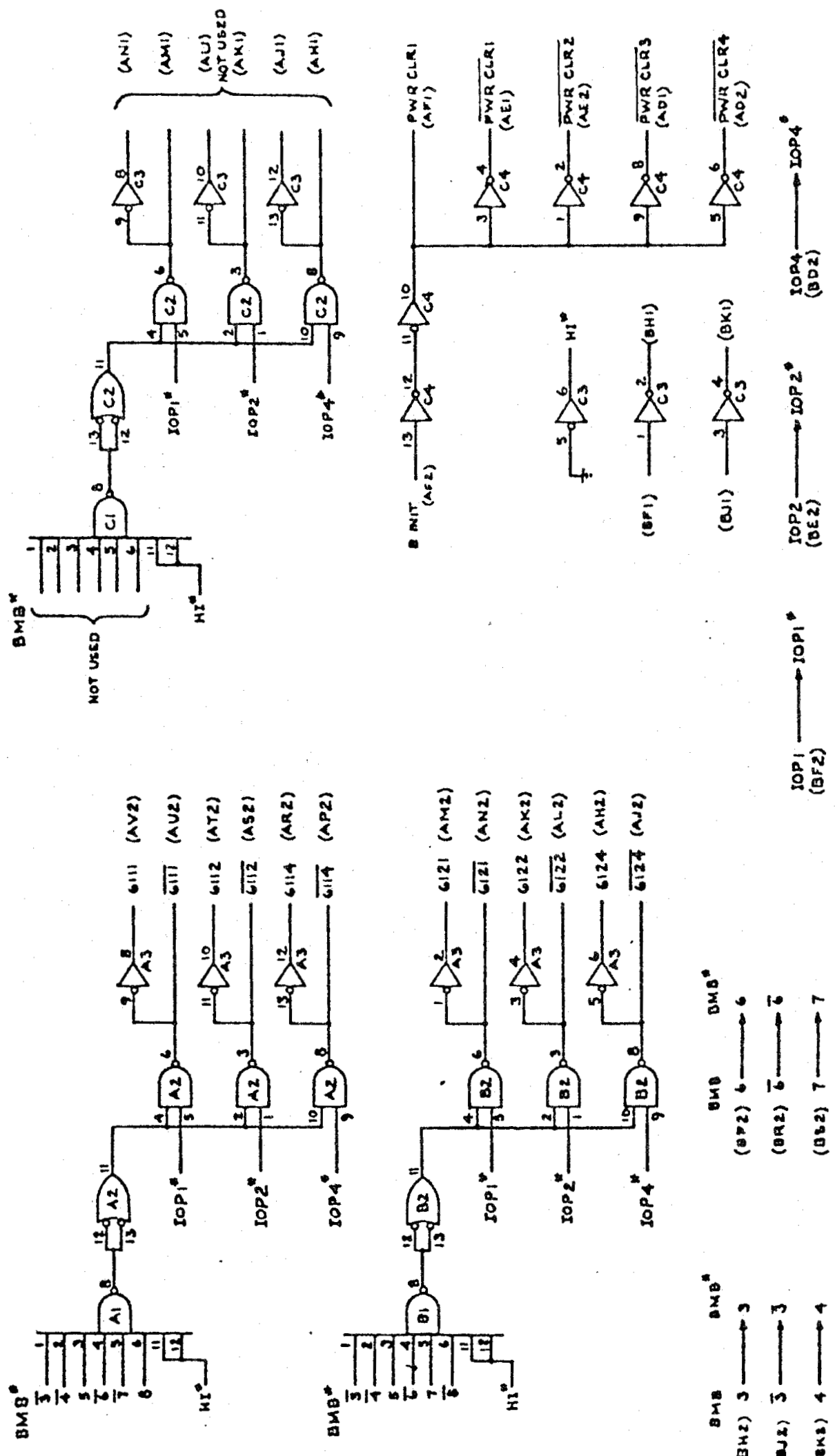

ให้

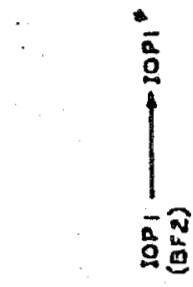

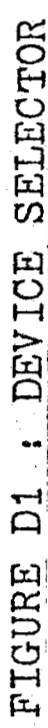

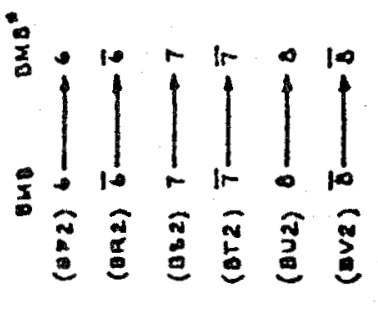

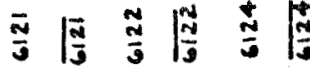
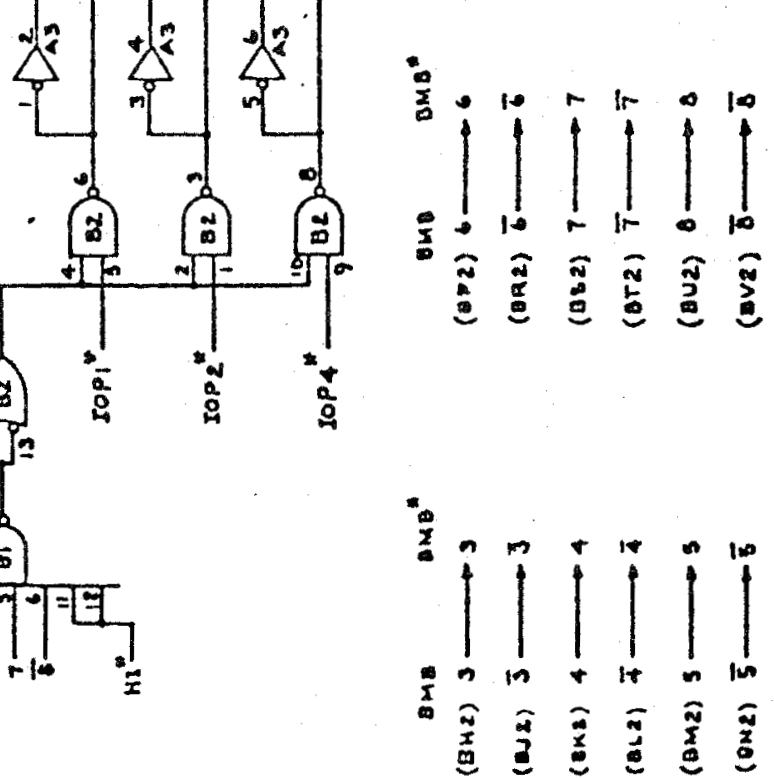


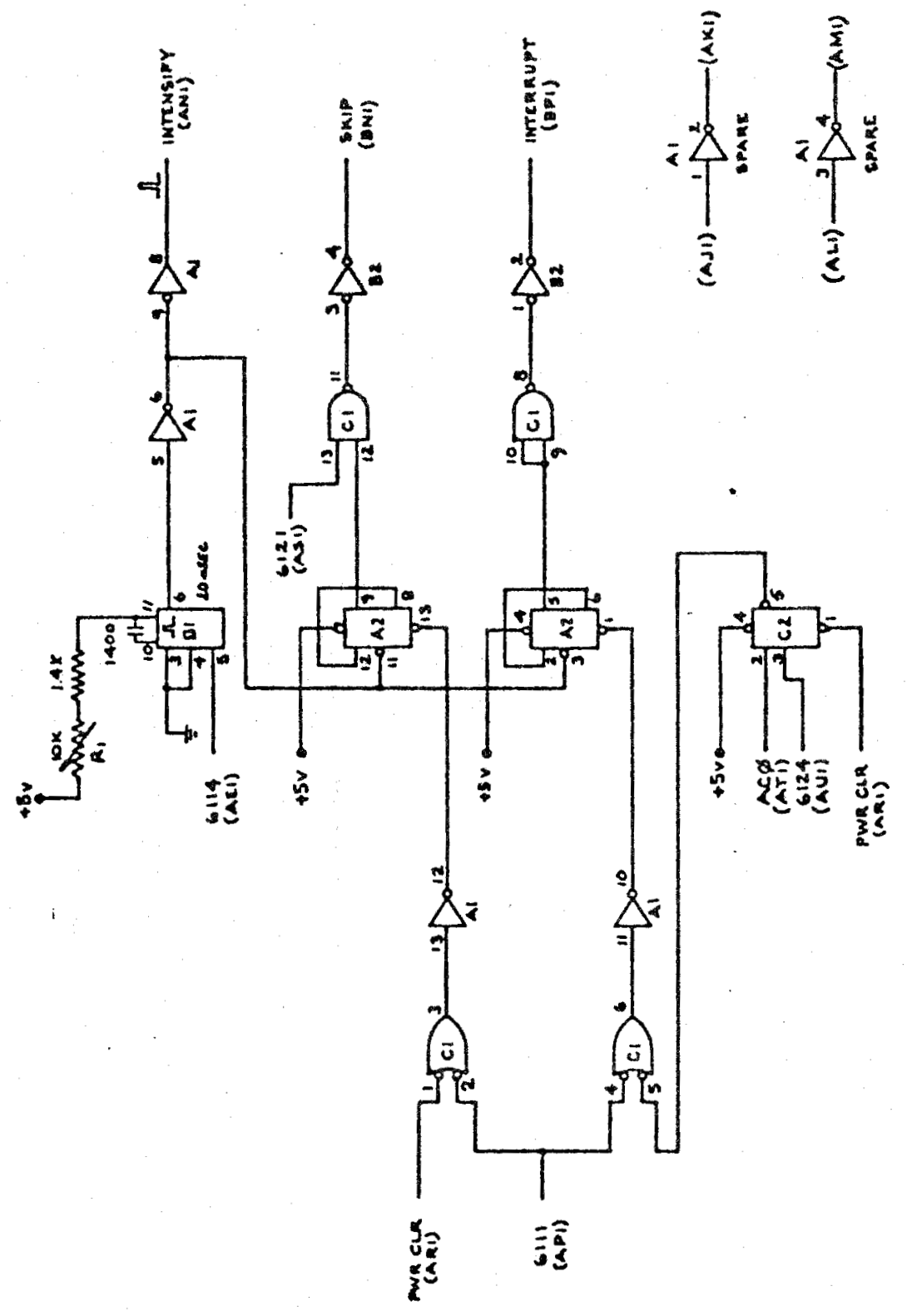

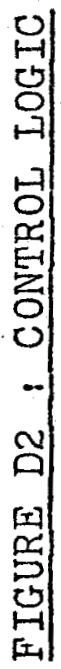




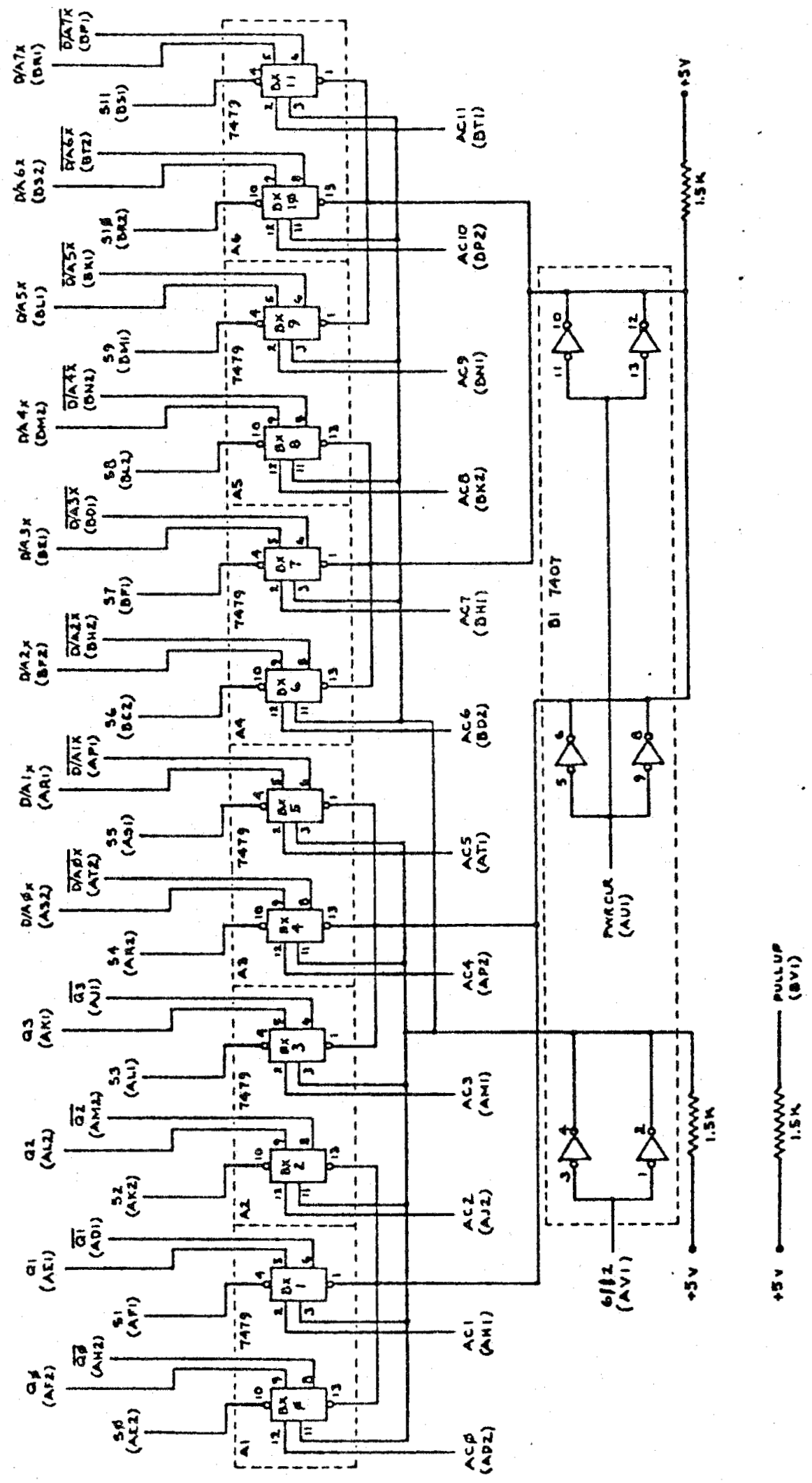

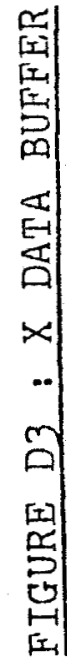




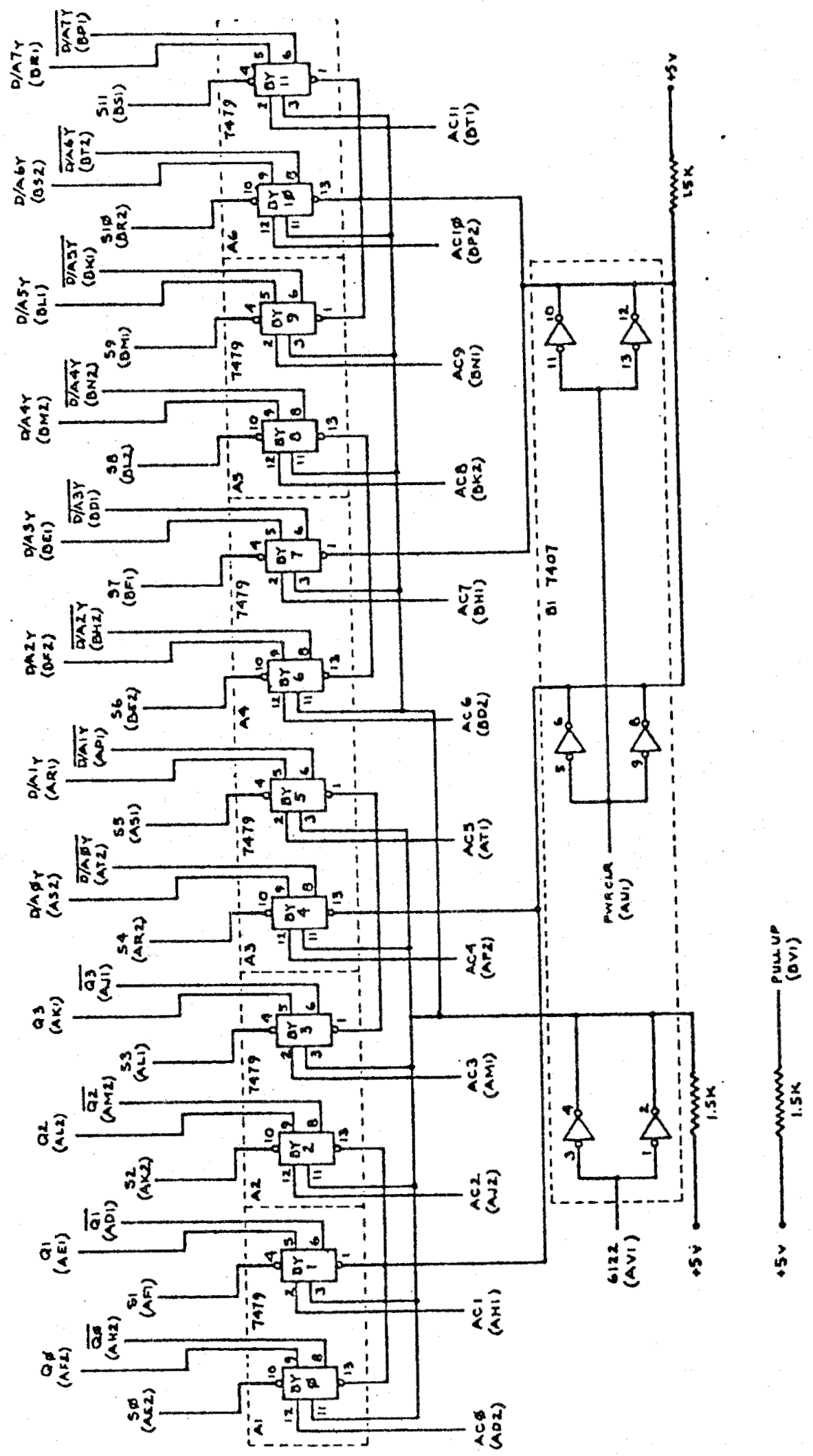



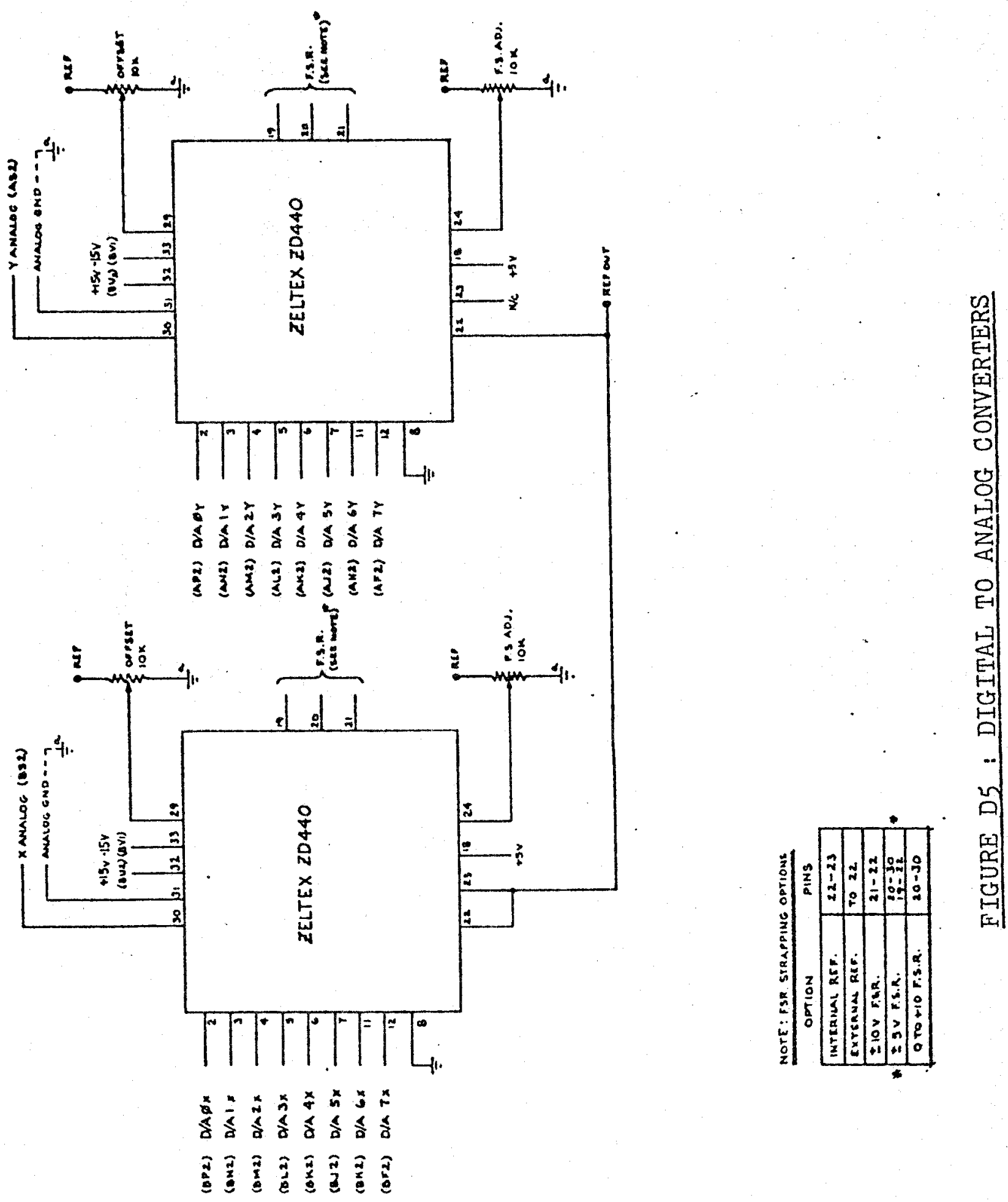
APPENDIX E

SCHEMATIC DIAGRAMS OF THE COMPLETE SYSTEM

Figures E1 and E2 show a general view of the CRT interface unit of the prototype unit. It consists of two units, $A$ and $B$. Unit $A$ consists of 8 circuit boards held in a single mounting rack via edge connector blocks. Interboard connections are formed by back panel wire wrapping. The rack also contains a Bluyne 5SP 3000 five volt, three ampere power supply and a Semiconductor Circuits Inc. Model No. LCD2,15,150 power supply, making the interface independent of other system power sources. The eight circuit boards and their functions are as follows:

1-5 Character generation

6 Device selectors and width buffer

$7 \mathrm{X}$ data buffer and $\mathrm{D} / \mathrm{A}$ converter

$8 Y$ data buffer and $D / A$ converter

Unit B consists of the circuits of line generation and intensity control. In order to eliminate the coupling of the $z$ pulse between the intensity control unit and the line generation unit, the $Z$ pulse amplifier of unit $B$ was isolated inside an enclosed steel box.

The schematic diagrams of the character generation unit are given in Appendix D. Since the function of all the circuits has been explained in the main thesis and since most of the circuits employed are standard and simple, 

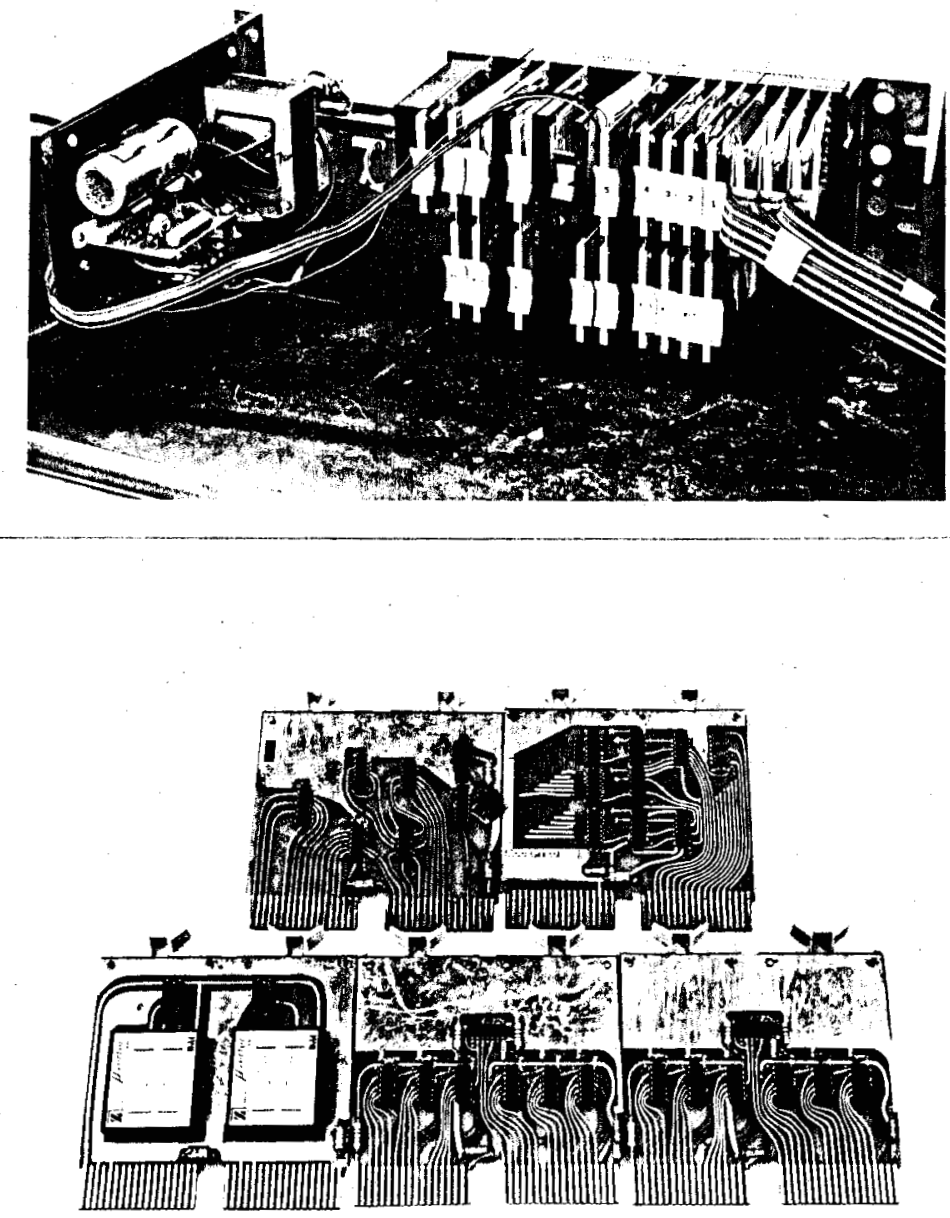

A GENERAL VIEW

OF THE UNIT A

\section{BOARD 1-5}
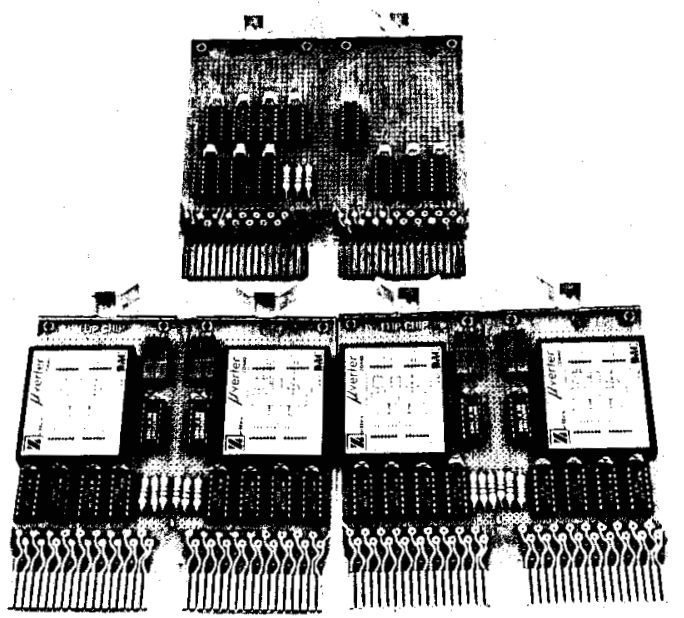

BOARD 6-8 


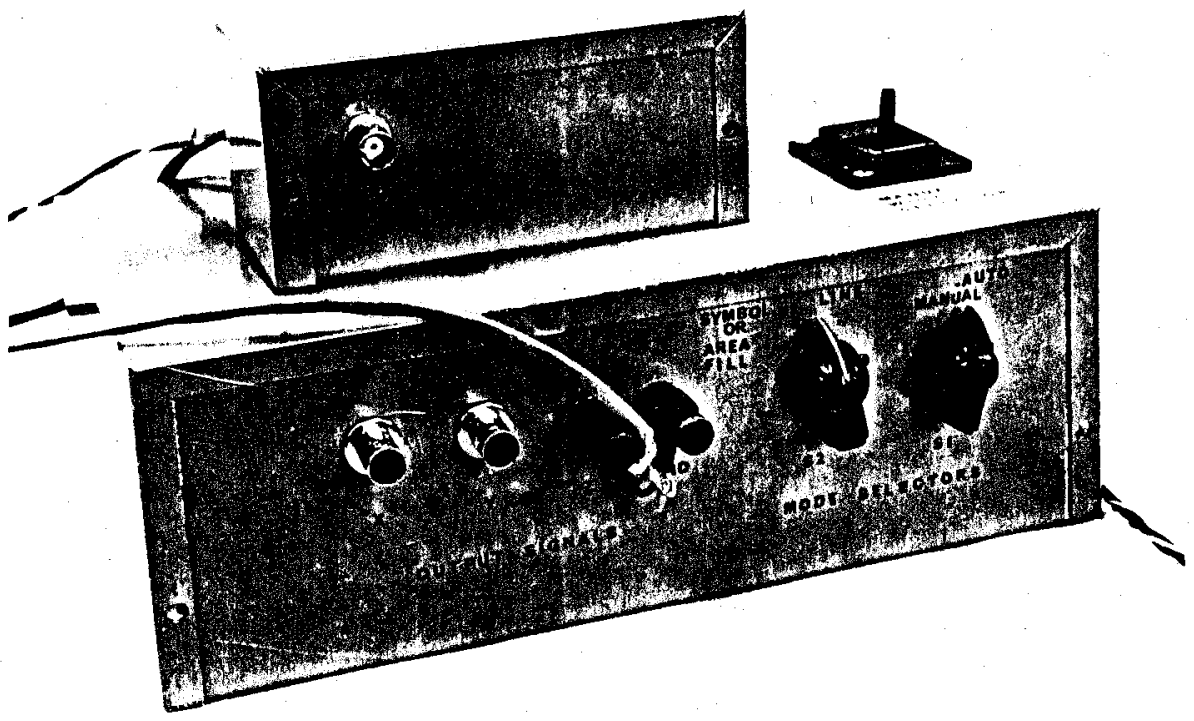

OUTSIDE VIEW OF THE UNIT B

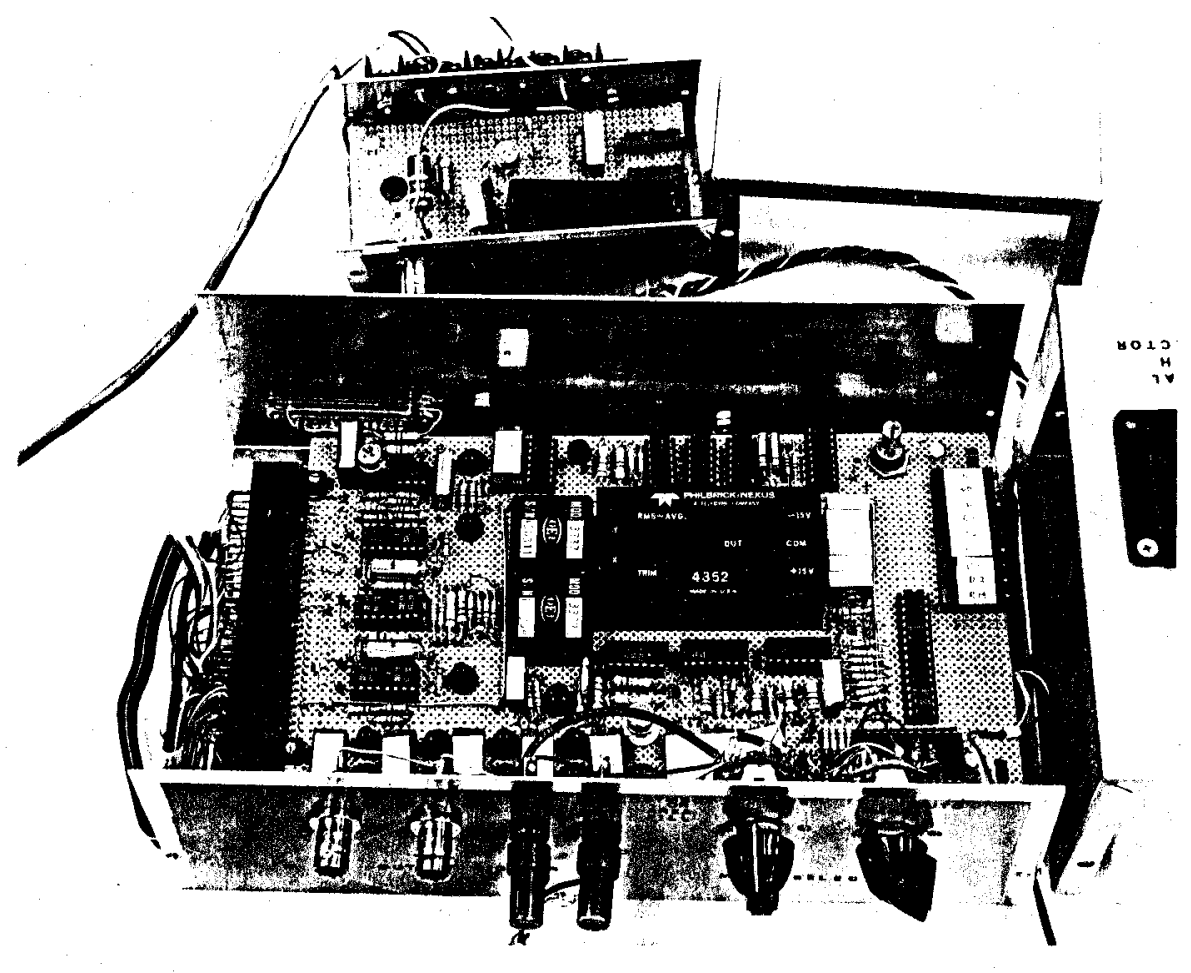

INSIDE VIEW OF THE UNIT B

FIGURE E2 UNIT B 
further explanation of the circuit design is not given. Figures E3 to E13 only show the schematic diagrams of the system $(18-31)$.

\section{PART LIST OF THE SYSTEM}

According to the schematic diagrams (Fig. E3 to Fig. E12), the components of the system are listed as follows:

\begin{tabular}{|c|c|}
\hline $\mathrm{COM}$ & {$[\mathrm{ON}$} \\
\hline 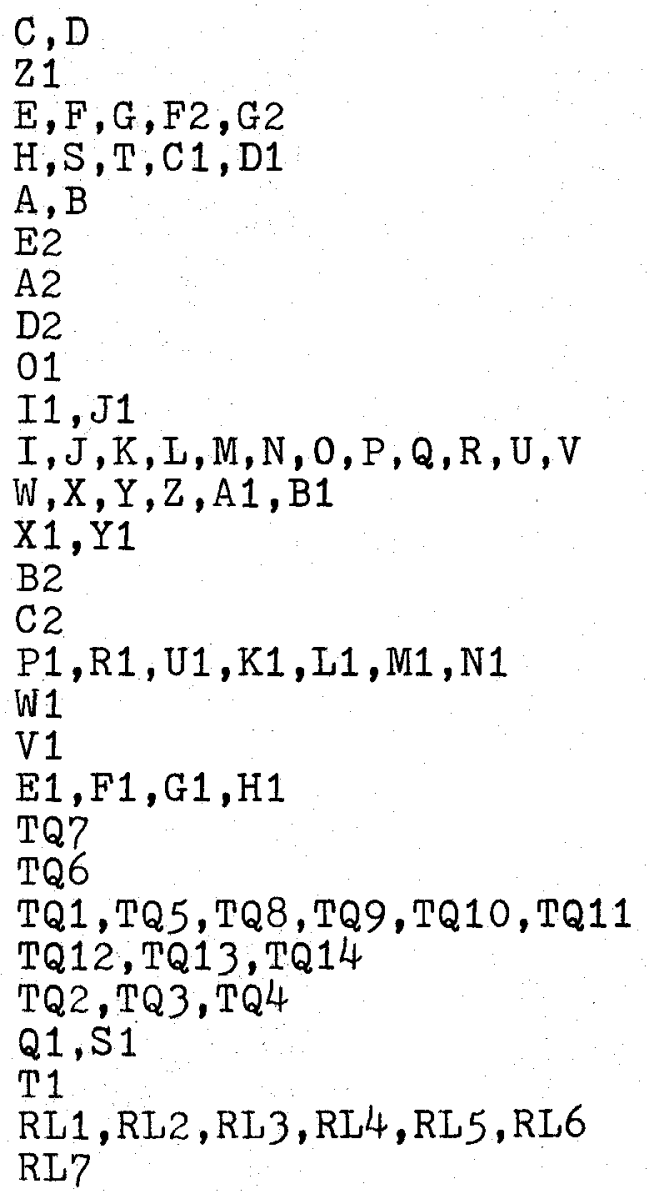 & $\begin{array}{l}\text { SN7400 } \\
\text { SN7402 } \\
\text { SN7404 } \\
\text { SN7407 } \\
\text { SN7430 } \\
\text { SN7440 } \\
\text { SN74121 } \\
\text { SN74154 } \\
\text { MC1439 } \\
\text { ML1458 } \\
\text { MC7479 } \\
\text { MC } 4779 \\
\text { MC8601 } \\
\text { LM301 } \\
\text { LN711 } \\
\text { Ma748 } \\
\text { NE566 } \\
\text { CD } 4046 \\
\text { ZD440 } \\
\text { MPS I01 } \\
\text { 2N4036 } \\
\text { 2N3638 } \\
\text { 2N3638 } \\
\text { 2N5163 } \\
\text { OPTICAL ELECTRONICS } 3337 \\
\text { PHILIBRICK } 4352 \\
\text { ELEC-TROL RA30222051 } \\
\text { ELEC-TROI RA30222051 }\end{array}$ \\
\hline
\end{tabular}




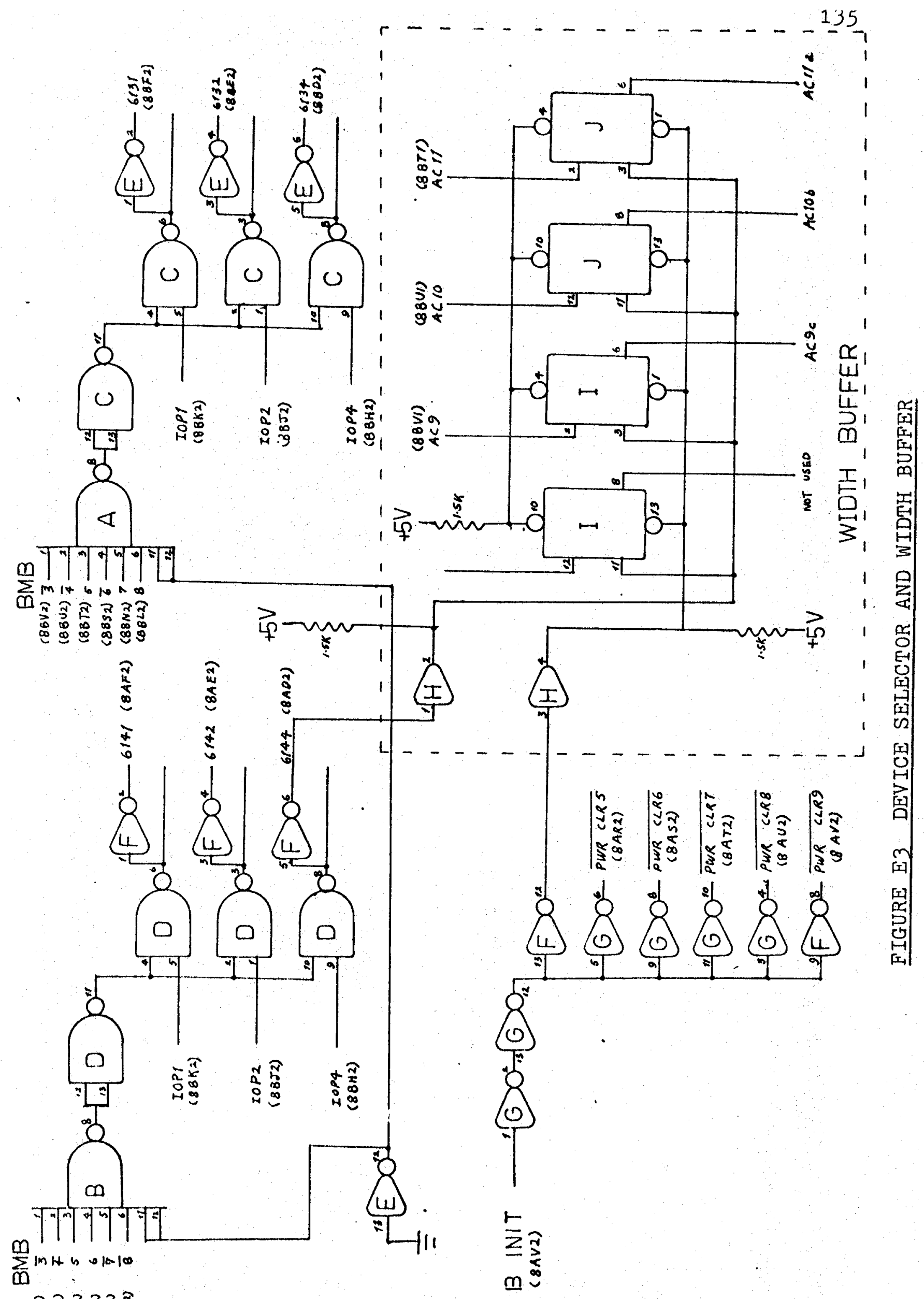

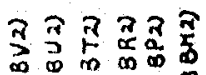

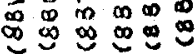




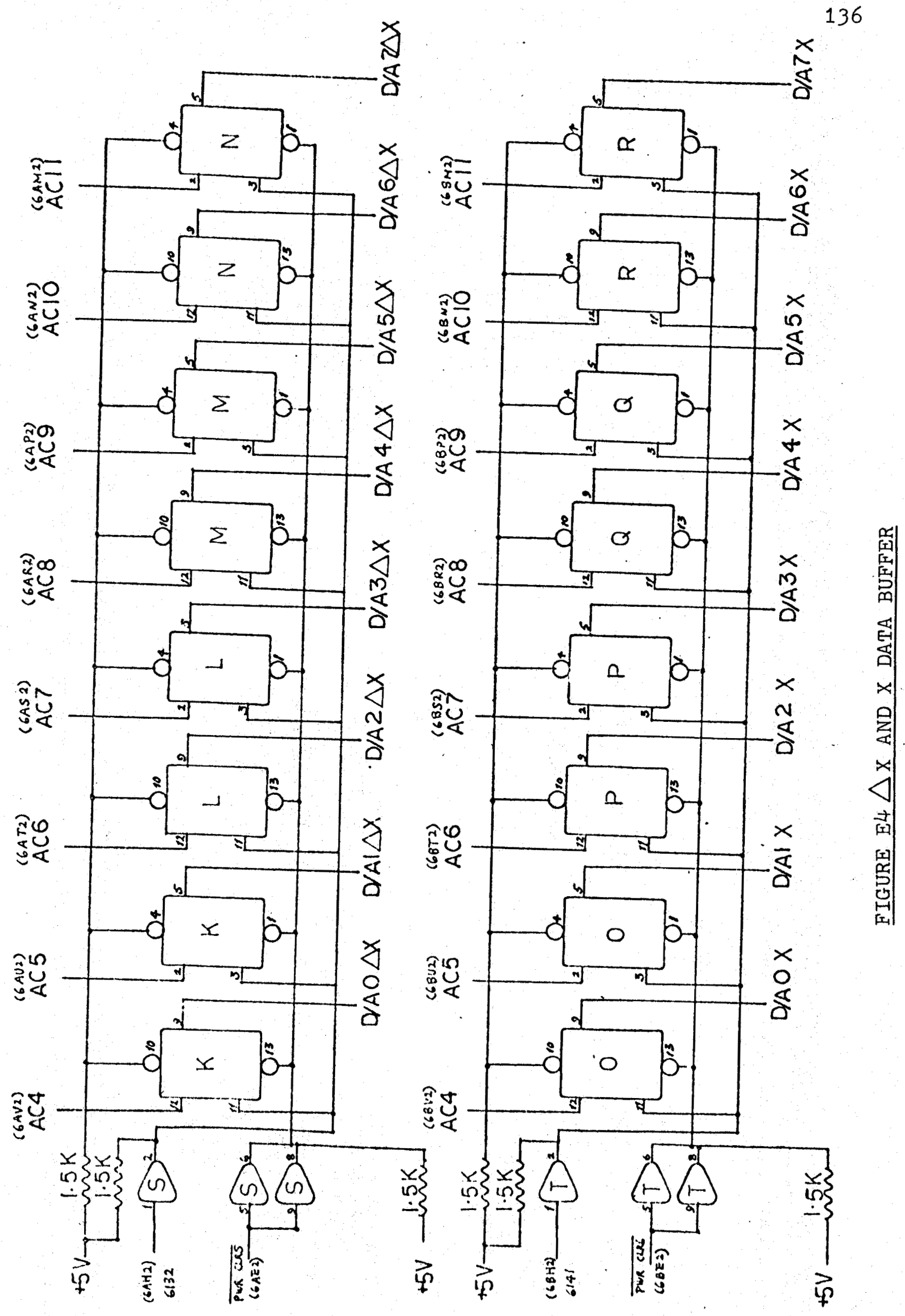




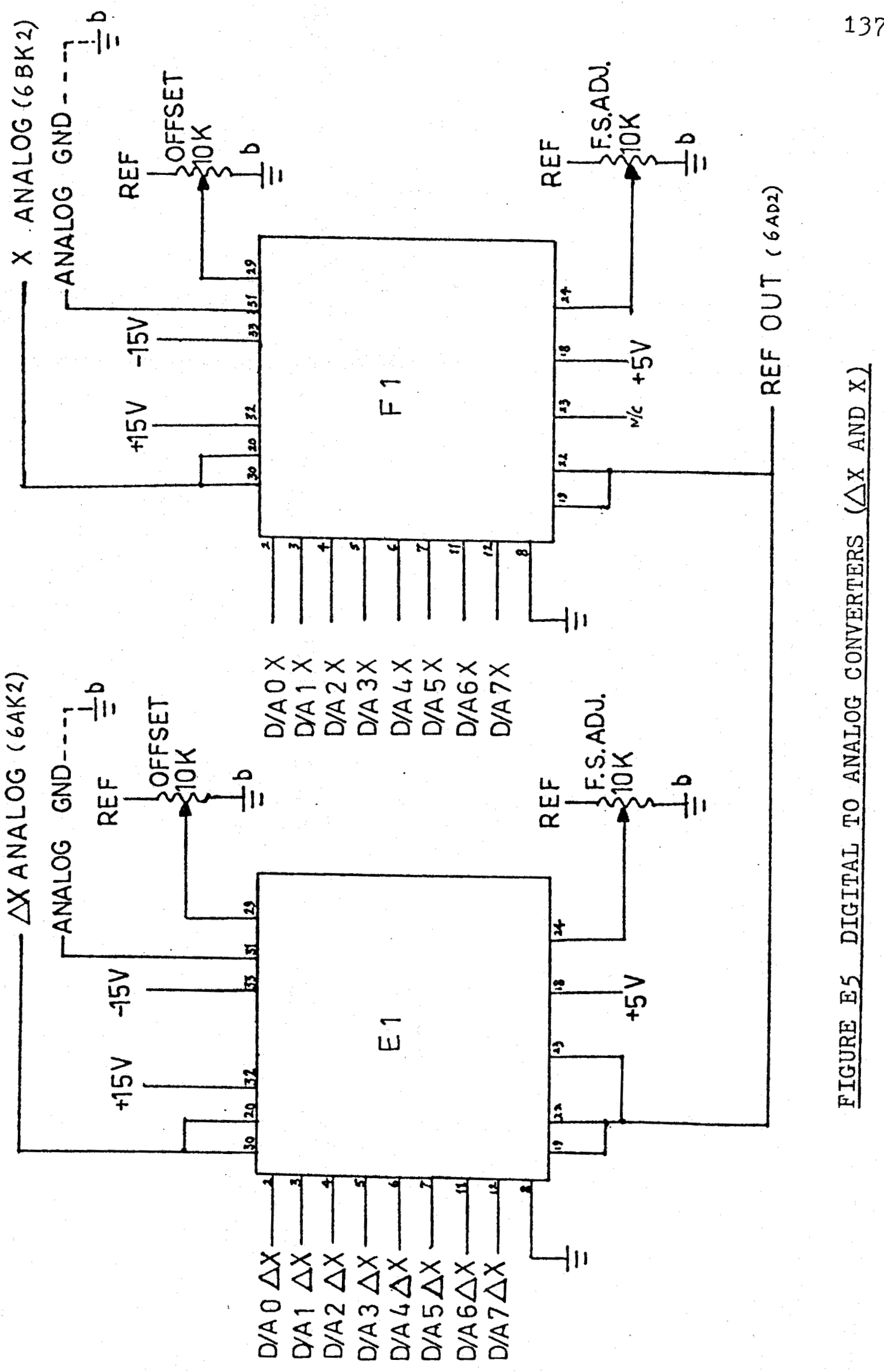




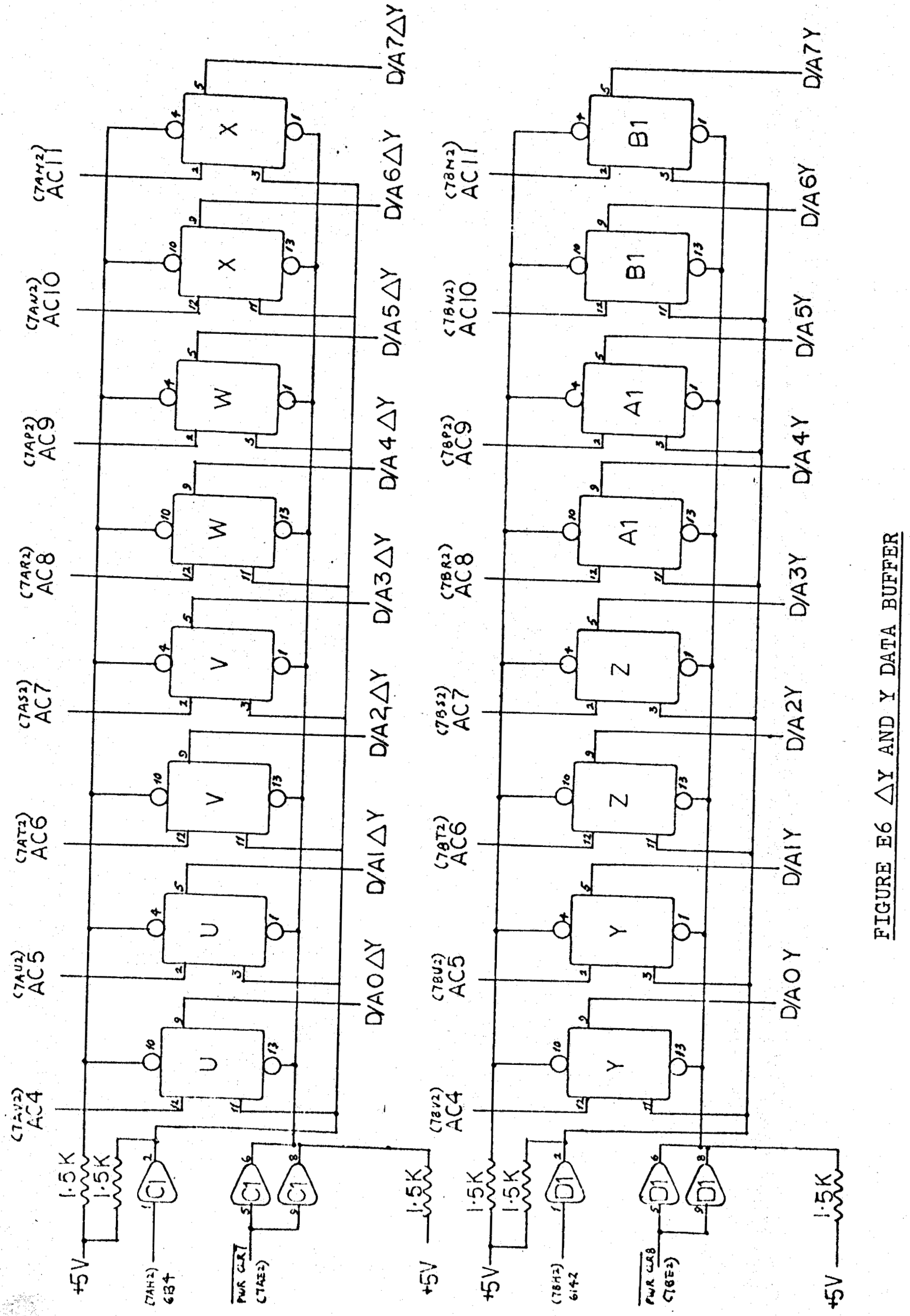




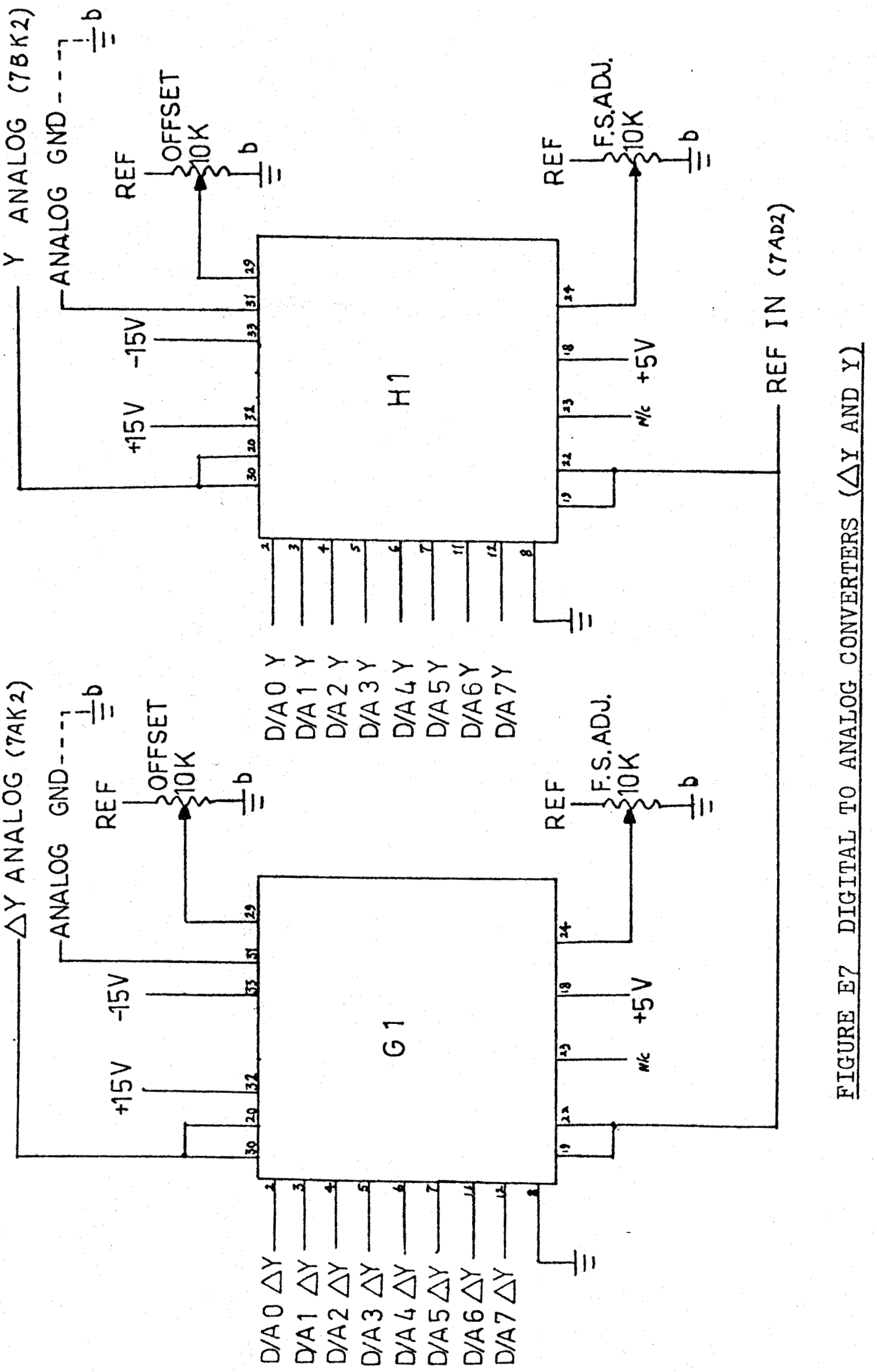




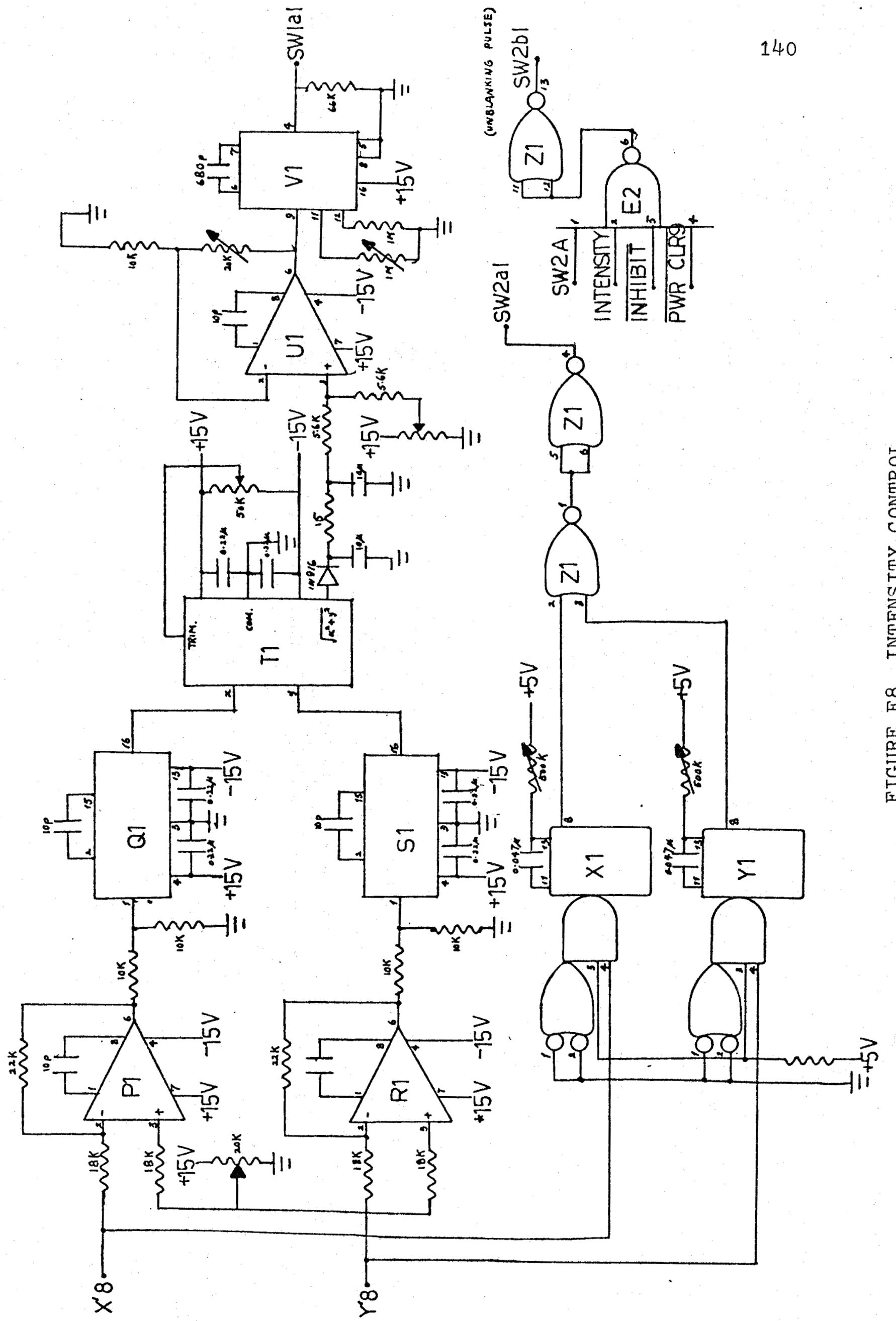




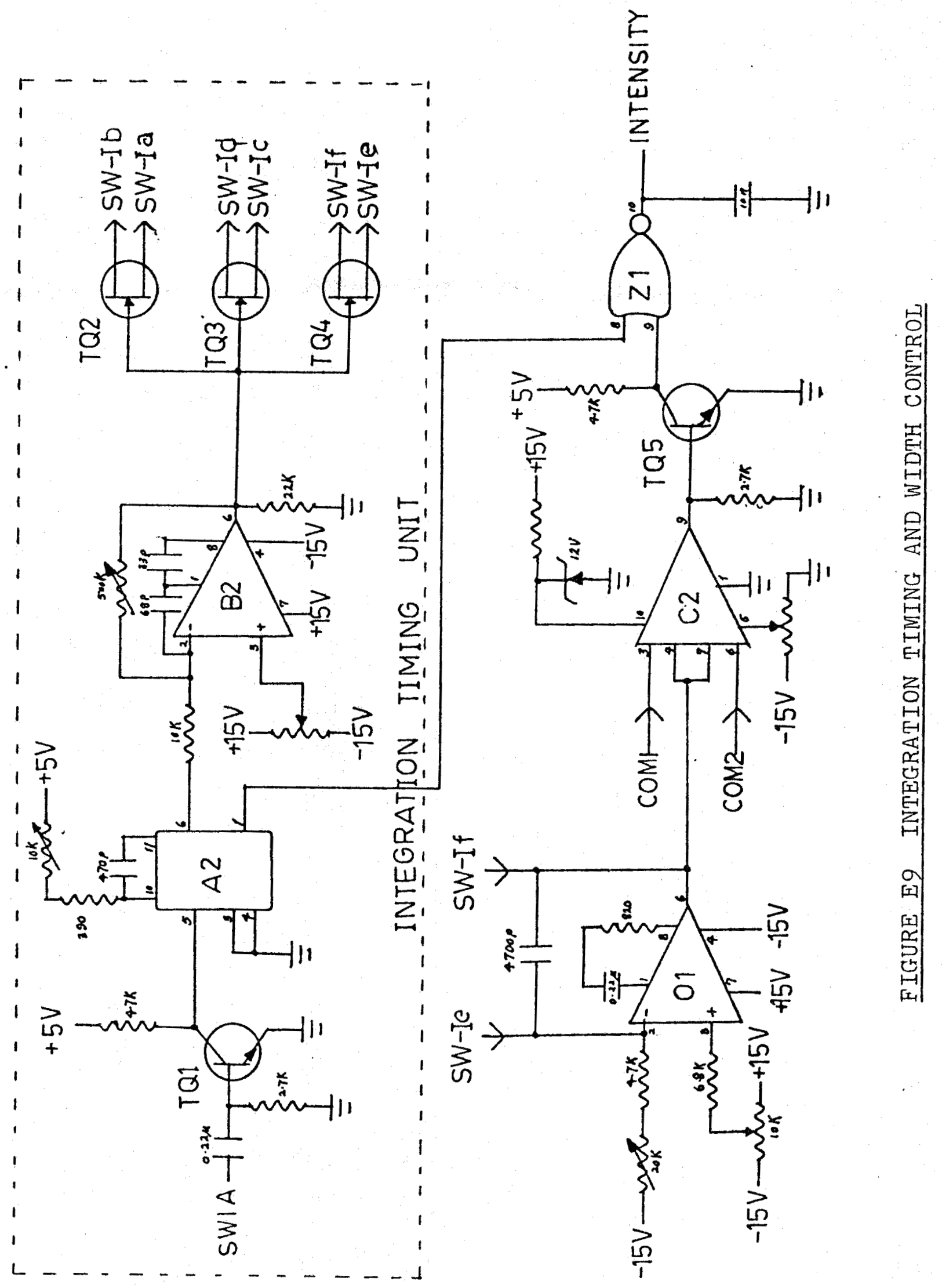




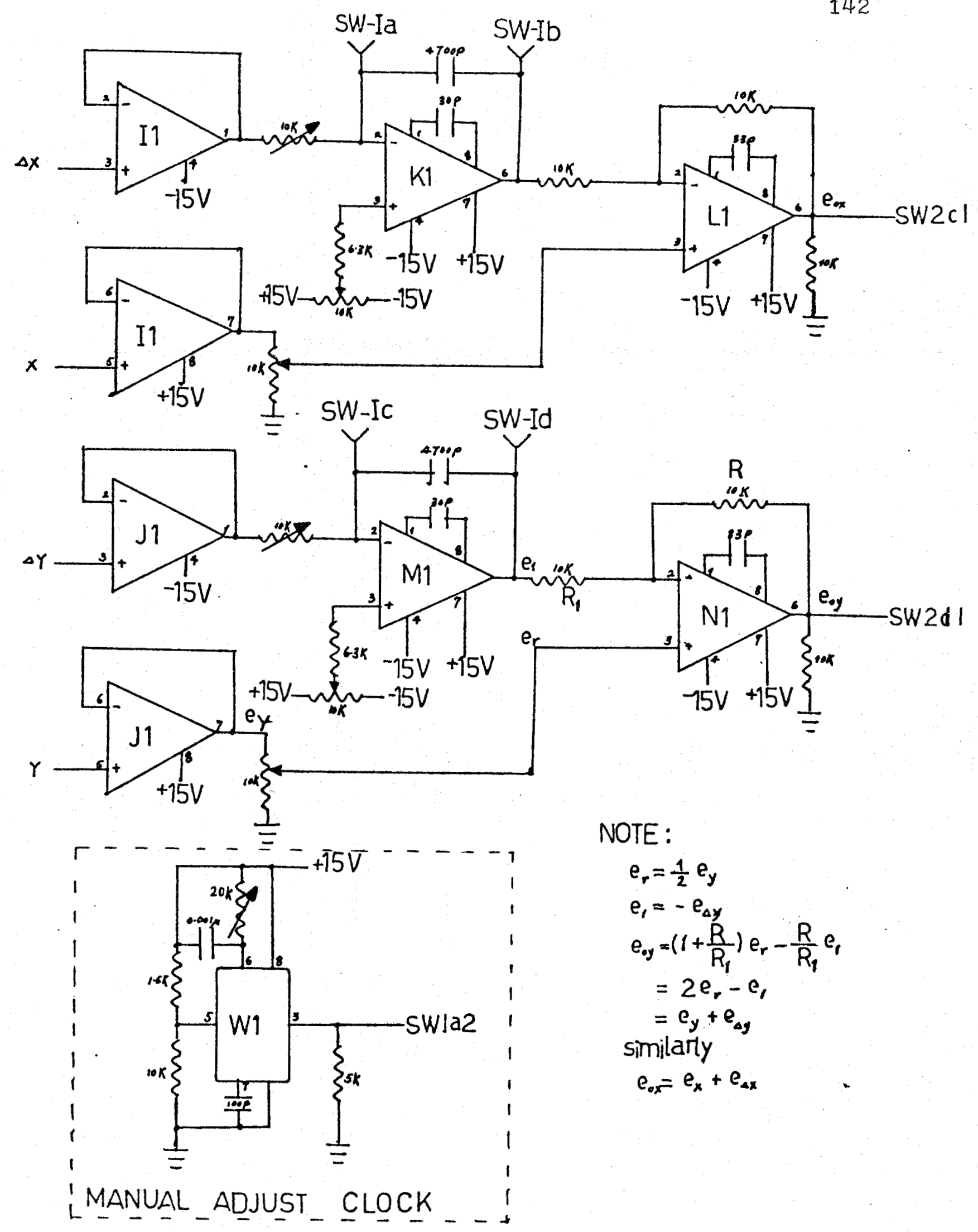

FIGURE E10 INTEGRATION AND SUMMING AMPLIFIERS AND MANUAL ADJUST CLOCK 


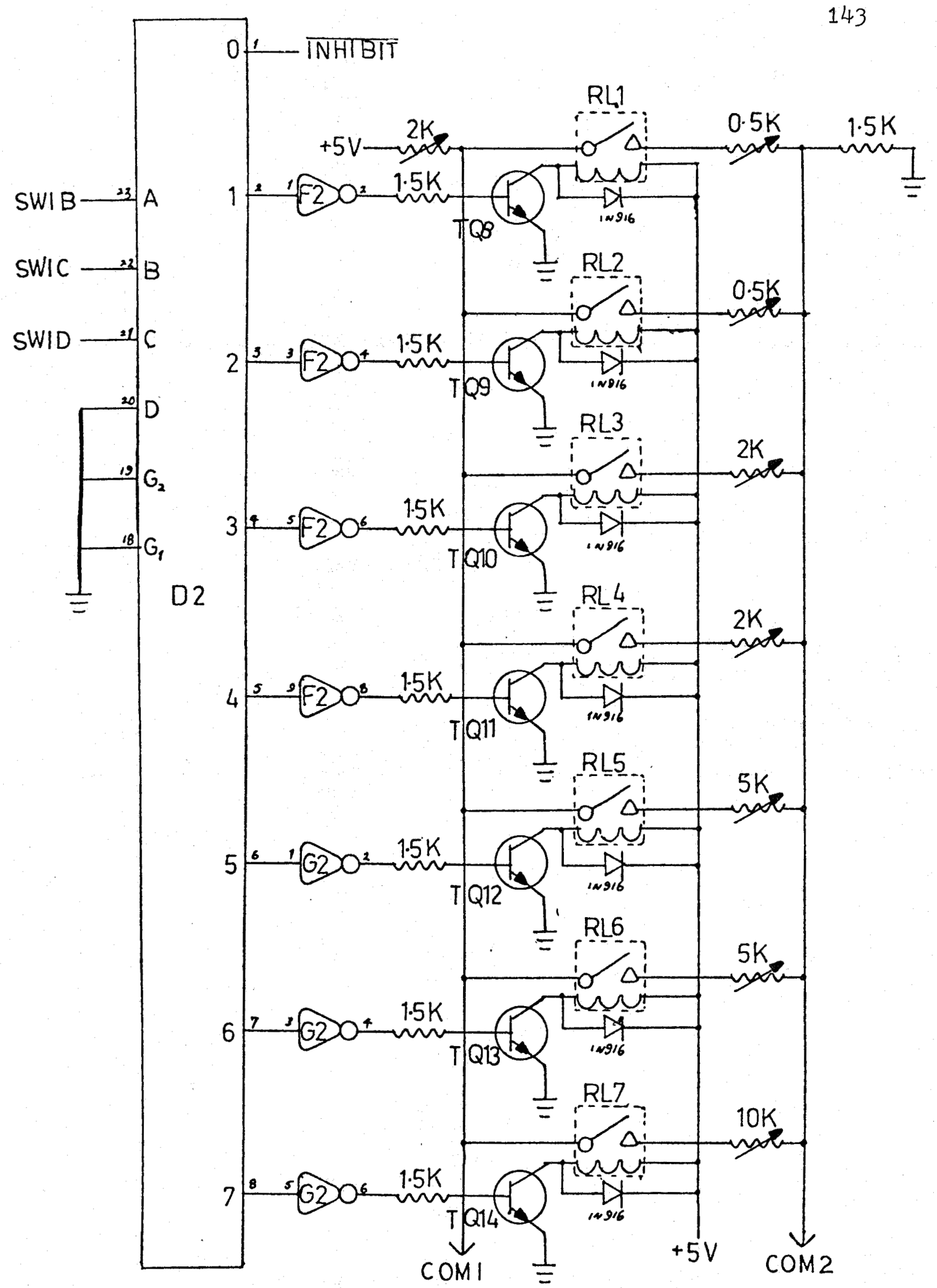

FIGURE E11 PROGRAMMABLE VOLTAGE DIVIDER 

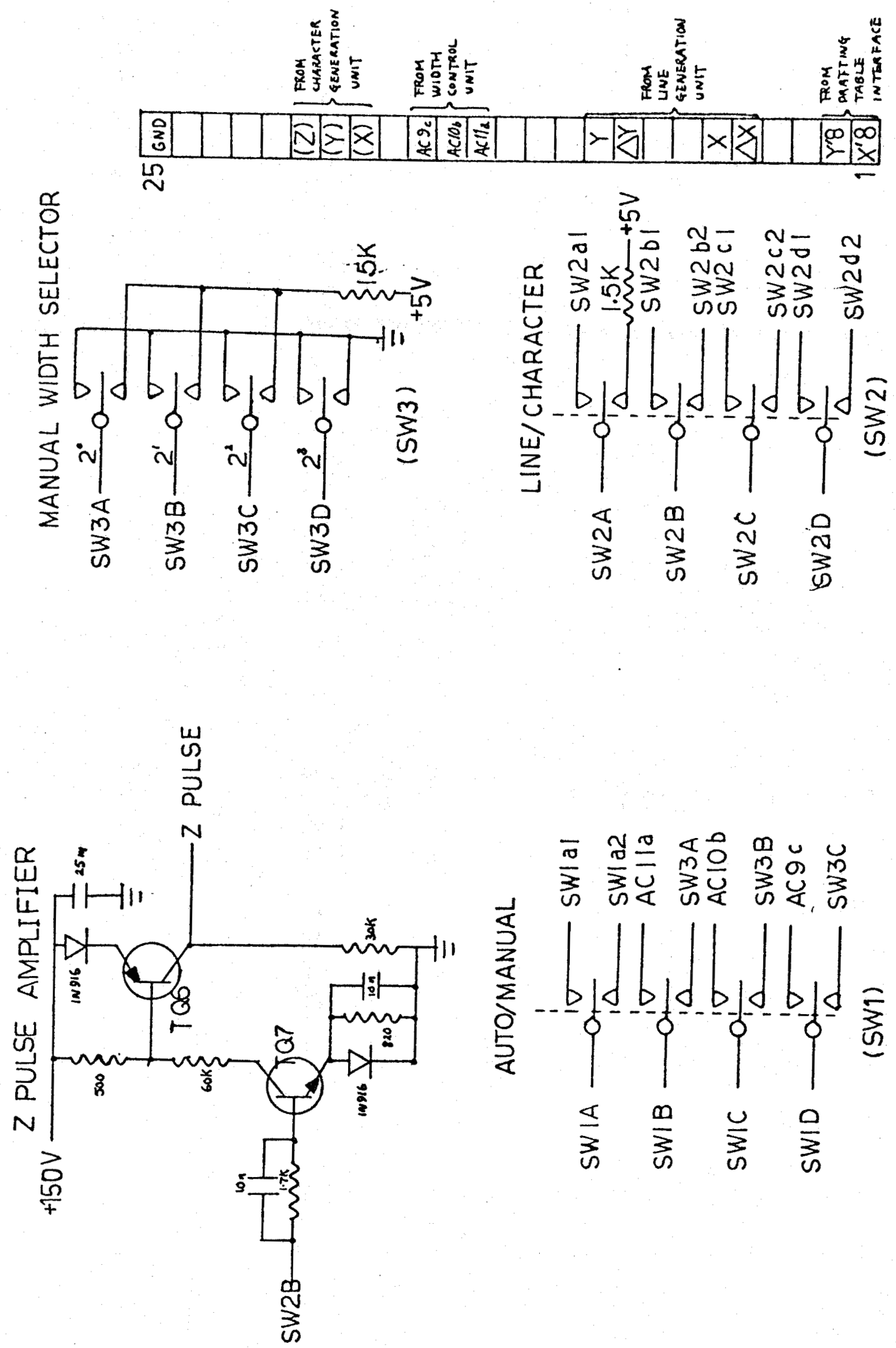


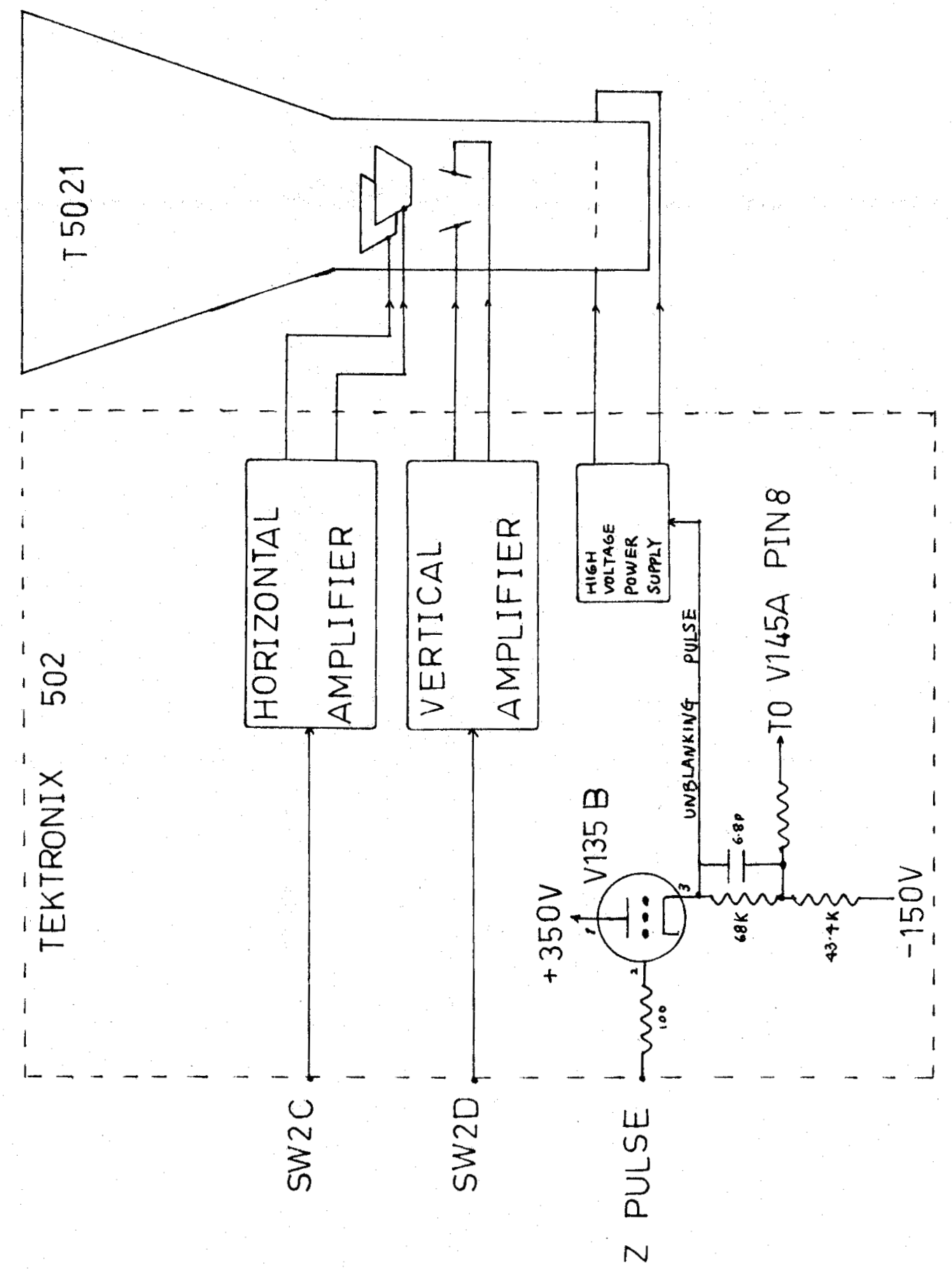

